\title{
Regularity and dimension spectrum of the equivariant spectral triple for the odd-dimensional quantum spheres
}

\author{
Arupkumar Pal and S. Sundar
}

\begin{abstract}
The odd-dimensional quantum sphere $S_{q}^{2 \ell+1}$ is a homogeneous space for the quantum group $\mathrm{SU}_{q}(\ell+1)$. A generic equivariant spectral triple for $S_{q}^{2 \ell+1}$ on its $L_{2}$ space was constructed by Chakraborty and Pal in [4]. We prove regularity for that spectral triple here. We also compute its dimension spectrum and show that it is simple. We give a detailed construction of its smooth function algebra and some related algebras that help proving regularity and in the computation of the dimension spectrum. Following the idea of Connes for $\mathrm{SU}_{q}(2)$, we first study another spectral triple for $S_{q}^{2 \ell+1}$ equivariant under torus group action and constructed by Chakraborty and Pal in [3]. We then derive the results for the $\mathrm{SU}_{q}(\ell+1)$-equivariant triple in the case $q=0$ from those for the torus equivariant triple. For the case $q \neq 0$, we deduce regularity and dimension spectrum from the case $q=0$.
\end{abstract}

Mathematics Subject Classification (2010). 58B34, 46L87, 19K33.

Keywords. Spectral triples, noncommutative geometry, quantum group.

\section{Contents}

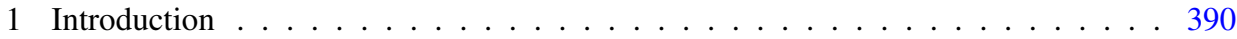

2 Preliminaries . . . . . . . . . . . . . . . . . . . . 392

2.1 Regular spectral triples . . . . . . . . . . . . . . . . . . . . . . . 392

2.2 Topological tensor products . . . . . . . . . . . . . . . . . . . . 393

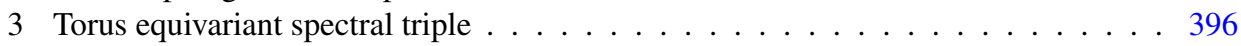

3.1 The spectral triple . . . . . . . . . . . . . . . . . . 396

3.2 The smooth function algebra $\mathscr{A}_{\ell}^{\infty} \ldots \ldots \ldots \ldots$. . . . . . . . . . . . . . . . . . . . . . . . . 497

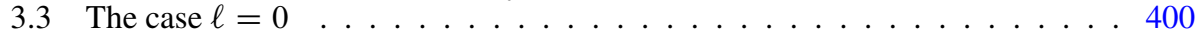

3.4 Regularity and the dimension spectrum . . . . . . . . . . . . . . . . 401

$4 \mathrm{SU}_{q}(\ell+1)$-equivariant spectral triple . . . . . . . . . . . . . . 405

4.1 Left multiplication operators . . . . . . . . . . . . . . . . . 405

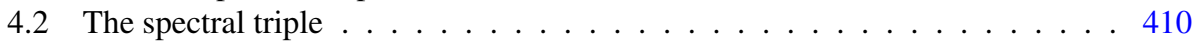

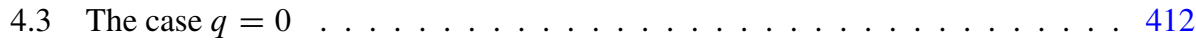

4.4 Regularity and dimension spectrum for $q \neq 0 \ldots \ldots \ldots \ldots$. . . . . . . . . . . . . . . 418

4.5 The smooth function algebra $C^{\infty}\left(S_{q}^{2 \ell+1}\right) \ldots \ldots \ldots \ldots . \ldots \ldots 420$

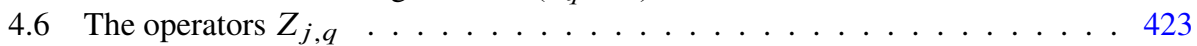

References . . . . . . . . . . . . . . . . . . 438 


\section{Introduction}

In noncommutative geometry, the starting point is usually a separable unital $\mathrm{C}^{*}$ algebra $A$ which is the noncommutative version of a compact Hausdorff space. Associated to this, one has certain invariants like the K-groups and the K-homology groups. In geometry, what one does next is to equip the topological space with a smooth structure so that in particular one can then talk about its de Rham cohomology. In the noncommutative situation, the parallel is to look for an appropriate dense subalgebra of $A$ that will play the role of smooth functions on the space. Given this dense subalgebra, one can compute various cohomology groups associated with it, namely the Hochschild cohomology, cyclic cohomology and the periodic cyclic cohomology, which are noncommutative and far-reaching generalizations of ordinary de Rham homology and cohomology. The question is: how to get hold of this dense subalgebra? One answer to this lies in the notion of a spectral triple, which plays a central role in Connes' formulation of noncommutative geometry.

In ordinary differential geometry, with just a smooth structure on a manifold, one can hardly go very far. In order that one can talk about shapes and sizes of spaces, one needs to bring in extra structure. One example is the Riemannian structure, which gives rise to a Riemannian connection, which in turn enables one to talk about curvature and so on. Other examples of such extra structures are Spin and $\mathrm{Spin}^{c}$ structures. In the presence of these extra structures, one has an operator-theoretic data that completely encodes the geometry. In noncommutative geometry, one takes this operatortheoretic data as the initial data and this is what goes by the name spectral triple.

Definition 1.1. Let $\mathcal{A}$ be an associative unital $*$-algebra. An even spectral triple for $\mathcal{A}$ is a triple $(\mathscr{H}, \pi, D)$ together with a $\mathbb{Z}_{2}$-grading $\gamma$ on $\mathscr{H}$ such that

(1) $\mathscr{H}$ is a (complex separable) Hilbert space,

(2) $\pi: \mathcal{A} \rightarrow \mathscr{L}(\mathscr{H})$ is a ${ }^{*}$-representation (usually assumed faithful),

(3) $D$ is a self-adjoint operator with compact resolvent such that $[D, \pi(a)] \in \mathscr{L}(\mathscr{H})$ for all $a \in \mathcal{A}$,

(4) $\pi(a) \gamma=\gamma \pi(a)$ for all $a \in \mathcal{A}$ and $D \gamma=-\gamma D$.

If no grading is present, one calls it an odd spectral triple.

The algebra $\mathcal{A}$ appearing in this definition is in general different from the $\mathrm{C}^{*}$ algebra $A$ one starts with. Typically it is a dense subalgebra in $A$, big enough so that the K-groups of $A$ and $\mathcal{A}$ coincide.

Since $D$ has compact resolvent, it has finite dimensional kernel. Hence by making a finite rank perturbation, one can make $D$ invertible. Now if one replaces $D$ with $\operatorname{sgn} D$, then one gets the notion of a Fredholm module over the algebra $\mathcal{A}$. If $\mathcal{A}$ is a dense *-subalgebra of a $\mathrm{C}^{*}$-algebra $A$, then this Fredholm module extends uniquely and gives a Fredholm module over $A$. In other words, one obtains an element in the K-homology group of $A$. This gives a map from the K-theory of $A$ to the set of 
integers via the K-theory-K-homology pairing. Starting from this Fredholm module, one can construct its Chern character, which gives an element in the periodic cyclic cohomology of $\mathcal{A}$, which, in turn, gives a map from the K-theory of $A$ to $\mathbb{C}$ via the periodic cyclic cohomology-K-theory pairing. The two maps thus obtained are the same. This is the content of the index theorem.

The Chern character is often difficult to compute. And that is where the spectral triple comes into the picture. Under certain hypothesis on the spectral triple, one can construct a cyclic cocycle, i.e., an element in the periodic cyclic cohomology that differs from the Chern character by a coboundary, so that it gives rise to the same map from $\mathrm{K}$-theory to $\mathbb{Z}$. Under a mild hypothesis on an invariant known as the dimension spectrum, these cocycles are given in terms of certain residue functionals, that can be relatively easier to compute. This is the Connes-Moscovici local index theorem ([7]), which is one of the major results in noncommutative geometry. It was first proved in the context of transverse geometry of foliations but is much more general in nature and has wider applicability. This was illustrated by Connes in [5], where he made a detailed analysis of the equivariant spectral triple for the quantum SU(2) group constructed in [2]. A similar analysis was later done by Dabrowski et al. in [14] for the spectral triple constructed in [8]. Typically, the $\mathrm{C}^{*}$-algebras associated to quantum groups or their homogeneous spaces are given by a set of generators and relations. While constructing spectral triples, one does it for the associated coordinate function algebra, i.e., the *-subalgebra generated by these generators. This algebra is not closed under the holomorphic function calculus of the $\mathrm{C}^{*}$-algebra. Therefore one needs to construct the smooth function algebra, to prove regularity and to compute the dimension spectrum in order to be able to apply the Connes-Moscovici theorem. This was done in [5], where Connes also defined a symbol map and gave formulae for computing the residue functionals in terms of the symbol maps.

Odd-dimensional quantum spheres are higher-dimensional analogues of the quantum $\mathrm{SU}(2)$. The $(2 \ell+1)$ dimensional sphere $S_{q}^{2 \ell+1}$ is a homogeneous space of the quantum group $\mathrm{SU}_{q}(\ell+1)$. In [4], Chakraborty and Pal constructed a generic spectral triple on the $L_{2}$-space of the sphere with non-trivial K-homology class and equivariant under the action of $\mathrm{SU}_{q}(\ell+1)$. The main aim of the present article is to prove that this spectral triple is regular. We also introduce the smooth function algebra and compute the dimension spectrum. The dimension spectrum is shown to be simple so that the Connes-Moscovici local index theorem is applicable to this triple. The local index computation will be taken up in a separate article.

Here is a brief outline of the contents of this article. In the next section, we recall a few basic notions from [7]. We then collect together a few observations and remarks on tensor products of Fréchet algebras and fix some of the notations. In Section 3, we first look at the torus equivariant spectral triple for the spheres and introduce a smooth function algebra, prove regularity and compute the dimension spectrum. The key here is the short exact sequence

$$
0 \longrightarrow \mathcal{K} \otimes C(\mathbb{T}) \longrightarrow C\left(S_{q}^{2 \ell+1}\right) \longrightarrow C\left(S_{q}^{2 \ell-1}\right) \longrightarrow 0
$$


and results by Schweitzer ([12], [13]) on spectral invariance. Using these and the idea employed by Connes in [5], we build the smooth function algebra over $S_{q}^{2 \ell+1}$ recursively starting from $C^{\infty}(\mathbb{T})$.

In Section 4, we deal with the $\mathrm{SU}_{q}(\ell+1)$-equivariant spectral triple. We first treat the case $q=0$. Using a decomposition of the $L_{2}$-space, we relate it to the torus equivariant triple with a certain multiplicity. Regularity, smooth function algebra and properties of the dimension spectrum all then follow from the results in Section 3. Here again the idea is exactly as in [5] for $\mathrm{SU}_{q}(2)$. In Section 4.4 we treat the case $q \neq 0$. We take a close look at the representation of the algebra, in particular the images of the generating elements, and after a careful analysis we prove that, modulo operators that one can neglect for the purpose of computing the dimension spectrum, things can be deduced from the torus equivariant case again.

\section{Preliminaries}

2.1. Regular spectral triples. In this section we recall some definitions and notions from [7]. Let $D$ be a selfadjoint operator on a Hilbert space $\mathscr{H}$ which is invertible. Define $\mathscr{H}_{s}=\operatorname{Dom}\left(|D|^{s}\right)$ for $s \geq 0$. Then $\mathscr{H}_{s}$ is a decreasing family of vector

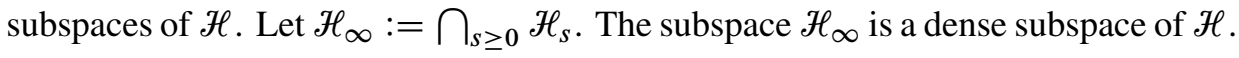

Definition 2.1. An operator $T: \mathscr{H}_{\infty} \rightarrow \mathscr{H}_{\infty}$ is said to be smoothing if for every $m, n \geq 0$ the operator $|D|^{m} T|D|^{n}$ is bounded. The vector space of smoothing operators is denoted by $\mathrm{OP}^{-\infty}$.

For $T \in \mathrm{OP}^{-\infty}$, define $\|T\|_{m, n}=\left\||D|^{m} T|D|^{n}\right\|$ for $m, n \geq 0$.

Lemma 2.2. The vector space $\mathrm{OP}^{-\infty}$ is an involutive subalgebra of $\mathscr{L}(\mathscr{H})$ and equipped with the family of seminorms $\|\cdot\|_{m, n}$ is a Fréchet algebra.

Let $\delta$ be the unbounded derivation $[|D|, \cdot]$. More precisely, $\operatorname{Dom}(\delta)$ consists of all bounded operators $T$ that leave $\operatorname{Dom}(|D|)$ invariant and for which $\delta(T):=[|D|, T]$ extends to a bounded operator.

Lemma 2.3 ([6]). The unbounded derivation $\delta$ is a closed derivation, i.e., if $T_{n}$ is a sequence in $\operatorname{Dom}(\delta)$ such that $T_{n} \rightarrow T$ and $\delta\left(T_{n}\right) \rightarrow S$ then $T \in \operatorname{Dom}(\delta)$ and $\delta(T)=S$.

Define $\mathrm{OP}^{0}:=\left\{T \in \mathscr{L}(\mathscr{H}): T \in \bigcap_{n} \operatorname{Dom}\left(\delta^{n}\right)\right\}$. The following lemma says that elements of $\mathrm{OP}^{0}$ are operators on $\mathscr{H}_{\infty}$.

Lemma 2.4. Let $T$ be a bounded operator on $\mathcal{H}$. Then the following are equivalent.

(1) The operator $T \in \mathrm{OP}^{0}$. 
(2) The operator $T$ leaves $\mathscr{H}_{\infty}$ invariant and $\delta^{n}(T): \mathscr{H}_{\infty} \rightarrow \mathscr{H}_{\infty}$ is bounded for every $n \in \mathbb{N}$.

It is easy to see from Lemma 2.4 that $\mathrm{OP}^{0}$ is an algebra and that $|D|^{-m} T|D|^{m}$ is bounded for every $m \in \mathbb{Z}$ if $T \in \mathrm{OP}^{0}$. As a consequence it follows that $\mathrm{OP}^{-\infty}$ is an ideal in $\mathrm{OP}^{0}$. Now we recall the notions of regularity and dimension spectrum for a spectral triple.

Definition 2.5. Let $(\mathcal{A}, \mathscr{H}, D)$ be a spectral triple. We say that $(\mathcal{A}, \mathscr{H}, D)$ is regular if $\mathcal{A}+[D, \mathcal{A}] \subset \mathrm{OP}^{0}$.

A spectral triple $(\mathcal{A}, \mathscr{H}, D)$ is $p+$ summable if $|D|^{-p}$ is in the ideal of Dixmier traceable operators $\mathscr{L}^{(1, \infty)}$. In particular, if $(\mathcal{A}, \mathscr{H}, D)$ is $p+$ summable, then $|D|^{-s}$ is trace class for $s>p$. Let $(\mathcal{A}, \mathcal{H}, D)$ be a regular spectral triple which is $p+$ summable for some $p$. Let $\mathscr{B}$ be the algebra generated by $\delta^{n}(\mathcal{A})$ and $\delta^{n}([D, \mathcal{A}])$. We say that the spectral triple $(\mathcal{A}, \mathscr{H}, D)$ has discrete dimension spectrum $\Sigma \subset \mathbb{C}$ if $\Sigma$ is discrete and, for every $b \in \mathscr{B}$, the function $\operatorname{Trace}\left(b|D|^{-z}\right)$ initially defined for $\operatorname{Re}(z)>p$ extends to a meromorphic function with poles only in $\Sigma$. We say the dimension spectrum is simple if all the poles are simple.

2.2. Topological tensor products. The $\mathrm{C}^{*}$-algebras involved in tensor products that we deal with in this paper are all nuclear. Therefore no ambiguities arise due to nonuniqueness of tensor products. Apart from $\mathrm{C}^{*}$-algebras and their tensor products, we will also deal with Fréchet algebras and their tensor products. Suppose that $A_{1}$ and $A_{2}$ are two Fréchet algebras with topologies coming from the families of seminorms $\left(\|\cdot\|_{\lambda}\right)_{\lambda \in \Lambda}$ and $\left(\|\cdot\|_{\lambda^{\prime}}\right)_{\lambda^{\prime} \in \Lambda^{\prime}}$. For each pair $\left(\lambda, \lambda^{\prime}\right) \in \Lambda \times \Lambda^{\prime}$, one forms the projective cross norm $\|\cdot\|_{\lambda, \lambda^{\prime}}$, which is a seminorm on the algebraic tensor product $A_{1} \otimes_{\text {alg }} A_{2}$. The family $\left(\|\cdot\|_{\lambda, \lambda^{\prime}}\right)_{\left(\lambda, \lambda^{\prime}\right) \in \Lambda \times \Lambda^{\prime}}$ then gives rise to a topology on $A_{1} \otimes_{\text {alg }} A_{2}$. Completion with respect to this is a Fréchet algebra and is called the projective tensor product of $A_{1}$ and $A_{2}$. While talking about tensor product of two Fréchet algebras, we will always mean their projective tensor product and will denote it by $A_{1} \otimes A_{2}$.

We will mainly be concerned with Fréchet algebras sitting inside some $\mathscr{L}(\mathscr{H})$ with Fréchet topology finer than the norm topology. In other words, we will be dealing with Fréchet algebras with faithful continuous representations on Hilbert spaces. Let $A_{1}, A_{2}$ be Fréchet algebras. If $\rho_{i}: A_{i} \rightarrow \mathscr{L}\left(\mathscr{H}_{i}\right)$ are continuous representations for $i=0,1$, where the $\mathscr{H}_{i}$ 's are Hilbert spaces, then by the universality of the projective tensor product it follows that there exists a unique continuous representation $\rho_{1} \otimes \rho_{2}: A_{1} \otimes A_{2} \rightarrow \mathscr{L}\left(\mathscr{H}_{1} \otimes \mathscr{H}_{2}\right)$ such that $\left(\rho_{1} \otimes \rho_{2}\right)\left(a_{1} \otimes a_{2}\right)=\rho_{1}\left(a_{1}\right) \otimes \rho_{2}\left(a_{2}\right)$. If the $A_{i}$ 's are subalgebras of $\mathscr{L}\left(\mathscr{H}_{i}\right)$ then we will call the tensor product representation of $A_{1} \otimes A_{2}$ on $\mathscr{H}_{1} \otimes \mathscr{H}_{2}$ the natural representation.

Lemma 2.6. Let $\left(A_{1}, \mathcal{H}_{1}, D_{1}\right)$ and $\left(A_{2}, \mathscr{H}_{2}, D_{2}\right)$ be regular spectral triples. Assume that the following conditions hold: 
(1) The algebras $A_{1}$ and $A_{2}$ are Fréchet algebras represented faithfully on $\mathscr{H}_{1}$ and $\mathscr{H}_{2}$, respectively.

(2) The selfadjoint operators $D_{1}$ and $D_{2}$ are positive with compact resolvent.

(3) For $i=0,1$, the unbounded derivations $\delta_{i}=\left[D_{i}, \cdot\right]$ leave $A_{i}$ invariant and $\delta_{i}: A_{i} \rightarrow A_{i}$ is continuous.

Let $D:=D_{1} \otimes 1+1 \otimes D_{2}$. Suppose that the natural representation of $A_{1} \otimes A_{2}$ on $\mathscr{H}_{1} \otimes \mathscr{H}_{2}$ is faithful. Then the triple $\left(A_{1} \otimes A_{2}, \mathscr{H}_{1} \otimes \mathscr{H}_{2}, D\right)$ is a regular spectral triple. More precisely, the unbounded derivation $\delta:=[D, \cdot]$ leaves the algebra $A_{1} \otimes A_{2}$ invariant and the map $\delta: A_{1} \otimes A_{2} \rightarrow A_{1} \otimes A_{2}$ is continuous.

Proof. Let $\delta^{\prime}=\delta_{1} \otimes 1+1 \otimes \delta_{2}$. Then $\delta^{\prime}$ is a continuous linear operator on $A_{1} \otimes A_{2}$. Clearly $A_{1} \otimes_{\text {alg }} A_{2} \subset \operatorname{Dom}(\delta)$ and $\delta=\delta^{\prime}$ on $A_{1} \otimes_{\text {alg }} A_{2}$. Now let $a \in A_{1} \otimes A_{2}$ be given. Choose a sequence $\left(a_{n}\right) \in A_{1} \otimes_{\text {alg }} A_{2}$ such that $a_{n} \rightarrow a$ in $A_{1} \otimes A_{2}$. Then $a_{n} \rightarrow a$ in $\mathscr{L}\left(\mathscr{H}_{1} \otimes \mathscr{H}_{2}\right)$. Since $\delta^{\prime}$ is continuous and because the inclusion $A_{1} \otimes A_{2} \subset \mathscr{L}\left(\mathscr{H}_{1} \otimes \mathscr{H}_{2}\right)$ is continuous, it follows that $\left(\delta^{\prime}\left(a_{n}\right)\right)=\left(\delta\left(a_{n}\right)\right)$ is a Cauchy sequence in $\mathscr{L}\left(\mathscr{H}_{1} \otimes \mathscr{H}_{2}\right)$. Since $\delta$ is closed, this implies that $a \in \operatorname{Dom}(\delta)$ and $\delta(a)=\delta^{\prime}(a)$. Now the lemma follows.

The above lemma can be extended to tensor product of finite number of spectral triples with the appropriate assumptions.

Remark 2.7. Note that the above lemma is not meant to apply to a very general set up. The assumptions that the $D_{i}$ 's are positive and the algebras $A_{i}$ are invariant under the derivations $\delta_{i}$ are rather unusual for a spectral triple. A spectral triple will normally not satisfy these requirements. For example, positivity of $D$ means that the spectral triple has trivial K-homology class, giving trivial pairing with K-theory. Also, the operator $D$ is not given by the usual product formula for two Dirac operators. We will use this lemma in Section 4 in a very specific situation, where $D_{i}$ is $\left|\widetilde{D}_{i}\right|$ for Dirac operators $\widetilde{D}_{i}$ and $D$ is the absolute value of the Dirac operator for the product space that we are interested in. Also the $A_{i}$ 's are of very specific nature so that invariance under $\delta_{i}$ is satisfied. The main purpose of the lemma is to derive, in our situation, the invariance of $A_{1} \otimes A_{2}$ under $\delta$ and the continuity of this map.

Notations. Let us now collect together some of the notations that will be used throughout the paper. The symbol $\mathscr{H}$, with or without subscripts, will denote a Hilbert space. The space of bounded linear operators on $\mathscr{H}$ will be denoted by $\mathscr{L}(\mathscr{H})$, and the space of compact linear operators on $\mathscr{H}$ will be denoted by $\mathcal{K}(\mathscr{H})$. We will denote by $\Sigma$ the set $\{1,2, \ldots, 2 \ell+1\}$ and by $\Sigma_{\ell}$ and $\Sigma_{j, \ell}$ the subsets $\{1,2, \ldots, \ell+1\}$ and $\{\ell-j+1, \ell-j+2, \ldots, \ell+1\}$, respectively, where $0 \leq j \leq \ell$.

Let $\Gamma \equiv \Gamma_{\Sigma}$ denote the set of maps $\gamma$ from $\Sigma$ to $\mathbb{Z}$ such that $\gamma_{i} \in \mathbb{N}$ for all $i \in \Sigma \backslash\{\ell+1\}$, i.e., $\Gamma_{\Sigma}=\mathbb{N}^{\ell} \times \mathbb{Z} \times \mathbb{N}^{\ell}$. For a subset $A$ of $\Sigma$, we will denote by $\gamma_{A}$ the restriction $\left.\gamma\right|_{A}$ of $\gamma$ to $A$. Let $\Gamma_{A}$ denote the set $\left\{\gamma_{A}: \gamma \in \Gamma\right\}$ and $\mathscr{H}_{A}$ be the 
Hilbert space $\ell_{2}\left(\Gamma_{A}\right)$. We will denote $\mathscr{H}_{\Sigma}$ by just $\mathscr{H}$, and $\mathscr{H}_{\Sigma_{j, \ell}}$ by $\mathscr{H}_{j}$. Thus

$$
\begin{aligned}
\mathscr{H}_{\Sigma} & =\underbrace{\ell_{2}(\mathbb{N}) \otimes \cdots \otimes \ell_{2}(\mathbb{N})}_{\ell \text { copies }} \otimes \ell_{2}(\mathbb{Z}) \otimes \underbrace{\ell_{2}(\mathbb{N}) \otimes \cdots \otimes \ell_{2}(\mathbb{N})}_{\ell \text { copies }}, \\
\mathscr{H}_{j} & =\underbrace{\ell_{2}(\mathbb{N}) \otimes \cdots \otimes \ell_{2}(\mathbb{N})}_{j \text { copies }} \otimes \ell_{2}(\mathbb{Z}) .
\end{aligned}
$$

Note that $\mathscr{H}_{j}$ and $\mathscr{H}_{\{j\}}$ are different.

Let $A \subseteq \Sigma$. We will denote by $\left\{e_{\gamma}\right\}_{\gamma}$ the natural orthonormal basis for $\mathscr{H}_{A}=$ $\ell_{2}\left(\Gamma_{A}\right)$ and by $p_{\gamma}$ the rank one projection $\left|e_{\gamma}\right\rangle\left\langle e_{\gamma}\right|$. For $i \in A$, we will denote by $N_{i}$ the number operator on the $i$-th coordinate on $\mathscr{H}_{A}$, i.e.,

$$
N_{i} \equiv \sum_{\gamma} \gamma_{i} p_{\gamma}: e_{\gamma} \mapsto \gamma_{i} e_{\gamma} \quad \text { (defined on } \mathscr{H}_{A} \text { with } i \in A \text { ). }
$$

We will denote by $\left|D_{A}\right|$ the operator $\sum_{i \in A}\left|N_{i}\right|$ on $\mathscr{H}_{A}$.

Let $F_{0}$ be the following operator on $\ell_{2}(\mathbb{Z})$ :

$$
F_{0} e_{k}= \begin{cases}e_{k} & \text { if } k \geq 0 \\ -e_{k} & \text { if } k<0\end{cases}
$$

For $1 \leq j \leq 2 \ell+1$, let $V_{j}$ be the operator on $\mathscr{H}_{\{j\}}$ defined by

$$
V_{j}:= \begin{cases}F_{0} & \text { if } j=\ell+1, \\ I & \text { otherwise. }\end{cases}
$$

Let $F_{A}$ denote the operator $\otimes_{j \in A} V_{j}$ on $\mathscr{H}_{A}$ and let $D_{A}=F_{A}\left|D_{A}\right|$. Thus

$$
D_{A} e_{\gamma}= \begin{cases}-\left(\sum_{i \in A}\left|\gamma_{i}\right|\right) e_{\gamma} & \text { if } \ell+1 \in A \text { and } \gamma_{\ell+1}<0, \\ \left(\sum_{i \in A}\left|\gamma_{i}\right|\right) e_{\gamma} & \text { otherwise. }\end{cases}
$$

We will denote $F_{\Sigma_{j, \ell}}$ by $F_{j}$ and $D_{\Sigma_{j, \ell}}$ by $D_{j}$.

Recall that $\mathscr{H}_{\{j\}}$ is $\ell_{2}(\mathbb{N})$ if $j \neq \ell+1$ and is $\ell_{2}(\mathbb{Z})$ if $j=\ell+1$. Suppose that $\mathcal{F}_{j}$ is a subspace of $\mathscr{L}\left(\mathscr{H}_{\{j\}}\right)$ for each $j \in \Sigma$. For $A \subseteq \Sigma$, define

$$
\mathscr{F}_{j, A}= \begin{cases}\mathscr{F}_{j} & \text { if } j \in A, \\ \mathbb{C} \cdot I & \text { if } j \notin A,\end{cases}
$$

and $\mathscr{F}_{A}$ to be the tensor product $\bigotimes_{j \in \Sigma} \mathscr{F}_{j, A}$ in $\mathscr{L}\left(\mathscr{H}_{\Sigma}\right)$ (the type of the tensor product will depend on the specific $\mathscr{F}_{j}$ 's we look at). This tensor product will often be identified with $\bigotimes_{j \in A} \widetilde{F}_{j} \subseteq \mathscr{L}\left(\mathscr{H}_{A}\right)$.

On both $\ell_{2}(\mathbb{N})$ and $\ell_{2}(\mathbb{Z})$, we will denote by $N$ the number operator defined by $N e_{n}=n e_{n}$ and by $S$ the left shift defined by $S e_{n}=e_{n-1}$. For $k \in \mathbb{Z}$ (for 
$k \in \mathbb{N}$ in the case of $\left.\ell_{2}(\mathbb{N})\right)$, let $p_{k}$ denote the projection $\left|e_{k}\right\rangle\left\langle e_{k}\right|$. We will freely identify $\ell_{2}(\mathbb{Z})$ with $L_{2}(\mathbb{T})$. Thus the right shift on $\ell_{2}(\mathbb{Z})$ will be multiplication by the function $t \mapsto t$ and will be denoted by $z$. Let $\mathcal{T}$ be the Toeplitz algebra, i.e., the $\mathrm{C}^{*}$-subalgebra of $\mathscr{L}\left(\ell_{2}(\mathbb{N})\right)$ generated by $S$. For a positive integer $k$, we will denote by $\mathcal{T}_{k}$ the $k$-fold tensor product of $\mathcal{T}$, embedded in $\mathscr{L}\left(\ell_{2}\left(\mathbb{N}^{k}\right)\right)$. Denote by $\sigma$ the symbol map from $\mathcal{T}$ to $C(\mathbb{T})$ that sends $S^{*}$ to $z$ and all compact operators to 0 .

\section{Torus equivariant spectral triple}

3.1. The spectral triple. In this section we recall the spectral triple for the odddimensional quantum spheres given in [3]. We begin with some known facts about odd-dimensional quantum spheres. Let $q \in[0,1]$. The $\mathrm{C}^{*}$-algebra $C\left(S_{q}^{2 \ell+1}\right)$ of the quantum sphere $S_{q}^{2 \ell+1}$ is the universal $\mathrm{C}^{*}$-algebra generated by elements $z_{1}, z_{2}, \ldots, z_{\ell+1}$ satisfying the following relations (see [10]):

$$
\begin{aligned}
z_{i} z_{j} & =q z_{j} z_{i}, & & 1 \leq j<i \leq \ell+1, \\
z_{i}^{*} z_{j} & =q z_{j} z_{i}^{*}, & & 1 \leq i \neq j \leq \ell+1, \\
z_{i} z_{i}^{*}-z_{i}^{*} z_{i}+\left(1-q^{2}\right) \sum_{k>i} z_{k} z_{k}^{*} & =0, & & 1 \leq i \leq \ell+1, \\
\sum_{i=1}^{\ell+1} z_{i} z_{i}^{*} & =1 . & &
\end{aligned}
$$

We will denote by $\mathcal{A}\left(S_{q}^{2 \ell+1}\right)$ the *-subalgebra of $A_{\ell}$ generated by the $z_{j}$ 's. Note that for $\ell=0$, the $\mathrm{C}^{*}$-algebra $C\left(S_{q}^{2 \ell+1}\right)$ is the algebra of continuous functions $C(\mathbb{T})$ on the torus and for $\ell=1$, it is $C\left(\mathrm{SU}_{q}(2)\right)$.

There is a natural torus group $\mathbb{T}^{\ell+1}$ action $\tau$ on $C\left(S_{q}^{2 \ell+1}\right)$ as follows. For $w=$ $\left(w_{1}, \ldots, w_{\ell+1}\right)$, define an automorphism $\tau_{w}$ by $\tau_{w}\left(z_{i}\right)=w_{i} z_{i}$. Let $Y_{k, q}$ be the following operators on $\mathscr{H}_{\ell}$ :

$$
Y_{k, q}= \begin{cases}\underbrace{q^{N} \otimes \cdots \otimes q^{N}}_{k-1 \text { copies }} \otimes \sqrt{1-q^{2 N}} S^{*} \otimes \underbrace{I \otimes \cdots \otimes I}_{\ell+1-k \text { copies }} & \text { if } 1 \leq k \leq \ell, \\ \underbrace{q^{N} \otimes \cdots \otimes q^{N}}_{\ell \text { copies }} \otimes S^{*} & \text { if } k=\ell+1 .\end{cases}
$$

Here for $q=0, q^{N}$ stands for the rank one projection $p_{0}=\left|e_{0}\right\rangle\left\langle e_{0}\right|$. Then $\pi_{\ell}: z_{k} \mapsto$ $Y_{k, q}$ gives a faithful representation of $C\left(S_{q}^{2 \ell+1}\right)$ on $\mathscr{H}_{\ell}$ for $q \in[0,1)$ (see Lemma 4.1 and Remark 4.5, [10]). We denote the image $\pi_{\ell}\left(C\left(S_{q}^{2 \ell+1}\right)\right)$ by $A_{\ell}(q)$ or by just $A_{\ell}$.

Let $\left\{e_{\gamma}: \gamma \in \Gamma_{\Sigma_{\ell}}\right\}$ be the standard orthonormal basis for $\mathscr{H}_{\ell}$. For $w=$ $\left(w_{1}, w_{2}, \ldots, w_{\ell+1}\right)$, define $U_{w}\left(e_{\gamma}\right)=w_{1}^{\gamma_{1}} w_{2}^{\gamma_{2}} \ldots w_{\ell+1}^{\gamma_{\ell+1}} e_{\gamma}$ to be the unitary $U_{w}$ on $\mathscr{H}_{\ell}$, where $\gamma=\left(\gamma_{1}, \gamma_{2}, \ldots, \gamma_{\ell+1}\right) \in \Gamma_{\Sigma_{\ell}}$. Then $\left(\pi_{\ell}, U\right)$ is a covariant representation of $\left(C\left(S_{q}^{2 \ell+1}\right), \mathbb{T}^{\ell+1}, \tau\right)$. Note that $A_{\ell} \subset \mathcal{T}_{\ell} \otimes C(\mathbb{T})$. 
In [3] all spectral triples equivariant with respect to this covariant representation were characterised, and an optimal one was constructed. We recall the following theorem from [3].

Theorem 3.1 ([3]). Let $D_{\ell}$ be the operator $e_{\gamma} \rightarrow d(\gamma) e_{\gamma}$ on $\mathscr{H}_{\ell}$, where the $d_{\gamma}$ 's are given by

$$
d(\gamma)= \begin{cases}\gamma_{1}+\gamma_{2}+\ldots \gamma_{\ell}+\left|\gamma_{\ell+1}\right| & \text { if } \gamma_{\ell+1} \geq 0, \\ -\left(\gamma_{1}+\gamma_{2}+\ldots \gamma_{\ell}+\left|\gamma_{\ell+1}\right|\right) & \text { if } \gamma_{\ell+1}<0 .\end{cases}
$$

Then $\left(\mathcal{A}\left(S_{q}^{2 \ell+1}\right), \mathscr{H}_{\ell}, D_{\ell}\right)$ is a non-trivial $(\ell+1)$-summable spectral triple. Also $D_{\ell}$ commutes with $U_{w}$ for every $w \in \mathbb{T}^{\ell+1}$.

The operator $D_{\ell}$ is optimal, i.e., if $\left(\mathcal{A}\left(S_{q}^{2 \ell+1}\right), \mathcal{H}_{\ell}, D\right)$ is a spectral triple such that $D$ commutes with $U_{w}$ for every $w$, then there exist positive real numbers a and $b$ such that $|D| \leq a+b\left|D_{\ell}\right|$.

In the next few sections, we will introduce a dense subalgebra $\mathcal{A}_{\ell}^{\infty}$ of $A_{\ell}(q)$ closed under its holomorphic function calculus and establish regularity of the spectral triple $\left(\mathcal{A}_{\ell}^{\infty}, \mathcal{H}_{\ell}, D_{\ell}\right)$. We will also compute its dimension spectrum.

3.2. The smooth function algebra $\mathcal{A}_{\ell}^{\infty}$. In this section we associate a dense Fréchet $\mathrm{C}^{*}$-subalgebra of $A_{\ell}(q)=\pi_{\ell}\left(C\left(S_{q}^{2 \ell+1}\right)\right)$ which is closed under holomorphic functional calculus. We first show that the $\mathrm{C}^{*}$-algebra $A_{\ell}(q)$ is independent of $q$.

Lemma 3.2. For any $q \in(0,1)$, one has $A_{\ell}(0)=A_{\ell}(q)$.

Proof. Let us first show that $A_{\ell}(q) \subseteq A_{\ell}(0)$. We prove this by induction on $\ell$. Let us denote the generators $Y_{j, q}$ of $A_{\ell}(q)$ by $Y_{j, q}^{(\ell+1)}$. Note that for $\ell=0$, one has $Y_{1, q}^{(1)}=$ $Y_{1,0}^{(1)}$ and $A_{0}(q)=A_{0}(0)=C(\mathbb{T})$ so that the inclusion is trivial. Next, assume the inclusion for $\ell-1$. Observe that for $1 \leq j \leq \ell$, we have $Y_{j+1, q}^{(\ell+1)}=q^{N} \otimes Y_{j, q}^{(\ell)}$ and $Y_{j+1,0}^{(\ell+1)}=p_{0} \otimes Y_{j, 0}^{(\ell)}$. From this last equality and from the induction hypothesis, it follows that $p_{0} \otimes Y_{j, q}^{(\ell)} \in A_{\ell}(0)$ for $1 \leq j \leq \ell$. Since for $1 \leq j \leq \ell$,

$$
Y_{j+1, q}^{(\ell+1)}=q^{N} \otimes Y_{j, q}^{(\ell)}=\sum_{n \in \mathbb{N}} q^{n}\left(Y_{1,0}^{(\ell+1)}\right)^{n}\left(p_{0} \otimes Y_{j, q}^{(\ell)}\right)\left(Y_{1,0}^{(\ell+1)}\right)^{* n},
$$

it follows that $Y_{j, q}^{(\ell+1)} \in A_{\ell}(0)$ for $2 \leq j \leq \ell+1$. So it remains to show that $Y_{1, q}^{(\ell+1)} \in A_{\ell}(0)$. Note that $Y_{1, q}^{(\ell+1)}=\left(\sqrt{I-q^{2 N}} \otimes I\right) Y_{1,0}^{(\ell+1)}$ and

$$
\begin{aligned}
q^{N} \otimes I & =\sum \sum_{n \in \mathbb{N}} q^{n}\left(Y_{1,0}^{(\ell+1)}\right)^{n}\left(p_{0} \otimes I\right)\left(Y_{1,0}^{(\ell+1)}\right)^{* n} \\
& =\sum \sum_{n \in \mathbb{N}} q^{n}\left(Y_{1,0}^{(\ell+1)}\right)^{n}\left(Y_{2,0}^{(\ell+1)}\right)^{*}\left(Y_{2,0}^{(\ell+1)}\right)\left(p_{0} \otimes I\right)\left(Y_{1,0}^{(\ell+1)}\right)^{* n} .
\end{aligned}
$$


Therefore we have the required inclusion.

For the other inclusion, we will use the following fact: if $B$ denotes the $\mathrm{C}^{*}$ subalgebra of $\mathscr{L}\left(\ell_{2}(\mathbb{N})\right)$ generated by the operator $X=\left(1-q^{2 N}\right)^{\frac{1}{2}} S^{*}$, then $B$ contains the shift operator $S$. This is because the operator $|X|$ is invertible and $S^{*}=X|X|^{-1}$. Using this fact for the first copy of $\ell_{2}(\mathbb{N})$, since $Y_{1, q} \in A_{\ell}(q)$, one gets $Y_{1,0} \in A_{\ell}(q)$. Next assume that $Y_{i, 0} \in A_{\ell}(q)$ for $1 \leq i \leq j-1$, where $2 \leq j \leq \ell$. Then $P_{j-1}:=I-\sum_{k=1}^{j-1} Y_{k, 0} Y_{k, 0}^{*} \in A_{\ell}(q)$. Observe that

$$
\begin{aligned}
P_{j-1} Y_{j, q} & =\underbrace{p_{0} \otimes \cdots \otimes p_{0}}_{j-1} \otimes X \otimes \underbrace{I \otimes \cdots \otimes I}_{\ell+1-j}, \\
Y_{j, 0} & =\underbrace{p_{0} \otimes \cdots \otimes p_{0}}_{j-1} \otimes S^{*} \otimes \underbrace{I \otimes \cdots \otimes I}_{\ell+1-j} .
\end{aligned}
$$

Therefore using the above fact for the $j$-th copy of $\ell_{2}(\mathbb{N})$ we get $Y_{j, 0} \in A_{\ell}(q)$. Finally, since $Y_{\ell+1,0}=Y_{\ell+1, q}\left(I-\sum_{k=1}^{\ell} Y_{k, 0} Y_{k, 0}^{*}\right)$, one has $Y_{\ell+1,0} \in A_{\ell}(q)$.

Write $\alpha_{i}$ for $Y_{i, 0}^{*}$. Note that the $\mathrm{C}^{*}$-subalgebra of $A_{\ell}$ generated by $\alpha_{2}, \ldots, \alpha_{\ell+1}$ is isomorphic to $A_{\ell-1}$ where the map $a \mapsto p_{0} \otimes a$ gives the isomorphism. We define the Fréchet subalgebras $\mathscr{A}_{\ell}^{\infty}$ inductively as follows.

The algebra

$$
\mathcal{A}_{0}^{\infty}:=\left\{\sum_{n \in \mathbb{Z}} a_{n} z^{n}:\left(a_{n}\right) \text { is rapidly decreasing }\right\}
$$

is the algebra of smooth functions on $\mathbb{T}$ together with the increasing family of seminorms $\|\cdot\|_{p}$ given by $\left\|\left(a_{n}\right)\right\|_{p}=\sum(1+|n|)^{p}\left|a_{n}\right|$. Then $\mathcal{A}_{0}^{\infty}$ is a dense Fréchet $\mathrm{C}^{*}$-subalgebra of $A_{0}=C(\mathbb{T})$. Note that $\|a\| \leq\|a\|_{0}$ for $a \in \mathcal{A}_{0}^{\infty}$. Now assume $\left(\mathcal{A}_{\ell-1}^{\infty},\|\cdot\|_{m}\right)$ be defined such that

(1) the seminorms $\|\cdot\|_{m}$ are increasing and $\left(\mathcal{A}_{\ell-1}^{\infty},\|\cdot\|_{m}\right)$ is a Fréchet algebra,

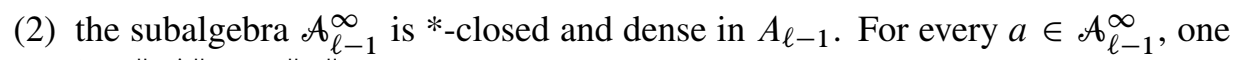
has $\left\|a^{*}\right\|_{m}=\|a\|_{m}$,

(3) for every $a \in \mathcal{A}_{\ell-1}^{\infty}$, one has $\|a\| \leq\|a\|_{0}$, where $\|\cdot\|$ denotes the $\mathrm{C}^{*}$ norm of $A_{\ell-1}$.

Now define

$$
\begin{aligned}
\mathcal{A}_{\ell}^{\infty}:=\{ & \left\{\sum_{j, k \in \mathbb{N}} \alpha_{1}^{* j}\left(p_{0} \otimes a_{j k}\right) \alpha_{1}^{k}+\sum_{k \geq 0} \lambda_{k} \alpha_{1}^{k}+\sum_{k>0} \lambda_{-k} \alpha_{1}^{* k}:\right. \\
& a_{j k} \in \mathcal{A}_{\ell-1}^{\infty}, \sum_{j, k}(1+j+k)^{n}\left\|a_{j k}\right\|_{m}<\infty,\left(\lambda_{k}\right) \text { is } \\
& \text { rapidly decreasing }\} .
\end{aligned}
$$

Let $a:=\sum_{j, k} \alpha_{1}^{* j}\left(p_{0} \otimes a_{j k}\right) \alpha_{1}^{k}+\sum_{k \geq 0} \lambda_{k} \alpha_{1}^{k}+\sum_{k>0} \lambda_{-k} \alpha_{1}^{* k}$ be an element of $\mathcal{A}_{\ell}^{\infty}$. Define for $m \in \mathbb{N}$, the seminorms $\|a\|_{m}$ as

$$
\|a\|_{m}=\max _{r, s \leq m}\left(\sum_{j, k}(1+j+k)^{r}\left\|a_{j k}\right\|_{s}\right)+\sum_{k \in \mathbb{Z}}(1+|k|)^{m}\left|\lambda_{k}\right| .
$$


Proposition 3.3. The pair $\left(\mathcal{A}_{\ell}^{\infty},\|\cdot\|_{m}\right)$ has the following properties:

(1) the seminorms $\|\cdot\|_{m}$ are increasing and $\left(\mathcal{A}_{\ell}^{\infty},\|\cdot\|_{m}\right)$ is a Fréchet algebra,

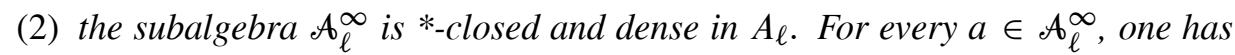
$\left\|a^{*}\right\|_{m}=\|a\|_{m}$

(3) for every $a \in \mathcal{A}_{\ell}^{\infty}$, one has $\|a\| \leq\|a\|_{0}$ where $\|\cdot\|$ denotes the $C^{*}$ norm of $A_{\ell}$.

Proof. The proof is by induction on $\ell$. Parts (2) and (3) and the fact that the seminorms $\|\cdot\|_{m}$ are increasing follow from the definition and the induction hypothesis. One verifies directly that $\left(\mathcal{A}_{\ell}^{\infty},\|\cdot\|_{m}\right)$ is a Fréchet algebra using induction and the relations

$$
\begin{gathered}
\alpha_{1} \alpha_{1}^{*}=1, \\
\alpha_{1}^{* j}\left(p_{0} \otimes a_{j k}\right) \alpha_{1}^{k} \alpha_{1}^{* r}\left(p_{0} \otimes a_{r s}\right) \alpha_{1}^{s}=\delta_{k r} \alpha_{1}^{* j}\left(p_{0} \otimes a_{j k} a_{r s}\right) \alpha_{1}^{s}, \\
\alpha_{1}^{* m} \alpha_{1}^{n}= \begin{cases}\left(\alpha_{1}^{*}\right)^{m-n}-\sum_{k=0}^{n-1}\left(\alpha_{1}^{*}\right)^{m-n+k}\left(p_{0} \otimes 1\right) \alpha_{1}^{k} & \text { if } m \geq n, \\
\alpha_{1}^{n-m}-\sum_{k=0}^{m-1} \alpha_{1}^{* k}\left(p_{0} \otimes 1\right) \alpha_{1}^{n-m+k} & \text { if } m<n .\end{cases}
\end{gathered}
$$

Denote the generators $z_{1}, z_{2}, \ldots, z_{\ell+1}$ of $C\left(S_{q}^{2 \ell+1}\right)$ by $z_{1}^{(\ell+1)}, z_{2}^{(\ell+1)}, \ldots, z_{\ell+1}^{(\ell+1)}$. Let $\sigma_{\ell}: C\left(S_{q}^{2 \ell+1}\right) \rightarrow C\left(S_{q}^{2 \ell-1}\right)$ be the homomorphism given by $\sigma_{\ell}\left(z_{\ell+1}^{(\ell+1)}\right)=0$ and $\sigma_{\ell}\left(z_{i}^{(\ell+1)}\right)=z_{i}^{(\ell)}$ for $1 \leq i \leq \ell$. Let us denote by the same symbol $\sigma_{\ell}$ the induced homomorphism from $A_{\ell}$ to $A_{\ell-1}$. Observe that if one applies the map $\sigma$ on the $\ell$-th copy of $\mathcal{T}$ in $\mathcal{T}_{\ell} \otimes C(\mathbb{T})$ followed by evaluation at 1 in the $(\ell+1)$-th copy, then the restriction of the resulting map to $A_{\ell}$ is precisely $\sigma_{\ell}$.

Proposition 3.4. The dense Fréchet $C^{*}$-subalgebra $\mathcal{A}_{\ell}^{\infty}$ of $A_{\ell}$ is closed under holomorphic functional calculus in $A_{\ell}$. Moreover, the algebra $\mathcal{A}_{\ell}^{\infty}$ contains the generators $Y_{1, q}^{(\ell+1)}, \ldots, Y_{\ell+1, q}^{(\ell+1)}$.

Proof. We prove this proposition by induction on $\ell$. For $\ell=0$, by definition $\mathcal{A}_{0}^{\infty}=C^{\infty}(\mathbb{\mathbb { T }})$. Hence the proposition is clear in this case. Now assume that the algebra $\mathcal{A}_{\ell-1}^{\infty}$ is closed under holomorphic functional calculus in $A_{\ell-1}$ and contains $Y_{1, q}^{(\ell)}, \ldots, Y_{\ell, q}^{(\ell)}$. The homomorphism $\sigma_{\ell}: A_{\ell} \rightarrow A_{\ell-1}$ gives the exact sequence

$$
0 \longrightarrow \mathcal{K}\left(\ell_{2}\left(\mathbb{N}^{\ell}\right)\right) \otimes C(\mathbb{T}) \longrightarrow A_{\ell} \longrightarrow A_{\ell-1} \longrightarrow 0
$$

One also has at the smooth algebra level the "sub" extension

$$
0 \longrightarrow S\left(\ell_{2}\left(\mathbb{N}^{\ell}\right)\right) \otimes C^{\infty}(\mathbb{T}) \longrightarrow \mathcal{A}_{\ell}^{\infty} \longrightarrow \mathcal{A}_{\ell-1}^{\infty} \longrightarrow 0
$$

Since $S\left(\ell_{2}\left(\mathbb{N}^{\ell}\right)\right) \otimes C^{\infty}(\mathbb{\mathbb { T }}) \subset \mathcal{K}\left(\ell_{2}\left(\mathbb{N}^{\ell}\right)\right) \otimes C(\mathbb{T})$ and $\mathcal{A}_{\ell-1}^{\infty} \subset A_{\ell-1}$ are closed under the respective holomorphic functional calculus, it follows from Theorem 3.2, part 2, [13] that $\mathcal{A}_{\ell}^{\infty}$ is spectrally invariant in $A_{\ell}$. Since $\|a\| \leq\|a\|_{0}$ for all $a \in$ $\mathcal{A}_{\ell}^{\infty}$, it follows that the Fréchet topology of $\mathcal{A}_{\ell}^{\infty}$ is finer than the norm topology. 
Therefore $\mathcal{A}_{\ell}^{\infty}$ is closed under holomorphic functional calculus in $A_{\ell}$. Observe that $Y_{i, q}^{(\ell+1)}=\sum_{n \geq 0} q^{n} \alpha_{1}^{* n}\left(p_{0} \otimes Y_{i-1, q}^{(\ell)}\right) \alpha_{1}^{n}$ for $i \geq 2$. Hence $Y_{i, q}^{(\ell+1)} \in \mathcal{A}_{\ell}^{\infty}$ for $i=$ $2, \ldots, \ell+1$. Also note that $q^{N} \otimes I=\sum_{n \geq 0} q^{n} \alpha_{1}^{* n}\left(p_{0} \otimes 1\right) \alpha_{1}$. Since $\mathcal{A}_{\ell}^{\infty}$ is closed under holomorphic functional calculus, it follows that $\sqrt{1-q^{2 N+2}} \otimes I \in \mathcal{A}_{\ell}^{\infty}$. As $Y_{1, q}^{(\ell+1)}=\alpha_{1}^{*}\left(\sqrt{1-q^{2 N+2}} \otimes I\right)$ it follows that $Y_{1, q}^{(\ell+1)} \in \mathcal{A}_{\ell}^{\infty}$. This completes the proof.

Next we proceed to prove that the spectral triple $\left(\mathcal{A}_{\ell}^{\infty}, \mathscr{H}_{\ell}, D_{\ell}\right)$ is regular and compute its dimension spectrum. The proof is by induction. We start with the case $\ell=0$ to start the induction.

3.3. The case $\ell=0$. For $\ell=0$, the spectral triple $\left(\mathcal{A}_{0}^{\infty}, \mathscr{H}_{0}, D_{0}\right)$ is unitarily equivalent to the spectral triple $\left(C^{\infty}(\mathbb{T}), L_{2}(\mathbb{T}), \frac{1}{i} \frac{d}{d \theta}\right)$. For $f \in C^{\infty}(\mathbb{T})$ one has $\left[D_{0}, f\right]=\frac{1}{i} f^{\prime}$. Let $\left(e_{k}\right)$ be the standard orthonormal basis and let $p_{k}$ be the projection onto $e_{k}$. Let $F_{0}:=\operatorname{sign}\left(D_{0}\right)$. Note that $\left[F_{0}, z\right]=2 p_{0} z$ and hence by induction $\left[F_{0}, z^{n}\right]=2 \sum_{k=0}^{n-1} p_{k} z^{n} p_{k-n}$ for $n \geq 0$. Thus $\left[F_{0}, z^{n}\right]$ is smoothing for $n \geq 0$. Also $\left\|\left|D_{0}\right|^{r}\left[F_{0}, z^{n}\right]\left|D_{0}\right|^{s}\right\| \leq 2(1+n)^{r+s+1}$. Since $\left[F_{0}, z^{-|n|}\right]^{*}=$ $-\left[F_{0}, z^{|n|}\right]$, it follows that $\left[F_{0}, z^{n}\right] \in \mathrm{OP}^{-\infty}$ for every $n$. Moreover, $\left\|\left[F_{0}, z^{n}\right]\right\|_{r, s} \leq$ $2(1+|n|)^{r+s+1}$. Hence we observe that $\left[F_{0}, f\right] \in \mathrm{OP}^{-\infty}$ and $\left\|\left[F_{0}, f\right]\right\|_{r, s} \leq$ $2\|f\|_{r+s+1}$. Let $\delta$ be the unbounded derivation $\left[\left|D_{0}\right|, \cdot\right]$.

Lemma 3.5. Let $\mathscr{B}:=\left\{f_{0}+f_{1} F_{0}+R: f_{0}, f_{1} \in C^{\infty}(\mathbb{T}), R \in \mathrm{OP}_{D_{0}}^{-\infty}\right\}$. Then:

(1) If $f_{0}+f_{1} F_{0}$ is smoothing then $f_{0}=f_{1}=0$. Hence $B$ is isomorphic to the direct sum $C^{\infty}(\mathbb{T}) \oplus C^{\infty}(\mathbb{T}) \oplus \mathrm{OP}_{D_{0}}^{-\infty}$. We give $\mathscr{B}$ the Fréchet space structure coming from this decomposition. This topology on $\mathscr{B}$ is generated by the seminorms $\left(\|\cdot\|_{m}\right)_{m \in \mathbb{N}}$ which are defined by $\left\|f_{0}+f_{1} F_{0}+R\right\|_{m}:=$ $\left\|f_{0}\right\|_{m}+\left\|f_{1}\right\|_{m}+\sum_{r+s \leq m}\|R\|_{r, s}$.

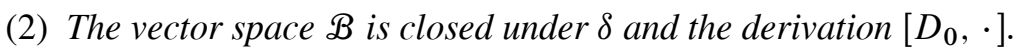

(3) For every $b \in \mathscr{B},\left[F_{0}, b\right] \in \mathrm{OP}^{-\infty}$. Also the map $b \rightarrow\left[F_{0}, b\right] \in \mathrm{OP}^{-\infty}$ is continuous. The derivations $\delta$ and $\left[D_{0}, \cdot\right]$ are continuous.

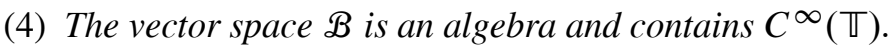

Proof. First observe that a bounded operator $T$ on $\ell_{2}(\mathbb{Z})$ is smoothing if and only if $\left(\left\langle T e_{m}, e_{n}\right\rangle\right)_{m, n}$ is rapidly decreasing. Now suppose $R:=f_{0}+f_{1} F_{0}$ be smoothing. Fix an integer $r$. Observe that $\left\langle R\left(e_{n}\right), e_{r+n}\right\rangle$ converges to $\hat{f}_{0}(r)+\hat{f}_{1}(r)$ as $n \rightarrow+\infty$ and converges to $\hat{f}_{0}(r)-\hat{f}_{1}(r)$ as $n \rightarrow-\infty$. But since $R$ is smoothing it follows that $\hat{f}_{0}(r)+\hat{f}_{1}(r)=0=\hat{f}_{0}(r)-\hat{f}_{1}(r)$. Hence $\hat{f}_{0}(r)=\hat{f}_{1}(r)=0$ for every integer $r$. Thus $f_{0}=f_{1}=0$. This proves part (1).

Parts (2), (3) and (4) follow from the observations that $\left[D_{0}, f\right]=\frac{1}{i} f^{\prime},\left[F_{0}, f\right] \in$ $\mathrm{OP}^{-\infty},\left\|\left[F_{0}, f\right]\right\|_{r, s} \leq 2\|f\|_{r+s+1}$ and $\delta(b)=\left[D_{0}, b\right] F_{0}+D_{0}\left[F_{0}, b\right]$. This completes the proof. 
In particular, it follows from parts (2) and (4) of the above lemma that the spectral triple $\left(\mathcal{A}_{0}^{\infty}, \mathscr{H}_{0}, D_{0}\right)$ is regular.

Let $\mathcal{E}$ be the $\mathrm{C}^{*}$-subalgebra of $\mathscr{L}\left(\ell_{2}(\mathbb{Z})\right)$ generated by $C(\mathbb{T})$ and $F_{0}$. Note that the algebra $\mathscr{B}$ plays the role of smooth function subalgebra for the $\mathrm{C}^{*}$-algebra $\mathcal{E}$. Therefore $\mathcal{E}^{\infty}$ will stand for the algebra $\mathcal{B}$.

3.4. Regularity and the dimension spectrum. In this section we prove regularity and calculate the dimension spectrum for the spectral triple $\left(\mathcal{A}_{\ell}^{\infty}, \mathscr{H}_{\ell}, D_{\ell}\right)$. The proof is by induction on $\ell$. Let us denote the derivation $\left[\left|D_{\ell}\right|, \cdot\right]$ by $\delta_{\ell}$ and let $F_{\ell}$ stand for the sign of the operator $D_{\ell}$. Observe that $F_{\ell}=1^{\otimes \ell} \otimes F_{0}=1 \otimes F_{\ell-1}$.

Proposition 3.6. Let $\mathscr{B}_{\ell}:=\left\{A_{0}+A_{1} F_{\ell}+R: A_{0}, A_{1} \in \mathcal{A}_{\ell}^{\infty}, R \in \mathrm{OP}^{-\infty}\right\}$. Then:

(1) If $A_{0}+A_{1} F_{\ell}$ is smoothing then $A_{0}=A_{1}=0$. Hence $\mathcal{B}_{\ell}$ is isomorphic to the direct sum $\mathcal{A}_{\ell}^{\infty} \oplus \mathcal{A}_{\ell}^{\infty} \oplus \mathrm{OP}^{-\infty}$. Equip $\mathscr{B}_{\ell}$ with the Fréchet space structure coming from this decomposition. This topology on $\mathfrak{B}_{\ell}$ is induced by the seminorms $\left(\|\cdot\|_{m}\right)_{m \in \mathbb{N}}$ which are defined by $\left\|A_{0}+A_{1} F_{\ell}+R\right\|_{m}:=$ $\left\|A_{0}\right\|_{m}+\left\|A_{1}\right\|_{m}+\sum_{r+s \leq m}\|R\|_{r, s}$.

(2) For every $b \in \mathcal{B}_{\ell},\left[F_{\ell}, b\right] \in \mathrm{OP}^{-\infty}$. Also the map $b \rightarrow\left[F_{\ell}, b\right] \in \mathrm{OP}^{-\infty}$ is continuous.

(3) The vector space $\mathfrak{B}_{\ell}$ is closed under the derivations $\delta_{\ell}$ and $\left[D_{\ell}, \cdot\right]$. Moreover the derivations $\delta_{\ell}$ and $\left[D_{\ell}, \cdot\right]$ are continuous.

(4) The vector space $\mathfrak{B}_{\ell}$ is an algebra and contains $\mathcal{A}_{\ell}^{\infty}$.

Proof. The proof is by induction on $\ell$. For $\ell=0$, the proposition is just Lemma 3.5. Now assume that the proposition is true for $\ell-1$. Suppose that $A_{0}+A_{1} F_{\ell}$ is smoothing for some $A_{0}, A_{1} \in \mathcal{A}_{\ell}^{\infty}$. Then $A_{0}+A_{1} F_{\ell} \in \mathcal{T}_{\ell} \otimes \mathscr{E}$ and $A_{0}+A_{1} F_{\ell}$ is compact. Therefore $(\sigma \otimes \mathrm{id})\left(A_{0}+A_{1} F_{\ell}\right)=0$. Now let

$$
A_{i}=\sum_{j, k \geq 0} \alpha_{1}^{* j}\left(p_{0} \otimes a_{j k}^{(i)}\right) \alpha_{1}^{k}+\sum_{k \geq 0} \lambda_{k}^{(i)} \alpha_{1}^{k}+\sum_{k>0} \lambda_{-k}^{(i)} \alpha_{1}^{* k}
$$

for $i=0,1$. Let $f_{i}(z)=\sum_{k \in \mathbb{Z}} \lambda_{k}^{(i)} z^{k}$ for $i=0,1$. Now $(\sigma \otimes i d)\left(A_{0}+A_{1} F_{\ell}\right)=$ $f_{0} \otimes I+f_{1} \otimes F_{\ell-1}$. So we have $f_{0} \otimes I+f_{1} \otimes F_{\ell-1}=0$. Writing $F_{\ell}=2 P_{\ell}-I$, it follows that $\left(f_{0}+f_{1}\right) \otimes P_{\ell-1}+\left(f_{0}-f_{1}\right) \otimes\left(1-P_{\ell-1}\right)=0$. Hence $f_{0}=f_{1}=0$. This shows that $\lambda_{k}^{(i)}=0$ for $i=0,1$. Let $b_{j k}=a_{j k}^{0}+a_{j k}^{1} F_{\ell-1}$. Since $R:=A_{0}+A_{1} F_{\ell}$ is smoothing, it follows that for every $j, k$ the matrix entries $\left\langle e_{(j, \gamma)}, R\left(e_{\left(k, \gamma^{\prime}\right)}\right)\right\rangle$ are rapidly decreasing in $\left(\gamma, \gamma^{\prime}\right)$. Hence $b_{j k}$ is smoothing for every $j, k$. By induction hypothesis $a_{j k}^{(i)}=0$ for every $j, k \geq 0$ and for $i=0,1$. Thus $A_{0}=A_{1}=0$. This proves part (1).

Observe that

$$
\delta_{\ell}\left(\alpha_{1}\right)=-\alpha_{1}, \quad\left|D_{\ell}\right|^{r} \alpha_{1}^{* k}=\alpha_{1}^{* k}\left(\left|D_{\ell}\right|+k\right)^{r}, \quad \alpha_{1}^{k}\left|D_{\ell}\right|^{s}=\left(\left|D_{\ell}\right|+k\right)^{s} \alpha_{1}^{k} .
$$


Also $F_{\ell}$ commutes with $\alpha_{1}$. To prove (2), it is enough to show that $\left[F_{\ell}, a\right]$ is smoothing for every $a \in \mathcal{A}_{\ell}^{\infty}$ and the map $a \mapsto\left[F_{\ell}, a\right]$ is continuous. Let

$$
a=\sum_{m, n \geq 0} \alpha_{1}^{* m}\left(p_{0} \otimes a_{m n}\right) \alpha_{1}^{n}+\sum_{m \geq 0} \lambda_{m} \alpha_{1}^{m}+\sum_{m>0} \lambda_{-m} \alpha_{1}^{* m}
$$

be an element in $\mathcal{A}_{\ell}^{\infty}$. Then $\left[F_{\ell}, a\right]=\sum_{m, n \geq 0} \alpha_{1}^{* m}\left(p_{0} \otimes\left[F_{\ell-1}, a_{m n}\right]\right) \alpha_{1}^{n}$. By induction hypothesis, it follows that $p_{0} \otimes\left[F_{\ell-1}, a_{m n}\right]$ is smoothing for every $m, n \geq 0$. Since $\left(\mathrm{OP}_{D_{\ell}}^{-\infty},\|\cdot\|_{r, s}\right)$ is a Fréchet space, to show that $\left[F_{\ell}, a\right]$ is smoothing it is enough to show that the infinite sum $\sum_{m, n>0} \alpha_{1}^{* m}\left(p_{0} \otimes\left[F_{\ell-1}, a_{m n}\right]\right) \alpha_{1}^{n}$ converges absolutely in every seminorm $\|\cdot\|_{r, s}$. Now observe that

$$
\begin{aligned}
& \left|D_{\ell}\right|^{r} \alpha_{1}^{* m}\left(p_{0} \otimes\left[F_{\ell-1}, a_{m n}\right]\right) \alpha_{1}^{n}\left|D_{\ell}\right|^{s} \\
& \quad=\alpha_{1}^{* m}\left(\left|D_{\ell}\right|+m\right)^{r}\left(p_{0} \otimes\left[F_{\ell-1}, a_{m n}\right]\right)\left(\left|D_{\ell}\right|+n\right)^{s} \alpha_{1}^{n} .
\end{aligned}
$$

Since the map $a^{\prime} \in \mathcal{A}_{\ell-1}^{\infty} \mapsto\left[F_{\ell-1}, a^{\prime}\right] \in \mathrm{OP}^{-\infty}$ is continuous, there exist $p \in \mathbb{N}$ and $C_{p}>0$ such that $\left\|\left[F_{\ell-1}, a^{\prime}\right]\right\|_{i, j} \leq C_{p}\left\|a^{\prime}\right\|_{p}$ for every $a^{\prime} \in \mathcal{A}_{\ell-1}^{\infty}$ and for $i, j \leq \max \{r, s\}$. Hence, by eq. (3.3), it follows that

$$
\begin{aligned}
\sum_{m, n}\left\|\alpha_{1}^{* m}\left(p_{0} \otimes\left[F_{\ell-1}, a_{m n}\right]\right) \alpha_{1}^{n}\right\|_{r, s} & \leq \sum_{m, n} \sum_{i=0}^{r} \sum_{j=0}^{s}\left(\begin{array}{c}
r \\
i
\end{array}\right)\left(\begin{array}{c}
s \\
j
\end{array}\right) m^{r-i} n^{s-j}\left\|\left[F_{\ell-1}, a_{m n}\right]\right\|_{i, j} \\
& \leq \sum_{i=0}^{r} \sum_{j=0}^{s}\left(\begin{array}{c}
r \\
i
\end{array}\right)\left(\begin{array}{c}
s \\
j
\end{array}\right) C_{p}\left(\sum_{m, n} m^{r} n^{s}\left\|a_{m n}\right\|_{p}\right) .
\end{aligned}
$$

This shows that $\left[F_{\ell}, a\right]$ is smoothing and the above inequality also shows that for every $r, s \geq 0$, there exists $t \geq 0$ and a $C_{t}>0$ such that $\left\|\left[F_{\ell}, a\right]\right\|_{r, s} \leq C_{t}\|a\|_{t}$. Hence the map $a \mapsto\left[F_{\ell}, a\right]$ is continuous. This proves (2).

To show (3), it is enough to show that the map $a \mapsto \delta_{\ell}(a)$ from $\mathcal{A}_{\ell}^{\infty}$ to $\mathscr{B}_{\ell}$ makes sense and is continuous. We will use the fact that the unbounded derivation $\delta_{\ell}$ is a closed derivation. Let $a=\sum_{m, n \geq 0} \alpha_{1}^{* m}\left(p_{0} \otimes a_{m n}\right) \alpha_{1}^{n}+\sum_{m \geq 0} \lambda_{m} \alpha_{1}^{m}+$ $\sum_{m>0} \lambda_{-m} \alpha_{1}^{* m}$ be an element in $\mathcal{A}_{\ell}^{\infty}$. Since $\alpha_{1}$ and $p_{0} \otimes a_{m n} \in \operatorname{Dom}\left(\delta_{\ell}\right)$ it follows that each of the terms in the infinite sum is an element in $\operatorname{Dom}\left(\delta_{\ell}\right)$. Hence in order to show $a \in \operatorname{Dom}\left(\delta_{\ell}\right)$, it is enough to show that the sum

$$
\sum_{m, n} \delta_{\ell}\left(\alpha_{1}^{* m}\left(p_{0} \otimes a_{m n}\right) \alpha_{1}^{n}\right)+\sum_{m \geq 0} \lambda_{m} \delta_{\ell}\left(\alpha_{1}^{m}\right)+\sum_{n>0} \lambda_{-n} \delta_{\ell}\left(\alpha_{1}^{* n}\right)
$$

converges. Observe that $\delta_{\ell}\left(\alpha_{1}^{* m}\right)=m \alpha_{1}^{* m}, \delta_{\ell}\left(\alpha_{1}^{n}\right)=-n \alpha_{1}^{n}$ and $\delta_{\ell}\left(\alpha_{1}^{* m}\left(p_{0} \otimes a_{m n}\right) \alpha_{1}^{n}\right)=(m-n) \alpha_{1}^{* m}\left(p_{0} \otimes a_{m n}\right) \alpha_{1}^{n}+\alpha_{1}^{* m}\left(p_{0} \otimes \delta_{\ell-1}\left(a_{m n}\right)\right) \alpha_{1}^{n}$.

Since $\delta_{\ell-1}$ is continuous, it follows that $\left\|\delta_{\ell-1}\left(a_{m n}\right)\right\|$ is rapidly decreasing, where $\|\cdot\|$ is the operator norm. (Note that for $b \in \mathscr{B}_{\ell}$, one has $\|b\| \leq\|b\|_{0}$.) Hence the infinite sum

$$
\sum_{m, n} \delta_{\ell}\left(\alpha_{1}^{* m}\left(p_{0} \otimes a_{m n}\right) \alpha_{1}^{n}\right)+\sum_{m \geq 0} \lambda_{m} \delta_{\ell}\left(\alpha_{1}^{m}\right)+\sum_{n>0} \lambda_{-n} \delta_{\ell}\left(\alpha_{1}^{* n}\right)
$$


converges absolutely in the operator norm. Therefore $a \in \operatorname{Dom}\left(\delta_{\ell}\right)$. Since $\delta_{\ell-1}$ is continuous for every $r$ there exists $p$ and $C_{p}$ such that $\left\|\delta_{\ell-1}\left(a^{\prime}\right)\right\|_{r} \leq C_{p}\left\|a^{\prime}\right\|_{p}$. Write $\delta_{\ell-1}\left(a_{m n}\right)$ as $\delta_{\ell-1}\left(a_{m n}\right)=a_{m n}^{\prime}+a_{m n}^{\prime \prime} F_{\ell}+R_{m n}$. Let

$$
\begin{aligned}
A_{0} & =\sum_{m, n} \alpha_{1}^{* m}\left(p_{0} \otimes\left((m-n) a_{m n}+a_{m n}^{\prime}\right)\right) \alpha_{1}^{n}+\sum_{m \geq 0} m \lambda_{m} \alpha_{1}^{m}+\sum_{n>0}(-n) \lambda_{-n} \alpha_{1}^{* n}, \\
A_{1} & =\sum_{m, n} \alpha_{1}^{* m}\left(p_{0} \otimes a_{m n}^{\prime \prime}\right) \alpha_{1}^{n}, \\
R & =\sum_{m, n} \alpha_{1}^{* m}\left(p_{0} \otimes R_{m n}\right) \alpha_{1}^{n} .
\end{aligned}
$$

Then $\delta_{\ell}(a)=A_{0}+A_{1} F_{\ell}+R$. In every seminorm of $\mathcal{A}_{\ell-1}^{\infty}$ the double sequence $\left(a_{m n}^{\prime}\right)$ and $\left(a_{m n}^{\prime \prime}\right)$ are rapidly decreasing. Also $R_{m n}$ is rapidly decreasing in every seminorm of $\mathrm{OP}_{D_{\ell}}^{-\infty}$. Hence $A_{0}, A_{1} \in \mathcal{A}_{\ell}^{\infty}$ and as in the proof of (2), it follows that $R$ is smoothing and given $r, s$ there exists $t$ and $C_{t}$ such that $\|R\|_{r, s} \leq C_{t}\|a\|_{t}$. Fix an $r \geq 0$ and choose $t>1+r$ and $C_{t}>1$ such that $\left\|\delta_{\ell-1}\left(a^{\prime}\right)\right\|_{r} \leq C_{t}\left\|a^{\prime}\right\|_{t}$ for every $a^{\prime} \in \mathcal{A}_{\ell-1}^{\infty}$. Now $\left\|A_{0}\right\|_{r} \leq C_{t}\|a\|_{t}$ and $\left\|A_{1}\right\|_{r} \leq C_{t}\|a\|_{t}$. This shows that the map $a \rightarrow \delta_{\ell}(a) \in \mathscr{B}_{\ell}$ is continuous. Since $\left[D_{\ell}, b\right]=\delta_{\ell}(b) F_{\ell}+\left|D_{\ell}\right|\left[F_{\ell}, b\right]$, the second part of (3) follows as $\left[F_{\ell}, b\right]$ is smoothing by (2). This proves (3).

Part (4) follows from (2) and (3).

We next prove a lemma that will be crucial in the computation of the dimension spectrum. For an $r$ tuple $n=\left(n_{1}, n_{2}, \ldots, n_{r}\right) \in \mathbb{N}^{r}$, we will write $|n|$ for $\sum_{i=1}^{r} n_{i}$. For $r=0$, we let $\mathbb{N}^{0}=\{0\}$.

Lemma 3.7. Let $r \geq 0$ and $s \geq 1$ be integers. Let $(a(n))_{n \in \mathbb{N}^{r}}$ be rapidly decreasing. Then the function

$$
\xi(z):=\sum_{\substack{n \in \mathbb{N}^{r}, m \in \mathbb{N}^{s} \\|n|+|m| \geq 1}} \frac{a(n)}{(|n|+|m|)^{z}}
$$

is meromorphic with simple poles in $\{1,2, \ldots, s\}$ and $\operatorname{Res}_{z=s} \xi(z)=\frac{1}{(s-1) !} \sum_{n} a(n)$.

Proof. First observe that for $\operatorname{Re} z>r+s$,

$$
\begin{aligned}
\xi(z) & =\sum_{N \geq 1} \frac{1}{N^{z}}\left(\sum_{|n|+|m|=N} a(n)\right) \\
& =\sum_{N \geq 1} \frac{1}{N^{z}}\left(\sum_{|n| \leq N} a(n) \sum_{m:|m|=N-|n|} 1\right) \\
& =\sum_{N \geq 1} \frac{1}{N^{z}}\left(\sum_{|n| \leq N} a(n)\left(\begin{array}{c}
N-|n|+s-1 \\
s-1
\end{array}\right)\right) .
\end{aligned}
$$

Note that for a function $(b(n))_{n \in \mathbb{N}^{r}}$ of rapid decay, the sequence $\left(\sum_{|n| \geq N} b(n)\right)_{N \in \mathbb{N}}$ is of rapid decay. Now $\left(\begin{array}{c}N-|n|+s-1 \\ s-1\end{array}\right)=\sum_{k=0}^{s-1} g_{k}(n) N^{k}$, where $g_{k}(n)$ is a polynomial 
in $\left(n_{1}, n_{2}, \ldots, n_{r}\right)$ and $g_{s-1}(n)=\frac{1}{(s-1) !}$. Hence modulo a holomorphic function $\xi(z)=\sum_{k=0}^{s-1}\left(\sum_{n} g_{k}(n) a(n)\right) \zeta(z-k)$. Now the result follows from the fact that $\zeta(z)$ is meromorphic with a simple pole at $z=1$ with residue 1 .

We will next prove that the spectral triple $\left(\mathcal{A}_{\ell}^{\infty}, \mathscr{H}_{\ell}, D_{\ell}\right)$ is regular and has discrete dimension spectrum with simple poles at $\{1,2, \ldots, \ell+1\}$.

Remark 3.8. Recall that the unitaries $U_{w}$ for $w=\left(w_{1}, w_{2}, \ldots, w_{\ell+1}\right) \in \mathbb{T}^{\ell+1}$ are given by $U_{w} e_{\gamma}=w_{1}^{\gamma_{1}} w_{2}^{\gamma_{2}} \ldots w_{\ell+1}^{\gamma_{\ell+1}} e_{\gamma}$. A bounded operator $T$ on $\mathscr{H}_{\ell}$ is said to be homogeneous of degree $\left(m_{1}, m_{2}, \ldots, m_{\ell+1}\right)$ if $U_{w} T U_{w}^{*}=w_{1}^{m_{1}} w_{2}^{m_{2}} \ldots w_{\ell+1}^{m_{\ell+1}} T$. If $T$ is homogeneous of degree $\left(m_{1}, m_{2}, \ldots, m_{\ell+1}\right) \neq(0, \ldots, 0)$ then $\operatorname{Trace}\left(T\left|D_{\ell}\right|^{-z}\right)=$ 0 if $\operatorname{Re}(z)>\ell+1$ since $U_{w}$ 's commute with the operator $\left|D_{\ell}\right|$.

Proposition 3.9. The spectral triple $\left(\mathcal{A}_{\ell}^{\infty}, \mathscr{H}_{\ell}, D_{\ell}\right)$ is regular. It has $\{1,2, \ldots, \ell+1\}$ as the dimension spectrum with only simple poles.

Proof. Regularity of the spectral triple follows from Proposition 3.6. We now prove that for $b \in \mathcal{B}_{\ell}$, the function $\operatorname{Trace}\left(b\left|D_{\ell}\right|^{-z}\right)$ is meromorphic with simple poles at $\{1,2, \ldots, \ell+1\}$. Since Trace $\left(b\left|D_{\ell}\right|^{-z}\right)$ is holomorphic for $b \in \mathrm{OP}^{-\infty}$, we need only to show that for $a \in \mathcal{A}_{\ell}^{\infty}$, the functions $\operatorname{Trace}\left(a\left|D_{\ell}\right|^{-z}\right)$ and $\operatorname{Trace}\left(a F_{\ell}\left|D_{\ell}\right|^{-z}\right)$ extend to meromorphic functions with simple poles at $\{1,2, \ldots, \ell+1\}$. Now any element $a \in \mathcal{A}_{\ell}^{\infty}$ can be written as $a=a^{0}+a^{1}$ where $a^{0}$ is homogeneous of degree 0 and $a^{1}$ is an infinite sum of homogeneous elements of non zero degrees. Hence, by Remark 3.8, $\operatorname{Trace}\left(a\left|D_{\ell}\right|^{-z}\right)=\operatorname{Trace}\left(a^{0}\left|D_{\ell}\right|^{-z}\right)$ and $\operatorname{Trace}\left(a F_{\ell}\left|D_{\ell}\right|^{-z}\right)=\operatorname{Trace}\left(a^{0} F_{\ell}\left|D_{\ell}\right|^{-z}\right)$. Thus it is enough to consider the functions $\operatorname{Trace}\left(a\left|D_{\ell}\right|^{-z}\right)$ and $\operatorname{Trace}\left(a F_{\ell}\left|D_{\ell}\right|^{-z}\right)$ where $a$ is homogeneous of degree 0 .

It is easy to see that the set of homogeneous elements of degree 0 in $\mathcal{A}_{\ell}^{\infty}$ is

$$
\left\{\sum_{i=0}^{\ell}\left(\sum_{n \in \mathbb{N}^{i}} \lambda_{n}^{i}\left(p_{n_{1}} \otimes p_{n_{2}} \otimes \ldots p_{n_{i}} \otimes 1\right)\right):\left(\lambda_{n}^{i}\right) \text { is of rapid decay for all } i\right\},
$$

where $p_{k}=S^{* k} p_{0} S^{k}$. Let $a=\sum_{i=0}^{\ell}\left(\sum_{n} \lambda_{n}^{i}\left(p_{n_{1}} \otimes p_{n_{2}} \otimes \ldots p_{n_{i}} \otimes 1\right)\right.$ be a homogeneous element of degree 0 in $C^{\infty}\left(S_{q}^{2 \ell+1}\right)$. Then

$$
\operatorname{Trace}\left(a\left|D_{\ell}\right|^{-z}\right)=2 \sum_{i=0}^{\ell} \sum_{\substack{n \in \mathbb{N}^{i}, t \in \mathbb{N} \\ m \in \mathbb{N}^{\ell-i}}} \frac{\lambda_{n}^{i}}{(|n|+|m|+t)^{z}}+\sum_{i=0}^{\ell} \sum_{\substack{n \in \mathbb{N}^{i} \\ m \in \mathbb{N}^{\ell-i}}} \frac{\lambda_{n}^{i}}{(|n|+|m|)^{z}}
$$

Now $\sum_{n \in \mathbb{N}^{\ell}} \frac{\lambda_{n}^{\ell}}{(|n|)^{z}}$ is holomorphic and hence, modulo a holomorphic function,

$$
\operatorname{Trace}\left(a\left|D_{\ell}\right|^{-z}\right)=2 \sum_{i=0}^{\ell}\left(\sum_{\substack{n \in \mathbb{N}^{i}, t \in \mathbb{N} \\ m \in \mathbb{N}^{\ell-i}}} \frac{\lambda_{n}^{i}}{(|n|+|m|+t)^{z}}\right)+\sum_{i=0}^{\ell-1}\left(\sum_{\substack{n \in \mathbb{N}^{i} \\ m \in \mathbb{N}^{\ell-i}}} \frac{\lambda_{n}^{i}}{(|n|+|m|)^{z}}\right) .
$$


It follows from Lemma 3.7 that $\operatorname{Trace}\left(a\left|D_{\ell}\right|^{-z}\right)$ is meromorphic with simple poles in the set $\{1,2, \ldots, \ell+1\}$. Similarly one can show that $\operatorname{Trace}\left(a F_{\ell}\left|D_{\ell}\right|^{-z}\right)$ is meromorphic with simple poles in $\{1,2, \ldots, \ell\}$. Fix $0 \leq i \leq \ell$. Let $\left(\lambda_{n}\right)_{n \in \mathbb{N}^{i}}$ be such that $\sum_{n} \lambda_{n}=1$. Let $a=\sum_{n \in \mathbb{N}^{i}} \lambda_{n}\left(p_{n_{1}} \otimes p_{n_{2}} \otimes p_{n_{i}} \otimes 1\right)$. Then one has $\operatorname{Res}_{z=\ell+1-i} \operatorname{Trace}\left(a\left|D_{\ell}\right|^{-z}\right)=\frac{2}{(\ell-i) !}$ by Lemma 3.7 and by the above equation. Hence every $k \in\{1,2, \ldots, \ell+1\}$ is in the dimension spectrum. This completes the proof.

\section{4. $\mathrm{SU}_{q}(\ell+1)$-equivariant spectral triple}

4.1. Left multiplication operators. Let us recall from [4] some basic facts on representations of $C\left(\mathrm{SU}_{q}(\ell+1)\right)$ on $L_{2}\left(\mathrm{SU}_{q}(\ell+1)\right)$ by left multiplication. Irreducible unitary representations of the quantum group $\mathrm{SU}_{q}(\ell+1)$ are indexed by Young tableaux $\lambda=\left(\lambda_{1}, \ldots, \lambda_{\ell+1}\right)$ where $\lambda_{i} \in \mathbb{N}$ and $\lambda_{1} \geq \lambda_{2} \geq \cdots \geq \lambda_{\ell+1}=0$ (Theorem $1.5,[15])$. Denote by $u^{\lambda}$ the irreducible unitary indexed by $\lambda$. Basis elements of the Hilbert space $\mathscr{H}_{\lambda}$ on which $u^{\lambda}$ acts can be parametrized by arrays of the form

$$
\boldsymbol{r}=\left(\begin{array}{ccccc}
r_{11} & r_{12} & \ldots & r_{1, \ell} & r_{1, \ell+1} \\
r_{21} & r_{22} & \ldots & r_{2, \ell} & \\
& \ldots & & & \\
r_{\ell, 1} & r_{\ell, 2} & & & \\
r_{\ell+1,1} & & & &
\end{array}\right),
$$

where the $r_{i j}$ 's are integers satisfying $r_{1 j}=\lambda_{j}$ for $j=1, \ldots, \ell+1, r_{i j} \geq r_{i+1, j} \geq$ $r_{i, j+1} \geq 0$ for all $i, j$, and the top row coincides with $\lambda$. This is known as GelfandTsetlin tableau, briefly GT tableau. Let $\{e(\lambda, r): \boldsymbol{r}$ is a GT tableau with top row $\lambda\}$ be an orthonormal basis for $\mathscr{H}_{\lambda}$. Denote the matrix entries of $u^{\lambda}$ with respect to this basis by $u_{\boldsymbol{r}, \boldsymbol{s}}^{\lambda}$. Note that the generators $u_{i j}$ of the $\mathrm{C}^{*}$-algebra $C\left(\mathrm{SU}_{q}(\ell+1)\right)$ are the matrix entries of the irreducible $\mathbb{1}=(1,0, \ldots, 0)$. The collection $\left\{u_{\boldsymbol{r}, \boldsymbol{s}}^{\lambda}: \lambda, \boldsymbol{r}, \boldsymbol{s}\right\}$ forms a complete orthogonal set of vectors in $L_{2}\left(\mathrm{SU}_{q}(\ell+1)\right)$. Denote by $e_{\boldsymbol{r}, \boldsymbol{s}}^{\lambda}$, or by $e_{\boldsymbol{r}, \boldsymbol{s}}$ for short (as $\boldsymbol{r}$ and $\boldsymbol{s}$ specify $\lambda$ ), the normalized $u_{\boldsymbol{r}, \boldsymbol{s}}^{\lambda}$, s, i.e., $e_{\boldsymbol{r}, \boldsymbol{s}}=\left\|u_{\boldsymbol{r}, \boldsymbol{s}}^{\lambda}\right\|^{-1} u_{\boldsymbol{r}, \boldsymbol{s}}^{\lambda}$. Then $\left\{e_{\boldsymbol{r}, s}: \boldsymbol{r}, \boldsymbol{s}\right\}$ forms a complete orthonormal basis for $L_{2}\left(\mathrm{SU}_{q}(\ell+1)\right)$.

Let $\rho$ be the half-sum of positive roots of $\operatorname{sl}(\ell+1)$ and $\lambda(\boldsymbol{r})$ be the weight of the weight vector $e(\lambda, \boldsymbol{r})$. Let $F_{\lambda}$ be the unique intertwiner in $\operatorname{Mor}\left(u^{\lambda},\left(u^{\lambda}\right)^{c c}\right)$ with Trace $F_{\lambda}=$ Trace $F_{\lambda}^{-1}$ (here, for a representation $u$, its contragradient representation is denoted by $u^{c}$; see [11] for details). Then one has $\left\|u_{\boldsymbol{r} \boldsymbol{s}}^{\lambda}\right\|=d_{\lambda}^{-\frac{1}{2}} q^{-\psi(\boldsymbol{r})}$, where

$$
\begin{aligned}
\psi(\boldsymbol{r}) & =(\rho, \lambda(\boldsymbol{r}))=-\frac{\ell}{2} \sum_{j=1}^{\ell+1} r_{1 j}+\sum_{i=2}^{\ell+1} \sum_{j=1}^{\ell+2-i} r_{i j}, \\
d_{\lambda} & =\text { Trace } F_{\lambda}=\sum_{\boldsymbol{r}: \boldsymbol{r}_{1}=\lambda} q^{2 \psi(\boldsymbol{r})} .
\end{aligned}
$$


Write

$$
\kappa(\boldsymbol{r}, \boldsymbol{m})=d_{\lambda}^{\frac{1}{2}} d_{\mu}^{-\frac{1}{2}} q^{\psi(\boldsymbol{r})-\psi(\boldsymbol{m})} .
$$

From equation (4.19) in [4], we have

$$
\pi\left(u_{i j}\right) e_{\boldsymbol{r} \boldsymbol{s}}^{\lambda}=\sum_{\mu, \boldsymbol{m}, \boldsymbol{n}} C_{q}(\mathbb{1}, \lambda, \mu ; i, \boldsymbol{r}, \boldsymbol{m}) C_{q}(\mathbb{1}, \lambda, \mu ; j, \boldsymbol{s}, \boldsymbol{n}) \kappa(\boldsymbol{r}, \boldsymbol{m}) e_{\boldsymbol{m} \boldsymbol{n}}^{\mu},
$$

where $C_{q}$ denote the Clebsch-Gordon coefficients.

For our subsequent analysis, we will compute the quantities $C_{q}(i, \boldsymbol{r}, \boldsymbol{s})$ and $\kappa(\boldsymbol{r}, \boldsymbol{m})$ appearing in the above formula. We will use the formulae given in ([11], p. 220), keeping in mind that for our case (i.e. for $\mathrm{SU}_{q}(\ell+1)$ ), the top right entry of the GT tableau is zero.

For a positive integer $j$ with $1 \leq j \leq \ell+1$, let

$$
\mathbb{M}_{j}:=\left\{\left(m_{1}, m_{2}, \ldots, m_{j}\right) \in \mathbb{N}^{j}: 1 \leq m_{i} \leq \ell+2-i \text { for } 1 \leq i \leq j\right\} .
$$

For $M=\left(m_{1}, m_{2}, \ldots, m_{i}\right) \in \mathbb{M}_{i}$, denote by $M(\boldsymbol{r})$ the tableau $\boldsymbol{s}$ defined by

$$
s_{j k}= \begin{cases}r_{j k}+1 & \text { if } k=m_{j}, 1 \leq j \leq i, \\ r_{j k} & \text { otherwise. }\end{cases}
$$

With this notation, observe now that $C_{q}(i, \boldsymbol{r}, \boldsymbol{s})$ will be zero unless $\boldsymbol{s}$ is $M(\boldsymbol{r})$ for some $M \in \mathrm{M}_{i}$. (One has to keep in mind, however, that not every tableau of the form $M(\boldsymbol{r})$ is a valid GT tableau.)

From ([11], p. 220), we have

$$
C_{q}(i, \boldsymbol{r}, M(\boldsymbol{r}))=\prod_{a=1}^{i-1} R\left(\boldsymbol{r}, a, m_{a}, m_{a+1}\right) \times R^{\prime}\left(\boldsymbol{r}, i, m_{i}\right),
$$

where

$$
\begin{aligned}
& R(\boldsymbol{r}, a, j, k)=\operatorname{sgn}(k-j) q^{\frac{1}{2}\left(-r_{a j}+r_{a+1, k}-k+j\right)}\left(\prod_{\substack{i=1 \\
i \neq j}}^{\ell+2-a} \frac{\left[r_{a, i}-r_{a+1, k}-i+k\right]_{q}}{\left[r_{a, i}-r_{a, j}-i+j\right]_{q}}\right. \\
& \left.\prod_{\substack{i=1 \\
i \neq k}}^{\ell+1-a} \frac{\left[r_{a+1, i}-r_{a, j}-i+j-1\right]_{q}}{\left[r_{a+1, i}-r_{a+1, k}-i+k-1\right]_{q}}\right)^{\frac{1}{2}} \\
& \left.R^{\prime}(\boldsymbol{r}, a, j)=q^{\frac{1}{2}\left(1-j+\sum_{i=1}^{\ell+1-a}\right.} r_{a+1, i}-\sum_{\substack{i=1 \\
i \neq j}}^{\ell+2-a} r_{a, i}\right) \\
& \cdot\left(\frac{\prod_{i=1}^{\ell+1-a}\left[r_{a+1, i}-r_{a j}-i+j-1\right]_{q}}{\prod_{\substack{\ell=1 \\
i \neq j}}^{\ell+2-a}\left[r_{a, i}-r_{a j}-i+j\right]_{q}}\right)^{\frac{1}{2}}
\end{aligned}
$$

where, for an integer $n,[n]_{q}$ denotes the $q$-number $\left(q^{n}-q^{-n}\right) /\left(q-q^{-1}\right)$ and $\operatorname{sgn}(k-j)$ is 1 if $k \geq j$ and is -1 if $k<j$. 
Remark 4.1. Let us look at the denominators in the above expressions. The integers $r_{a, i}-r_{a, j}$ and $j-i$ are of the same sign. Therefore for $i \neq j$, the quantity $r_{a, i}-r_{a, j}-i+j$ is nonzero. Similarly $r_{a+1, i}-r_{a+1, k}$ and $k-i$ are of the same sign. So if $i \neq k$, then $r_{a+1, i}-r_{a+1, k}-i+k-1$ can be zero only when $r_{a+1, i}=r_{a+1, k}$ and $k=i+1$. Now if $\boldsymbol{r}$ and $M(\boldsymbol{r})$ are GT tableaux, then $M(\boldsymbol{r})_{a+1, m_{a+1}}=$ $r_{a+1, m_{a+1}}+1$ and $M(\boldsymbol{r})_{a+1, i}=r_{a+1, i}$ for $i \neq m_{a+1}$. Therefore if $m_{a+1}=$ $i+1$, then $r_{a+1, i}-\left(r_{a+1, m_{a+1}}+1\right) \geq 0$, i.e., $r_{a+1, i}-r_{a+1, m_{a+1}} \geq 1$. Hence $r_{a+1, i}-r_{a+1, m_{a+1}}-i+m_{a+1}-1 \geq 1$. In other words, all the $q$-numbers appearing in the denominator in eq. (4.6) are nonzero. Thus no problem arises from division by zero.

Remark 4.2. This is essentially a repetition of Remark 4.1 of [4]. The formulae (4.7) and (4.8) are obtained from eqs. (45) and (46), p. 220, [11] by replacing $q$ with $q^{-1}$. Equation (45) is a special case of the more general formula (48), p. 221, [11]. However, there is a small error in eq. (48) there. The correct form can be found in eqs. (3.1), (3.2a), (3.2b) in [1]. Here we have incorporated that correction in eqs. (4.7) and (4.8).

We next compute the quantities $R(\boldsymbol{r}, a, j, k)$ and $R^{\prime}(\boldsymbol{r}, a, j)$.

For a positive integer $n$, denote by $Q(n)$ the number $\left(1-q^{2 n}\right)^{1 / 2}$. Then for any two integers $m$ and $n$, one has

$$
\left|\frac{[m]_{q}}{[n]_{q}}\right|=q^{-|m|+|n|}\left(\frac{Q(|m|)}{Q(|n|)}\right)^{2} .
$$

The next two lemmas are obtained from eqs. (4.7) and (4.8) using the above equality repeatedly and the fact that $r_{a, i} \geq r_{a+1, i} \geq r_{a, i+1}$ for all $a$ and $i$.

Lemma 4.3. For a GT tableau $\boldsymbol{r}=\left(r_{a b}\right)$, denote by $H_{a b}(\boldsymbol{r})$ and $V_{a b}(\boldsymbol{r})$ the following differences: $H_{a b}(\boldsymbol{r}):=r_{a+1, b}-r_{a, b+1}$ and $V_{a b}(\boldsymbol{r}):=r_{a b}-r_{a+1, b}$. Then one has

$$
R(\boldsymbol{r}, a, j, k)=\operatorname{sgn}(k-j) q^{P(\boldsymbol{r}, a, j, k)+S(\boldsymbol{r}, a, j, k)} L(\boldsymbol{r}, a, j, k),
$$

where

$$
\begin{aligned}
P(\boldsymbol{r}, a, j, k)= & \sum_{j \wedge k \leq i<j \vee k} H_{a i}(\boldsymbol{r})+2 \sum_{k<i<j} V_{a i}(\boldsymbol{r}), \\
S(\boldsymbol{r}, a, j, k)= & \begin{cases}2(j-k-1)+1 & \text { if } j>k, \\
0 & \text { if } j \leq k,\end{cases} \\
L(\boldsymbol{r}, a, j, k)= & \prod_{\substack{i=1 \\
i \neq j}}^{\ell+2-a} \frac{Q\left(\left|r_{a, i}-r_{a+1, k}-i+k\right|\right)}{Q\left(\left|r_{a, i}-r_{a, j}-i+j\right|\right)} \\
& \cdot \prod_{\substack{i=1 \\
i \neq k}}^{\ell+1-a} \frac{Q\left(\left|r_{a+1, i}-r_{a, j}-i+j-1\right|\right)}{Q\left(\left|r_{a+1, i}-r_{a+1, k}-i+k-1\right|\right)} .
\end{aligned}
$$


Lemma 4.4. One has

$$
R^{\prime}(\boldsymbol{r}, a, j)=q^{P^{\prime}(\boldsymbol{r}, a, j)} L^{\prime}(\boldsymbol{r}, a, j)
$$

where

$$
\begin{aligned}
P^{\prime}(\boldsymbol{r}, a, j) & =\sum_{j \leq i<\ell+2-a} H_{a i}(\boldsymbol{r}), \\
L^{\prime}(\boldsymbol{r}, a, j) & =\frac{\prod_{i=1}^{\ell+1-a} Q\left(\left|r_{a+1, i}-r_{a, j}-i+j-1\right|\right)}{\prod_{\substack{\ell+2-a \\
i=1 \\
i \neq j}}^{\ell \neq} Q\left(\left|r_{a, i}-r_{a, j}-i+j\right|\right)} .
\end{aligned}
$$

Combining Lemmas 4.3 and 4.4, we get the following expression for the CG coefficient $C_{q}(i, \boldsymbol{r}, M)$.

Lemma 4.5. For a move $M \in M_{i}$, let $\operatorname{sgn}(M)$ denote the product $\prod_{a=1}^{i-1} \operatorname{sgn}\left(m_{a+1}-\right.$ $\left.m_{a}\right)$. Then one has

$$
C_{q}(i, \boldsymbol{r}, M)=\operatorname{sgn}(M) q^{B(M)+C(\boldsymbol{r}, M)}\left(\prod_{a=1}^{i-1} L\left(\boldsymbol{r}, a, m_{a}, m_{a+1}\right)\right) L^{\prime}\left(\boldsymbol{r}, i, m_{i}\right),
$$

where

$$
\begin{aligned}
B(M)= & \sum_{j: m_{j}>m_{j+1}}\left(2\left(m_{j}-m_{j+1}-1\right)+1\right), \\
C(\boldsymbol{r}, M)= & \sum_{a=1}^{i-1}\left(\sum_{m_{a} \wedge m_{a+1} \leq b<m_{a} \vee m_{a+1}} H_{a b}(\boldsymbol{r})+2 \sum_{m_{a+1}<b<m_{a}} V_{a b}(\boldsymbol{r})\right) \\
& +\sum_{m_{i} \leq b<\ell+2-i} H_{i b}(\boldsymbol{r})
\end{aligned}
$$

Lemma 4.6. $\left(\prod_{a=1}^{i-1} L\left(\boldsymbol{r}, a, m_{a}, m_{a+1}\right)\right) L^{\prime}\left(\boldsymbol{r}, i, m_{i}\right)=1+o(q)$.

Proof. This is a consequence of the following two inequalities:

$$
\left|1-(1-x)^{\frac{1}{2}}\right|<x \quad \text { for } 0 \leq x \leq 1,
$$

and, for $0<r<1$,

$$
\left|1-(1-x)^{-\frac{1}{2}}\right|<c x \text { for } 0 \leq x \leq r,
$$

where $c$ is some fixed constant that depends on $r$.

Next we come to the computation of $\kappa(\boldsymbol{r}, \boldsymbol{m})$. Since $C_{q}(i, \boldsymbol{r}, \boldsymbol{m})$ is 0 unless $\boldsymbol{m}$ is of the form $M(\boldsymbol{r})$ for some move $M=\left(m_{1}, \ldots, m_{i}\right)$, we need only to compute $\kappa(\boldsymbol{r}, M(\boldsymbol{r}))$, which we denote by $\kappa(\boldsymbol{r}, M)$. 
Since

$$
\psi(s)=-\frac{\ell}{2} \sum_{j=1}^{\ell+1} s_{1 j}+\sum_{i=2}^{\ell+1} \sum_{j=1}^{\ell+2-i} s_{i j}
$$

we have

$$
\begin{aligned}
& q^{\psi(\boldsymbol{r})-} \boldsymbol{\psi}(M(\boldsymbol{r})) \\
& \quad=q^{-\frac{\ell}{2} \sum_{j=1}^{\ell+1} r_{1 j}+\sum_{i=2}^{\ell+1} \sum_{j=1}^{\ell+2-i} r_{i j}+\frac{\ell}{2}\left(\sum_{j=1}^{\ell+1} r_{1 j}+1\right)-\left(\sum_{i=2}^{\ell+1} \sum_{j=1}^{\ell+2-i} r_{i j}+i-1\right)} \\
& \quad=q^{\frac{\ell}{2}-i+1} .
\end{aligned}
$$

Let $\lambda=\left(\lambda_{1}, \ldots, \lambda_{\ell}, 0\right)$ be the top row of $\boldsymbol{r}$. Then

$$
\min \left\{\psi(s): s_{1}=\lambda\right\}=-\frac{\ell}{2} \sum_{1}^{\ell} \lambda_{i}+\sum_{k=2}^{\ell}(k-1) \lambda_{k} .
$$

Hence

$$
d_{\lambda}=\sum_{s: s_{1}=\lambda} q^{2 \psi(s)}=q^{-\ell \sum_{1}^{\ell} \lambda_{i}+2 \sum_{k=2}^{\ell}(k-1) \lambda_{k}}\left(1+q^{2} \phi\left(q^{2}\right)\right),
$$

where $\phi$ is a polynomial. Therefore

$$
d_{\lambda}=q^{-\ell \sum_{1}^{\ell} \lambda_{i}+2 \sum_{k=2}^{\ell}(k-1) \lambda_{k}}(1+o(q)) .
$$

It follows that

$$
\left(\frac{d_{\lambda}}{d_{\lambda+e_{m_{1}}}}\right)^{\frac{1}{2}}=q^{\frac{\ell}{2}-m_{1}+1}(1+o(q))
$$

Thus

$$
\kappa(\boldsymbol{r}, M(\boldsymbol{r}))=q^{\ell+2-i-m_{1}}(1+o(q)) .
$$

Next, observe that

$$
\begin{aligned}
& B(M)+\ell+2-i-m_{1} \\
& =\sum_{j: m_{j}>m_{j+1}}\left(2\left(m_{j}-m_{j+1}-1\right)+1\right)-\left(m_{1}-m_{i}\right)+\ell+2-i-m_{i} \\
& =2 \sum_{j: m_{j}>m_{j+1}}\left(m_{j}-m_{j+1}\right)-\sum_{j: m_{j}>m_{j+1}} 1-\sum_{j=1}^{i-1}\left(m_{j}-m_{j+1}\right)+\ell+2-i-m_{i} \\
& =2 \sum_{j: m_{j}>m_{j+1}}\left(m_{j}-m_{j+1}\right)-\sum_{j=1}^{i-1}\left(m_{j}-m_{j+1}\right)-\sum_{j: m_{j}>m_{j+1}} 1+\ell+2-i-m_{i} \\
& =\sum_{j=1}^{i-1}\left|m_{j}-m_{j+1}\right|-\#\left\{1 \leq j \leq i-1: m_{j}>m_{j+1}\right\}+\ell+2-i-m_{i} .
\end{aligned}
$$


Thus if we write

$$
\begin{aligned}
& A(M)=\sum_{j=1}^{i-1}\left|m_{j}-m_{j+1}\right|-\#\left\{1 \leq j \leq i-1: m_{j}>m_{j+1}\right\}, \\
& K(M)=\ell+2-i-m_{i},
\end{aligned}
$$

then both $A(M)$ and $K(M)$ are nonnegative and $B(M)+\ell+2-i-m_{1}=A(M)+$ $K(M)$. Thus we have

$$
\begin{gathered}
\pi\left(u_{i j}\right) e_{\boldsymbol{r} s}^{\lambda}=\sum_{\substack{M \in \mathbb{M}_{i} \\
M^{\prime} \in \mathbb{M}_{j}}} C_{q}(i, \boldsymbol{r}, M(\boldsymbol{r})) \kappa(\boldsymbol{r}, M) C_{q}\left(j, s, M^{\prime}(\boldsymbol{s})\right) e_{M(\boldsymbol{r}), M^{\prime}(\boldsymbol{s})} \\
=\sum_{\substack{M \in \mathbb{M}_{i} \\
M^{\prime} \in \mathbb{M}_{j}}} \operatorname{sgn}(M) \operatorname{sgn}\left(M^{\prime}\right) q^{A(M)+K(M)+C(\boldsymbol{r}, M)+B\left(M^{\prime}\right)+C\left(\boldsymbol{s}, M^{\prime}\right)} \\
\cdot(1+o(q)) e_{M(\boldsymbol{r}), M^{\prime}(\boldsymbol{s}) .}
\end{gathered}
$$

4.2. The spectral triple. Let us briefly recall from [4] the description of the $L_{2^{-}}$ space of the sphere sitting inside $L_{2}\left(\mathrm{SU}_{q}(\ell+1)\right)$. Let $u^{\mathbb{1}}$ denote the fundamental unitary for $\mathrm{SU}_{q}(\ell+1)$, i. e. the irreducible unitary representation corresponding to the Young tableaux $\mathbb{1}=(1,0, \ldots, 0)$. Similarly write $v^{\mathbb{1}}$ for the fundamental unitary for $\mathrm{SU}_{q}(\ell)$. Fix some bases for the corresponding representation spaces. Then $C\left(\mathrm{SU}_{q}(\ell+1)\right)$ is the $\mathrm{C}^{*}$-algebra generated by the matrix entries $\left\{u_{i j}^{\mathbb{1}}\right\}$ and $C\left(\mathrm{SU}_{q}(\ell)\right)$ is the $\mathrm{C}^{*}$-algebra generated by the matrix entries $\left\{v_{i j}^{\mathbb{\pi}}\right\}$. Now define $\phi$ by

$$
\phi\left(u_{i j}^{\mathbb{1}}\right)= \begin{cases}I & \text { if } i=j=1, \\ v_{i-1, j-1}^{\mathbb{1} 1} & \text { if } 2 \leq i, j \leq \ell+1, \\ 0 & \text { otherwise. }\end{cases}
$$

Then $C\left(\mathrm{SU}_{q}(\ell+1) \backslash \mathrm{SU}_{q}(\ell)\right)$ is the $\mathrm{C}^{*}$-subalgebra of $C\left(\mathrm{SU}_{q}(\ell+1)\right)$ generated by the entries $u_{1, j}$ for $1 \leq j \leq \ell+1$. Define $\psi: C\left(S_{q}^{2 \ell+1}\right) \rightarrow C\left(\mathrm{SU}_{q}(\ell+1) \backslash \mathrm{SU}_{q}(\ell)\right)$ by

$$
\psi\left(z_{i}\right)=q^{-i+1} u_{1, i}^{*} .
$$

This gives an isomorphism between $C\left(\mathrm{SU}_{q}(\ell+1) \backslash \mathrm{SU}_{q}(\ell)\right)$ and $C\left(S_{q}^{2 \ell+1}\right)$ and the diagram

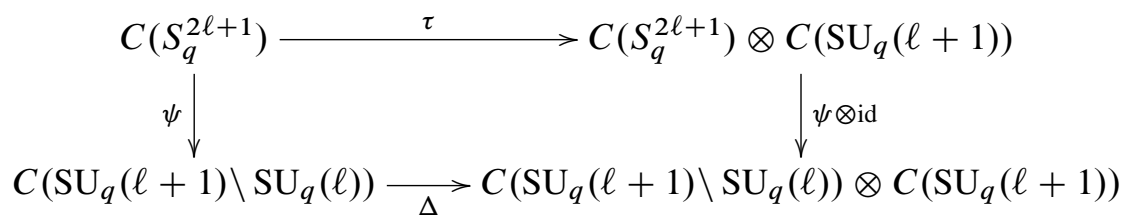

commutes, where $\tau: C\left(S_{q}^{2 \ell+1}\right) \rightarrow C\left(S_{q}^{2 \ell+1}\right) \otimes C\left(\mathrm{SU}_{q}(\ell+1)\right)$ is the homomorphism given by $\tau\left(z_{i}\right)=\sum_{k} z_{k} \otimes u_{k, i}^{*}$ that gives an action of the quantum group 
$\mathrm{SU}_{q}(\ell+1)$ on $S_{q}^{2 \ell+1}$. In other words, $\left(C\left(S_{q}^{2 \ell+1}\right), \mathrm{SU}_{q}(\ell+1), \tau\right)$ is the quotient space $\mathrm{SU}_{q}(\ell+1) \backslash \mathrm{SU}_{q}(\ell)$. This choice of $\psi$ makes $L_{2}\left(\mathrm{SU}_{q}(\ell+1) \backslash \mathrm{SU}_{q}(\ell)\right)$ a span of certain rows of the $e_{\boldsymbol{r}, \boldsymbol{s}}$ 's, as the following two propositions say.

Proposition 4.7 ([4]). Assume that $\ell>1$. The right regular representation $u$ of $G$ keeps the subspace $L_{2}\left(\mathrm{SU}_{q}(\ell+1) \backslash \mathrm{SU}_{q}(\ell)\right)$ invariant, and the restriction of $u$ to $L_{2}\left(\mathrm{SU}_{q}(\ell+1) \backslash \mathrm{SU}_{q}(\ell)\right)$ decomposes as a direct sum of exactly one copy of each of the irreducibles given by the Young tableaux $\lambda_{n, k}:=(n+k, k, k, \ldots, k, 0)$, with $n, k \in \mathbb{N}$.

Proposition 4.8 ([4]). Let $\boldsymbol{r}^{n k}$ denote the GT tableaux given by

$$
r_{i j}^{n k}= \begin{cases}n+k & \text { if } i=j=1, \\ 0 & \text { if } i=1, j=\ell+1, \\ k & \text { otherwise, }\end{cases}
$$

where $n, k \in \mathbb{N}$. Let $\mathcal{G}_{0}^{n, k}$ be the set of all GT tableaux with top row $(n+k, k, \ldots, k, 0)$. Then the family of vectors

$$
\left\{e_{\boldsymbol{r}^{n k, \boldsymbol{s}}}: n, k \in \mathbb{N}, \boldsymbol{s} \in \mathcal{G}_{0}^{n, k}\right\}
$$

forms a complete orthonormal basis for $L_{2}\left(\mathrm{SU}_{q}(\ell+1) \backslash \mathrm{SU}_{q}(\ell)\right)$.

We will denote $\bigcup_{n, k} \mathcal{G}_{0}^{n, k}$ by $\mathcal{G}_{0}$. Since the top row of $\boldsymbol{r}^{n k}$ determines $\boldsymbol{r}^{n k}$ completely and for $e_{\boldsymbol{r}}^{n k}{ }_{, s}$ the top row of $\boldsymbol{s}$ equals the top row of $\boldsymbol{r}^{n k}$, one can index the orthonormal basis $e_{\boldsymbol{r}^{n k}, s}$ just by $s \in \mathcal{G}_{0}$. It was shown in [4] that the restriction of the left multiplication to $C\left(\mathrm{SU}_{q}(\ell+1) \backslash \mathrm{SU}_{q}(\ell)\right) \cong C\left(S_{q}^{2 \ell+1}\right)$ keeps invariant $L_{2}\left(\mathrm{SU}_{q}(\ell+1) \backslash \mathrm{SU}_{q}(\ell)\right) \cong L_{2}\left(S_{q}^{2 \ell+1}\right)$. We will continue to denote this restriction by $\pi$. The operators $\pi\left(z_{j}\right)=q^{-j+1} \pi\left(u_{1, j}^{*}\right)$ will be denoted by $Z_{j, q}$. The $\mathrm{C}^{*}$-algebra $\pi\left(C\left(S_{q}^{2 \ell+1}\right)\right)$ will be denoted by $C_{\ell}$.

The following theorem gives a generic equivariant spectral triple for the spheres $S_{q}^{2 \ell+1}$ constructed in [4].

Theorem 4.9 ([4]). Let $D_{e q}$ be the operator on $L_{2}\left(S_{q}^{2 \ell+1}\right)$ given by

$$
D_{e q} e_{\boldsymbol{r}^{n k}{ }_{, \boldsymbol{s}}}= \begin{cases}k e_{\boldsymbol{r}^{n k}{ }_{, \boldsymbol{s}}} & \text { if } n=0, \\ -(n+k) e_{\boldsymbol{r}^{n k}, \boldsymbol{s}} & \text { if } n>0 .\end{cases}
$$

Then $\left(\mathcal{A}\left(S_{q}^{2 \ell+1}\right), L_{2}\left(S_{q}^{2 \ell+1}\right), D_{e q}\right)$ is an equivariant nondegenerate $(2 \ell+1)$-summable odd spectral triple.

Our main aim in the rest of the paper is to precisely formulate the smooth function algebra for this spectral triple, to establish its regularity, and to compute the dimension spectrum. 
4.3. The case $\boldsymbol{q}=\mathbf{0}$. The $L_{2}$-spaces $L_{2}\left(S_{q}^{2 \ell+1}\right)$ for different values of $q$ can be identified by considering the elements of their canonical orthonormal bases which are parametrized by the same set. Thus we will assume we are working with one single Hilbert space $\mathscr{H}$ with orthonormal basis given by $e_{\boldsymbol{r}^{n, k}}$, where $\boldsymbol{r}^{n, k}$ is as defined earlier and $s$ is given by

$$
\boldsymbol{s}=\left(\begin{array}{ccccccc}
c_{1}=n+k & k & k & \ldots & k & k & d_{1}=0 \\
c_{2} & k & k & \ldots & k & d_{2} & \\
\ldots & & \ldots & & & & \\
c_{\ell-1} & k & d_{\ell-1} & & & & \\
c_{\ell} & d_{\ell} & & & & & \\
c_{\ell+1}=d_{\ell+1} & & & & &
\end{array}\right),
$$

where $c_{1} \geq c_{2} \geq \cdots \geq c_{\ell} \geq k, d_{1} \leq d_{2} \leq \cdots \leq d_{\ell} \leq k$ and $d_{\ell} \leq d_{\ell+1} \leq c_{\ell}$. Since specifying the GT tableau $\boldsymbol{s}$ determines $\boldsymbol{r}^{n, k}$ as well and thus completely specifies the basis element $e_{\boldsymbol{r}^{n, k_{\boldsymbol{s}}}}$, we will sometimes use just $\boldsymbol{s}$ in place of the basis element

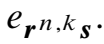

Let us denote by $M_{j}^{ \pm}$the following subsets of $M_{j}$ :

$$
\begin{aligned}
& \mathbb{M}_{j}^{+}=\left\{\left(m_{1}, \ldots, m_{j}\right) \in \mathbb{M}_{j}: m_{i} \in\{1, \ell+2-i\} \text { for } 1 \leq i \leq j, m_{1}=1\right\}, \\
& \mathbb{M}_{j}^{-}=\left\{\left(m_{1}, \ldots, m_{j}\right) \in \mathbb{M}_{j}: m_{i} \in\{1, \ell+2-i\} \text { for } 1 \leq i \leq j, m_{1}=\ell+1\right\} .
\end{aligned}
$$

Let us denote by $N_{i, j}$ the following element of $\mathrm{M}_{j}$ :

$$
N_{i, j}=(\underbrace{1, \ldots, 1}_{i}, \ell+1-i, \ell-i, \ldots, \ell+2-j), \quad 0 \leq i \leq j \leq \ell+1 .
$$

We will denote $N_{i, \ell+1}$ by just $N_{i}$. Then from (4.22), we get

$$
\begin{aligned}
\pi\left(u_{1 j}\right) e_{\boldsymbol{r}^{n, k} \boldsymbol{s}}= & \sum_{M \in \mathbb{M}_{j}^{+}} \operatorname{sgn}(M) q^{\ell+k+B(M)+C(\boldsymbol{s}, M)}(1+o(q)) e_{\boldsymbol{r}^{n+1, k}, M(s)} \\
& +\sum_{M \in \mathbb{M}_{j}^{-}} \operatorname{sgn}(M) q^{B(M)+C(\boldsymbol{s}, M)}(1+o(q)) e_{\boldsymbol{r}^{n, k-1}, M(\boldsymbol{s})} .
\end{aligned}
$$

Therefore

$$
\begin{aligned}
Z_{j, q}^{*} e_{\boldsymbol{r}^{n, k} \boldsymbol{s}}= & \sum_{M \in \mathbb{M}_{j}^{+}} \operatorname{sgn}(M) q^{-j+1+\ell+k+B(M)+C(\boldsymbol{s}, M)}(1+o(q)) e_{\boldsymbol{r}^{n+1, k}, M(s)} \\
& +\sum_{M \in \mathbb{M}_{j}^{-}} \operatorname{sgn}(M) q^{-j+1+B(M)+C(\boldsymbol{s}, M)}(1+o(q)) e_{\boldsymbol{r}^{n, k-1}, M(s)} .
\end{aligned}
$$

Let us first look at the cases $1 \leq j \leq \ell$. Then the power of $q$ in the first summation is positive. Therefore none of the terms would survive for $q=0$. For terms in the 
second summation, assume that $M \in \mathbb{M}_{j}$ with $m_{1}=\ell+1$ and $m_{i}=1$ for some $i \leq j$. Let $a=\min \left\{2 \leq i \leq j: m_{i}=1\right\}$. Then $m_{i}=\ell+2-i$ for $1 \leq i \leq a-1$ so that

$$
\begin{aligned}
B(M) & \geq \sum_{i=1}^{a-2}(2((\ell+2-i)-(\ell+1-i)-1)+1)+2(\ell+3-a-1-1)+1 \\
& =a-2+2(\ell-a+1)+1 \\
& =2 \ell-a+1 .
\end{aligned}
$$

Hence $B(M)+1-j>0$ and so such terms will not survive for $q=0$. Therefore, the only term that will survive is the one corresponding to $M=N_{0, j}=$ $(\ell+1, \ell, \ell-1, \ldots, \ell+2-j)$. In this case we have $B(M)=j-1, C(s, M)=d_{j}$ and $\operatorname{sgn}(M)=(-1)^{j-1}$. Therefore

$$
Z_{j, 0}^{*} e_{\boldsymbol{r}^{n, k} \boldsymbol{s}}= \begin{cases}(-1)^{j-1} e_{\boldsymbol{r}^{n, k-1}, N_{0, j}(s)} & \text { if } d_{j}=0 \\ 0 & \text { if } d_{j}>0\end{cases}
$$

Next let us look at the case $j=\ell+1$. Here the first sum will be over all $M$ with $m_{1}=1=m_{\ell+1}$. If $m_{i} \neq 1$ for some $i$, then $B(M)>0$ and therefore the power of $q$ will be positive, so that the term will not survive for $q=0$. If $m_{i}=1$ for all $i$, i.e., if $M=N_{\ell}$, then we have $B(M)=0=C(s, M)$ and $\operatorname{sgn}(M)=1$. Therefore for $q=0$, the first summation will become $e_{\boldsymbol{r}^{n+1, k}, N_{\ell}(s)}$ provided that $k=0$.

The second sum is over all $M$ with $m_{1}=\ell+1$. Let $a=\min \{2 \leq i \leq$ $\left.\ell+1: m_{i}=1\right\}$. Then, as before, $B(M) \geq 2 \ell-a+1$. Therefore if $a \leq \ell$, then $-\ell+B(M) \geq \ell-a+1>0$, so that the term will not survive for $q=0$. If $a=\ell+1$, i.e., if $M=N_{0}$, then $B(M)=\ell, C(s, M)=d_{\ell+1}$ and $\operatorname{sgn}(M)=(-1)^{\ell}$. So, for $q=0$, the second summation will become $(-1)^{\ell} e_{\boldsymbol{r}^{n, k-1}, N_{0}(s)}$ if $k>0$ and $d_{\ell+1}=0$. Thus we have

$$
Z_{\ell+1,0}^{*} e_{\boldsymbol{r}^{n, k}}= \begin{cases}e_{\boldsymbol{r}^{n+1, k}, N_{\ell}(s)} & \text { if } k=0, \\ (-1)^{\ell} e_{\boldsymbol{r}^{n, k-1}, N_{0}(\boldsymbol{s})} & \text { if } k>0, d_{\ell+1}=0, \\ 0 & \text { if } k>0, d_{\ell+1}>0\end{cases}
$$

Next we will establish a natural unitary map between $L_{2}\left(S_{q}^{2 \ell+1}\right)$ and

$$
\mathscr{H}_{\Sigma} \equiv \underbrace{\ell_{2}(\mathbb{N}) \otimes \cdots \otimes \ell_{2}(\mathbb{N})}_{\ell \text { copies }} \otimes \ell_{2}(\mathbb{Z}) \otimes \underbrace{\ell_{2}(\mathbb{N}) \otimes \cdots \otimes \ell_{2}(\mathbb{N})}_{\ell \text { copies }} .
$$

For $t \in \mathbb{R}$, let $t_{+}$denote the positive part $\max \{t, 0\}$ and let $t_{-}$denote the negative part 
$\max \{(-t), 0\}$ of $t$. Let us now observe that for any $\gamma \in \Gamma_{\Sigma}$, the tableau

$$
s(\gamma):=\left(\begin{array}{cccc}
\sum_{1}^{2 \ell+1}\left|\gamma_{i}\right| & \sum_{1}^{\ell} \gamma_{i}+\left(\gamma_{\ell+1}\right)_{+} & \ldots & \sum_{1}^{\ell} \gamma_{i}+\left(\gamma_{\ell+1}\right)_{+} 0 \\
\sum_{1}^{2 \ell}\left|\gamma_{i}\right| & \sum_{1}^{\ell} \gamma_{i}+\left(\gamma_{\ell+1}\right)_{+} & \ldots & \gamma_{1} \\
\ldots & \ldots & & \\
\sum_{1}^{\ell+3}\left|\gamma_{i}\right| & \sum_{1}^{\ell} \gamma_{i}+\left(\gamma_{\ell+1}\right)_{+} \sum_{1}^{\ell-2} \gamma_{i} & \\
\sum_{1}^{\ell+2}\left|\gamma_{i}\right| & \sum_{1}^{\ell-1} \gamma_{i} & & \\
\sum_{1}^{\ell} \gamma_{i}+\left(\gamma_{\ell+1}\right)_{-} & & &
\end{array}\right) .
$$

is in $\mathcal{G}_{0}$. Conversely, let $\boldsymbol{s} \in \mathcal{G}_{0}^{n, k}$ for some $n, k \in \mathbb{N}$ so that $e_{\boldsymbol{r}^{n, k} \boldsymbol{s}}$ is a basis element of $L_{2}\left(S_{q}^{2 \ell+1}\right)$. Note that $s$ is of the form (4.25). Define $\gamma \in \Gamma_{\Sigma}$ as follows:

(1) if $k>d_{\ell+1}$, then

$$
\begin{aligned}
& \gamma_{i}=d_{i+1}-d_{i} \quad \text { for } 1 \leq i \leq \ell-1, \\
& \gamma_{i}=c_{2 \ell+2-i}-c_{2 \ell+3-i} \quad \text { for } \ell+3 \leq i \leq 2 \ell+1, \\
& \gamma_{\ell}=d_{\ell+1}-d_{\ell}, \quad \gamma_{\ell+1}=k-d_{\ell+1}, \quad \gamma_{\ell+2}=c_{\ell}-k,
\end{aligned}
$$

(2) if $k \leq d_{\ell+1}$, then

$$
\begin{aligned}
& \gamma_{i}=d_{i+1}-d_{i} \quad \text { for } 1 \leq i \leq \ell-1, \\
& \gamma_{i}=c_{2 \ell+2-i}-c_{2 \ell+3-i} \quad \text { for } \ell+3 \leq i \leq 2 \ell+1, \\
& \gamma_{\ell}=k-d_{\ell}, \quad \gamma_{\ell+1}=k-d_{\ell+1}, \quad \gamma_{\ell+2}=c_{\ell}-d_{\ell+1} .
\end{aligned}
$$

Then $s(\gamma)=s$. Thus we have a bijective correspondence between $\mathcal{G}_{0}$ and $\Gamma_{\Sigma}$. We will often denote a basis element $e_{\boldsymbol{r}}^{n, k_{\boldsymbol{s}}}$ by $\xi_{\gamma}$ using this bijective correspondence.

Lemma 4.10. Let $\gamma \in \Gamma_{\Sigma}$. For $n \in \mathbb{Z}$, let

$$
Z_{\ell+1,0}^{(n)}:= \begin{cases}Z_{\ell+1,0}^{n} & \text { if } n \geq 0 \\ \left(Z_{\ell+1,0}^{*}\right)^{-n} & \text { if } n<0\end{cases}
$$

Define

$$
\xi_{\gamma}^{\prime}:=Z_{1,0}^{\gamma_{1}} \ldots Z_{\ell, 0}^{\gamma_{\ell}} Z_{\ell+1,0}^{\left(\gamma_{\ell+1}\right)}\left(\begin{array}{ccccc}
\sum_{\ell+2}^{2 \ell+1} \gamma_{i} & 0 & \ldots & 0 & 0 \\
\sum_{\ell+2}^{2 \ell} \gamma_{i} & 0 & \ldots & 0 & \\
\gamma_{\ell+2} & 0 & & & \\
0 & & &
\end{array}\right)
$$

Then $\left\{\xi_{\gamma}^{\prime}: \gamma \in \Gamma_{\Sigma}\right\}$ is an orthonormal basis for $L_{2}\left(S_{q}^{2 \ell+1}\right)$.

Proof. It follows from eqs. (4.28) and (4.29) that the actions of $Z_{j, 0}$ for $1 \leq j \leq \ell$ 
on the basis elements $e_{\boldsymbol{r}}^{n, k} \boldsymbol{s}$ are as follows:

$$
\begin{aligned}
& Z_{j, 0}:\left(\begin{array}{cccccc}
n+k & k & & \ldots & k & k \\
c_{2} & k & & \ldots & k & 0 \\
\ldots & & \ldots & & & \\
c_{j} & k & \ldots & k & 0 & \\
c_{j+1} & k & \ldots & d_{j+1} & & \\
\ldots & & \ldots & & & \\
c_{\ell} & d_{\ell} & & &
\end{array}\right) \longrightarrow \\
& (-1)^{j-1}\left(\begin{array}{cccccc}
1+n+k & 1+k & & \ldots & 1+k & 1+k \\
1+c_{2} & 1+k & & \ldots & 1+k & 0 \\
\ldots & & \ldots & & & \\
1+c_{j} & 1+k & \ldots & 1+k & 0 & \\
1+c_{j+1} & 1+k & \ldots & 1+d_{j+1} & \\
\ldots & & \ldots & & & \\
1+c_{\ell} & 1+d_{\ell} & & & & \\
1+d_{\ell+1} & & & &
\end{array}\right)
\end{aligned}
$$

and is 0 for $\boldsymbol{s}$ with $d_{j}>0$.

Similarly the action of $Z_{\ell+1,0}$ on the basis elements are as follows:

$$
\left(\begin{array}{cccccc}
n & 0 & & \ldots & 0 & 0 \\
c_{2} & 0 & & \ldots & 0 & \\
\ldots & & \ldots & & & \\
c_{\ell-1} & 0 & 0 & & & \\
c_{\ell} & 0 & & & & \\
d_{\ell+1} & & & &
\end{array}\right) \longrightarrow\left(\begin{array}{cccccc}
n-1 & 0 & & \ldots & 0 & 0 \\
c_{2}-1 & 0 & & \ldots & 0 & \\
\ldots & & \ldots & & & \\
c_{\ell-1}-1 & 0 & 0 & & & \\
c_{\ell}-1 & 0 & & & & \\
d_{\ell+1}-1 & & & &
\end{array}\right)
$$

if $d_{\ell+1}>0$, and

$$
\left(\begin{array}{ccccc}
n+k & k & & \ldots & k \\
c_{2} & k & & \ldots & 0 \\
\ldots & & \ldots & & \\
c_{\ell-1} & k & 0 & & \\
c_{\ell} & 0 & & &
\end{array}\right) \longrightarrow(-1)^{\ell}\left(\begin{array}{ccccc}
1+n+k & 1+k & \ldots & 1+k \\
1+c_{2} & 1+k & \ldots & 0 \\
\ldots & & \ldots & & \\
0 & & &
\end{array}\right)
$$

if $d_{\ell+1}=0$. Similarly the action of $Z_{\ell+1,0}^{*}$ on the basis elements are as follows:

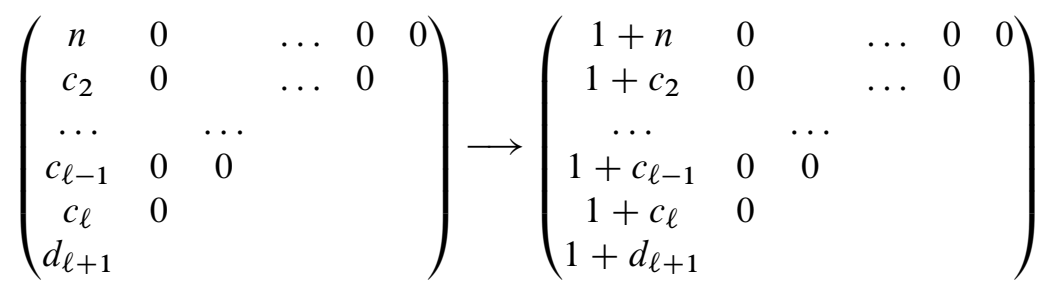


and, for $k>0$,

$$
\left(\begin{array}{ccccc}
n+k & k & \ldots & k 0 \\
c_{2} & k & \ldots & 0 \\
\ldots & & \ldots & \\
c_{\ell-1} & k & 0 & & \\
c_{\ell} & 0 & & & \\
0 & & &
\end{array}\right) \longrightarrow(-1)^{\ell}\left(\begin{array}{ccccc}
n+k-1 & k-1 & \ldots k-1 & 0 \\
c_{2}-1 & k-1 & \ldots & 0 \\
\ldots & & \ldots & \\
c_{\ell-1}-1 & k-1 & 0 & \\
c_{\ell}-1 & 0 & & \\
0 & & &
\end{array}\right)
$$

Then it follows from the above that

$$
\begin{aligned}
& Z_{1,0}^{\gamma_{1}} \ldots Z_{\ell, 0}^{\gamma_{\ell}} Z_{\ell+1,0}^{\left(\gamma_{\ell+1}\right)}\left(\begin{array}{ccccc}
\sum_{\ell+2}^{2 \ell+1} \gamma_{i} & 0 & \ldots & 0 & 0 \\
\sum_{\ell+2}^{2 \ell} \gamma_{i} & 0 & \ldots & 0 & \\
& \ldots & & \\
\gamma_{\ell+2} & 0 & & & \\
0 & & &
\end{array}\right) \\
& =(-1)^{\eta(\gamma)}\left(\begin{array}{cccc}
\sum_{1}^{2 \ell+1}\left|\gamma_{i}\right| & \sum_{1}^{\ell} \gamma_{i}+\left(\gamma_{\ell+1}\right)_{+} & \ldots & \sum_{1}^{\ell} \gamma_{i}+\left(\gamma_{\ell+1}\right)_{+} 0 \\
\sum_{1}^{2 \ell}\left|\gamma_{i}\right| & \sum_{1}^{\ell} \gamma_{i}+\left(\gamma_{\ell+1}\right)_{+} & \ldots & \gamma_{1} \\
\sum_{1}^{\ell+3}\left|\gamma_{i}\right| & \sum_{1}^{\ell} \gamma_{i}+\left(\gamma_{\ell+1}\right)_{+} & \sum_{1}^{\ell-2} \gamma_{i} & \\
\sum_{1}^{\ell+2}\left|\gamma_{i}\right| & \sum_{1}^{\ell-1} \gamma_{i} & & \\
\sum_{1}^{\ell} \gamma_{i}+\left(\gamma_{\ell+1}\right)_{-} & & &
\end{array}\right) \text {, }
\end{aligned}
$$

where $\eta(\gamma):=\sum_{i=1}^{\ell}(i-1) \gamma_{i}+\ell\left(\gamma_{\ell+1}\right)_{+}$. Thus $\xi_{\gamma}^{\prime}=(-1)^{\eta(\gamma)} \xi_{\gamma}$. Therefore it follows that $\left\{\xi_{\gamma}^{\prime}: \gamma \in \Gamma_{\Sigma}\right\}$ is an orthonormal basis for $L_{2}\left(S_{q}^{2 \ell+1}\right)$.

The map $U: L_{2}\left(S_{q}^{2 \ell+1}\right) \rightarrow \mathscr{H}_{\Sigma}$ given by $U \xi_{\gamma}^{\prime}=e_{\gamma}$ sets up a unitary isomorphism between $L_{2}\left(S_{q}^{2 \ell+1}\right)$ and $\mathscr{H}_{\Sigma}$. Let $P$ denote the projection onto the span of $e_{0} \otimes \cdots \otimes e_{0}$ in $\ell_{2}\left(\mathbb{N}^{\ell}\right)$. Then we have

$$
U Z_{j, 0} U^{*}=Y_{j, 0} \otimes I=Y_{j, 0} \otimes P+Y_{j, 0} \otimes(I-P),
$$

and

$$
U D_{e q} U^{*}=D_{\ell} \otimes P-\left|D_{\ell}\right| \otimes(I-P)-I \otimes \tilde{N},
$$

where $\tilde{N}$ is the operator $e_{m_{1}} \otimes \cdots \otimes e_{m_{\ell}} \mapsto\left(\sum m_{i}\right) e_{m_{1}} \otimes \cdots \otimes e_{m_{\ell}}$. In other words, with respect to the decomposition

$$
\mathscr{H}_{\Sigma}=\mathscr{H}_{\ell} \oplus\left(\mathscr{H}_{\ell} \otimes \ell_{2}\left(\mathbb{N}^{\ell} \backslash\{0, \ldots, 0\}\right)\right),
$$

one has

$$
U Z_{j, 0} U^{*}=Y_{j, 0} \oplus\left(Y_{j, 0} \otimes I\right),
$$

and

$$
U D_{e q} U^{*}=D_{\ell} \oplus\left(-\left|D_{\ell}\right| \otimes I-I \otimes \tilde{N}\right) .
$$


Next we will define the smooth function algebra $C_{e q}^{\infty}\left(S_{0}^{2 \ell+1}\right)$ and prove that the spectral triple $\left(C_{e q}^{\infty}\left(S_{0}^{2 \ell+1}\right), \mathcal{H}, D_{e q}\right)$ is regular with simple dimension spectrum $\{1,2, \ldots, 2 \ell+1\}$.

It follows from decomposition (4.31) that if we identify $L_{2}\left(S_{q}^{2 \ell+1}\right)$ with $\mathscr{H}_{\Sigma}$, then the $\mathrm{C}^{*}$-algebra generated by the $Z_{j, 0}$ 's is $A_{\ell} \otimes I$, where $A_{\ell}$ is the $\mathrm{C}^{*}$-algebra generated by the $Y_{j}$ 's in $\mathscr{L}\left(\mathscr{H}_{\ell}\right)$. Therefore it is natural to define

$$
C_{e q}^{\infty}\left(S_{0}^{2 \ell+1}\right)=\left\{a \otimes I: a \in \mathcal{A}_{\ell}^{\infty}\right\} .
$$

Theorem 4.11. The triple $\left(C_{e q}^{\infty}\left(S_{0}^{2 \ell+1}\right), \mathscr{H}_{\Sigma}, D_{e q}\right)$ is a regular spectral triple with simple dimension spectrum $\{1,2, \ldots, 2 \ell+1\}$.

Proof. Since $\mathcal{A}_{\ell}^{\infty}$ is closed under holomorphic function calculus in $A_{\ell}$, it follows that $C_{e q}^{\infty}\left(S_{0}^{2 \ell+1}\right)$ is closed under holomorphic function calculus in $C^{*}\left(\left\{Z_{j, 0}: 1 \leq\right.\right.$ $j \leq \ell+1\})=A_{\ell} \otimes I$. In order to show regularity, let us introduce the algebra

$$
\mathcal{B}_{e q}:=\left\{a \otimes P+b \otimes(I-P): a, b \in \mathscr{B}_{\ell}\right\}
$$

Clearly $\mathscr{B}_{e q}$ contains $C_{e q}^{\infty}\left(S_{0}^{2 \ell+1}\right)$. We will show that $\mathscr{B}_{e q}$ is closed under derivations with both $\left|D_{e q}\right|$ as well as $D_{e q}$. This will prove regularity of the spectral triple $\left(C_{e q}^{\infty}\left(S_{0}^{2 \ell+1}\right), \mathcal{H}, D_{e q}\right)$.

Note that $\left|D_{e q}\right|=\left|D_{\ell}\right| \otimes I+I \otimes \tilde{N}$. Since $I \otimes \tilde{N}$ commutes with every element of $\mathcal{B}_{e q}$, we get $\delta(a \otimes P+b \otimes(I-P))=\left[\left|D_{\ell}\right|, a\right] \otimes P+\left[\left|D_{\ell}\right|, b\right] \otimes(I-P)$ and $\left[D_{e q}, a \otimes P+b \otimes(I-P)\right]=\left[D_{\ell}, a\right] \otimes P-\left[\left|D_{\ell}\right|, b\right] \otimes(I-P)$. Since $B_{\ell}$ is closed under derivations with $\left|D_{\ell}\right|$ and $D_{\ell}$, it follows that $\mathscr{B}_{e q}$ is closed under derivations with $\left|D_{e q}\right|$ and $D_{e q}$.

Next we compute the dimension spectrum of the spectral triple. For $w \in \mathbb{T}^{\ell+1}$, let $\widetilde{U}_{w}:=U_{w} \otimes I$. Then $\left|D_{e q}\right|$ commutes with $\widetilde{U}_{w}$. Hence again it is enough to consider homogeneous elements of degree 0 . Now by Lemma 3.7 it follows that for $b \in \mathscr{B}_{e q}$ with $b$ homogeneous of degree 0 the function $\operatorname{Trace}\left(b\left|D_{e q}\right|^{-z}\right)$ is meromorphic with simple poles and the poles lie in $\{1,2, \ldots, 2 \ell+1\}$. To show that every point of $\{1,2, \ldots, 2 \ell+1\}$ is in the dimension spectrum, observe that

$$
\operatorname{Trace}\left(\left|D_{e q}\right|^{-z}\right)=\Sigma_{k=0}^{2 \ell}\left(2 c_{k}^{2 \ell}-c_{k}^{2 \ell-1}\right) \zeta(z-k),
$$

where $c_{k}^{r}$ is defined as the coefficient of $N^{k}$ in $\left(\begin{array}{c}N+r \\ r\end{array}\right)$. Note that for $0 \leq k \leq r$ one has $c_{k}^{r}>0$. Also note the recurrence $r c_{k}^{r}=c_{k-1}^{r-1}+r c_{k}^{r-1}$. Hence $c_{k}^{r} \geq c_{k}^{r-1}$. Now from eq. (4.35) it follows that $\operatorname{Res}_{z=k+1} \operatorname{Trace}\left(\left|D_{e q}\right|^{-z}\right)=2 c_{k}^{2 \ell}-c_{k}^{2 \ell-1}>0$ for $0 \leq k \leq 2 \ell$. This proves that every point of $\{1,2, \ldots, 2 \ell+1\}$ is in the dimension spectrum, which completes the proof.

We will need the fact that $\operatorname{Trace}\left(\left|D_{e q}\right|^{-z}\right)$ is meromorphic with simple poles at $\{1,2, \ldots, 2 \ell+1\}$ with nonzero residue and hence we state it as a separate lemma. 
Lemma 4.12. The function Trace $\left(\left|D_{e q}\right|^{-z}\right)$ is meromorphic with simple poles at $\{1,2, \ldots, 2 \ell+1\}$. Also the residue $\operatorname{Res}_{z=k} \operatorname{Trace}\left(\left|D_{\text {eq }}\right|^{-z}\right)$ is nonzero for $k \in$ $\{1,2, \ldots, 2 \ell+1\}$.

4.4. Regularity and dimension spectrum for $q \neq 0$. Consider the smooth subalgebra of the Toeplitz algebra defined as

$$
\begin{array}{r}
\mathcal{T}^{\infty}=\left\{\sum_{j, k \in \mathbb{N}} \lambda_{j k} S^{* j} p_{0} S^{k}+\sum_{k \geq 0} \lambda_{k} S^{k}+\sum_{k>0} \lambda_{-k} S^{* k}: \lambda_{j k},\left(\lambda_{k}\right)\right. \text { are } \\
\text { rapidly decreasing }\} .
\end{array}
$$

For $a:=\sum_{j, k \in \mathbb{N}} \lambda_{j k} S^{* j} p_{0} S^{k}+\sum_{k \geq 0} \lambda_{k} S^{k}+\sum_{k>0} \lambda_{-k} S^{* k} \in \mathcal{T}^{\infty}$, define the seminorm $\|\cdot\|_{m}$ by $\|a\|_{m}:=\sum(1+|j|+|k|)^{m}\left|\lambda_{k l}\right|+\sum(1+|k|)^{m}\left|\lambda_{k}\right|$. Equipped with this family of seminorms, $\mathcal{T}^{\infty}$ is a Fréchet algebra. We will denote by $\mathcal{T}_{k}^{\infty}$ the $k$-fold tensor product of $\mathcal{T}^{\infty}$.

Lemma 4.13. The triple $\left(\mathcal{T}^{\infty}, \ell_{2}(\mathbb{N}), N\right)$ is a regular spectral triple. More precisely, $\mathcal{T}^{\infty}$ is contained in $\operatorname{Dom}(\delta)$, where $\delta$ is the unbounded derivation $[N, \cdot]$ and leaves the algebra $\mathcal{T}^{\infty}$ invariant. Also the map $\delta: \mathcal{T}^{\infty} \rightarrow \mathcal{T}^{\infty}$ is continuous.

Proof. Note that $[N, S]=-S$ and $[N, p]=0$. Now the lemma follows from the fact that the unbounded derivation $\delta$ is closed.

For $\alpha \in \mathbb{N}^{2} \cup \mathbb{Z}$, let

$$
W_{\alpha}= \begin{cases}S^{* m} p_{0} S^{n} & \text { if } \alpha=(m, n) \\ S^{r} & \text { if } \alpha=r \geq 0 \\ S^{* r} & \text { if } \alpha=r<0\end{cases}
$$

For $\alpha \in \mathbb{N}^{2} \cup \mathbb{Z}$, define $|\alpha|$ to be $|m|+|n|$ if $\alpha=(m, n) \in \mathbb{N}^{2}$ and the usual absolute value $|\alpha|$ if $\alpha \in \mathbb{Z}$. For an $\ell$ tuple $\alpha=\left(\alpha_{1}, \alpha_{2}, \ldots, \alpha_{\ell}\right)$ in $\left(\mathbb{N}^{2} \cup \mathbb{Z}\right)^{\ell}$, let $|\alpha|=\sum\left|\alpha_{i}\right|$ and $W_{\alpha}:=W_{\alpha_{1}} \otimes W_{\alpha_{2}} \otimes \ldots W_{\alpha_{\ell}}$. We need the following simple lemma whose proof we omit since it is easy to prove.

Lemma 4.14. The natural tensor product representation of $\mathcal{T}_{\ell}^{\infty}$ on $\ell_{2}(\mathbb{N})^{\otimes \ell}$ is injective. Thus we identify $\mathcal{T}_{\ell}^{\infty}$ with its range which is $\left\{\sum x_{\alpha} W_{\alpha}: \sum(1+|\alpha|)^{p}\left|x_{\alpha}\right|<\infty\right.$ for every $p$.

Remark 4.15. The tensor product representation of $\mathrm{OP}_{D_{\ell}}^{-\infty} \otimes \mathcal{T}_{\ell}^{\infty}$ on $\mathscr{L}\left(\mathscr{H}_{\Sigma}\right)$ is injective since $\mathrm{OP}_{D_{\ell}}^{-\infty}:=S\left(\mathscr{H}_{\ell}\right)$, and hence we identify $\mathrm{OP}_{D_{\ell}}^{\ell} \otimes \mathcal{T}_{\ell}^{\infty}$ with its image.

For an operator $T$, let $L_{T}$ denote the left multiplication map $X \mapsto T X$. Then for $T \in \mathrm{OP}_{D_{\ell}}^{0}$, the map $L_{T}: \mathrm{OP}_{D_{\ell}}^{-\infty} \rightarrow \mathrm{OP}_{D_{\ell}}^{-\infty}$ is continuous. Note that if $A$ is a Fréchet algebra and $a \in A$, then $L_{a}$ is a continuous linear operator. 
Lemma 4.16. Let $T \in \mathrm{OP}_{D_{\ell}}^{0}$ and $a \in \mathcal{T}_{\ell}^{\infty}$. Then the map $L_{T \otimes a}$ leaves the algebra $\mathrm{OP}_{D_{\ell}}^{-\infty} \otimes \mathcal{T}_{\ell}^{\infty}$ invariant. Moreover $L_{T \otimes a}=L_{T} \otimes L_{a}$ on the algebra $\mathrm{OP}_{D_{\ell}}^{-\infty} \otimes \mathcal{T}_{\ell}^{\infty}$.

Proof. Clearly $L_{T \otimes a}=L_{T} \otimes L_{a}$ on the algebraic tensor product $\mathrm{OP}_{D_{\ell}}^{-\infty} \otimes_{\mathrm{alg}} \mathcal{T}_{\ell}^{\infty}$. Now let $a \in \mathrm{OP}_{D_{\ell}}^{-\infty} \otimes \mathcal{T}_{\ell}^{\infty}$. Then there exists a sequence $a_{n} \in \mathrm{OP}_{D_{\ell}}^{-\infty} \otimes_{\text {alg }} \mathcal{T}_{\ell}^{\infty}$ which converges to $a$ in $\mathrm{OP}_{D_{\ell}}^{-\infty} \otimes \mathcal{T}_{\ell}^{\infty}$. Also $a_{n}$ converges to $a$ in the operator norm. Now the result follows from the continuity of $L_{T \otimes a}$ and $L_{T} \otimes L_{a}$.

Proposition 4.17. Let

$$
\mathcal{B}:=\mathcal{B}_{e q}+\mathrm{OP}_{D_{\ell}}^{-\infty} \otimes \mathcal{T}_{\ell}^{\infty}
$$

Then one has the following.

(1) The vector space $\mathcal{B}$ is an algebra.

(2) The algebra $\mathcal{B}$ is invariant under the derivations $\delta:=\left[\left|D_{e q}\right|, \cdot\right]$ and $\left[D_{e q}, \cdot\right]$.

(3) For $b \in \mathcal{B}$, the commutator $\left[F_{e q}, b\right] \in \mathrm{OP}_{D_{e q}}^{-\infty}$.

(4) For $b \in \mathcal{B}$, the function $\operatorname{Trace}\left(b\left|D_{e q}\right|^{-z}\right)$ is meromorphic with only simple poles lying in $\{1,2, \ldots, 2 \ell+1\}$.

Proof. Lemma 4.16 and the fact that $\mathscr{B}_{\ell} \subset \mathrm{OP}^{0}$ implies that $\mathcal{B}$ is an algebra. As seen in Theorem 4.11, it follows that $\mathscr{B}_{e q}$ is invariant under $\delta$ and $\left[D_{e q}, \cdot\right]$. Also (3) and (4) hold for $b \in \mathscr{B}_{e q}$. Hence to complete the proof it is enough to consider (2), (3) and (4) for the algebra $\mathrm{OP}_{D_{\ell}}^{-\infty} \otimes \mathcal{T}_{\ell}^{\infty}$.

Lemma 2.6 and the decomposition $\left|D_{e q}\right|=\left|D_{\ell}\right| \otimes 1+1 \otimes \tilde{N}$ implies that $\delta$ leaves the algebra $\mathrm{OP}_{D_{\ell}}^{-\infty} \otimes \mathcal{T}_{\ell}^{\infty}$ invariant. Now note that $P \in \mathrm{OP}_{\tilde{N}}^{-\infty}$, it follows that left and right multiplication by $F_{\ell} \otimes P$ and $1 \otimes P$ sends $\mathrm{OP}_{D_{\ell}}^{-\infty} \otimes \mathcal{T}_{\ell}^{\infty}$ to $\mathrm{OP}_{D_{e q}}^{-\infty} \equiv$ $\mathrm{OP}_{D_{\ell}}^{-\infty} \otimes \mathrm{OP}_{\tilde{N}}^{-\infty}$. Since $F_{e q}=F_{\ell} \otimes P-I \otimes(I-P)$, it follows that $\left[F_{e q}, b\right]$ is smoothing for every $b \in \mathrm{OP}_{D_{\ell}}^{-\infty} \otimes \mathcal{T}^{\infty}$. Now the invariance of $\mathrm{OP}_{D_{\ell}}^{-\infty} \otimes \mathcal{T}_{\ell}^{\infty}$ under $\left[D_{e q}, \cdot\right]$ follows from the equation $\left[D_{e q}, b\right]=\delta(b) F_{e q}+\left|D_{e q}\right|\left[F_{e q}, b\right]$ and the fact that $\mathrm{OP}_{D_{e q}}^{-\infty}:=\mathrm{OP}_{D_{\ell}}^{-\infty} \otimes \mathrm{OP}_{\tilde{N}}^{-\infty}$ is contained in $\mathrm{OP}_{D_{\ell}}^{-\infty} \otimes \mathcal{T}_{\ell}^{\infty}$.

We prove that for $b \in \mathrm{OP}_{D_{\ell}}^{-\infty} \otimes \mathcal{T}_{\ell}^{\infty}$ the function Trace $\left(b\left|D_{e q}\right|^{-z}\right)$ is meromorphic with simple poles and the poles lie in $\{1,2, \ldots, \ell\}$. For $w \in \mathbb{T}^{2 \ell+1}$, let $U_{w}=$ $U_{w_{1}} \otimes U_{w_{2}} \otimes \ldots U_{w_{2 \ell+1}}$ be the unitary operator on $\mathscr{H}_{\Sigma}$. Clearly $U_{w}\left|D_{e q}\right| U_{w}^{*}=\left|D_{e q}\right|$ for $w \in \mathbb{T}^{2 \ell+1}$. Hence it is enough to consider Trace $\left(b\left|D_{e q}\right|^{-z}\right)$ with $b$ homogeneous of degree 0 .

An element $b$ is homogeneous if and only if it commutes with the operators $U_{w}$ for all $w \in \mathbb{T}^{2 \ell+1}$. This implies that $b$ must be of the form $e_{\gamma} \mapsto \phi(\gamma) e_{\gamma}$ for some function $\phi$, i.e., $b=\sum_{\gamma} \phi(\gamma) p_{\gamma}$. An operator of the form $\sum_{\gamma \in \Gamma_{\Sigma_{\ell}}} \phi(\gamma) p_{\gamma}$ is in $\mathrm{OP}_{D_{\ell}}^{-\infty}$ if and only if $\phi(\gamma)$ is rapidly decaying on $\Gamma_{\Sigma_{\ell}}$. Also, using the description of $\mathcal{T}^{\infty}$, it follows that an operator of the form $\sum_{n \in \mathbb{N}} \phi(n) p_{n}$ belongs to $\mathcal{T}^{\infty}$ if and only if $\phi(\cdot)-\lim _{n \rightarrow \infty} \phi(n)$ is rapidly decreasing. Thus combining these, one can see that the operator $\sum_{\gamma} \phi(\gamma) p_{\gamma}$ belongs to $\mathrm{OP}_{D_{\ell}}^{-\infty} \otimes \mathcal{T}_{\ell}^{\infty}$ if and only if $\phi$ is a linear 
combination of $\phi_{A}$ with $A$ varying over subsets of $\Sigma$ containing $\Sigma_{\ell}$, where each $\phi_{A}(\gamma)$ depends only on $\gamma_{A}$ and $\phi_{A}\left(\gamma_{A}\right)$ is rapidly decreasing on $\Gamma_{A}$. For an element $b=\sum_{\gamma} \phi_{A}(\gamma) p_{\gamma}$, one has

$$
\operatorname{Trace}\left(b\left|D_{e q}\right|^{-z}\right)=\sum_{\gamma} \frac{\phi_{A}(\gamma)}{|\gamma|^{z}}=\sum_{\gamma} \frac{\phi_{A}\left(\gamma_{A}\right)}{\left(\left|\gamma_{A}\right|+\left|\gamma_{\Sigma \backslash A}\right|\right)^{z}} .
$$

By Lemma 3.7 it follows that Trace $\left(b\left|D_{e q}\right|^{-z}\right)$ is meromorphic with simple poles lying in $\{1,2, \ldots,|\Sigma \backslash A|\} \subseteq\{1,2, \ldots, \ell\}$. This completes the proof.

4.5. The smooth function algebra $C^{\infty}\left(S_{q}^{2 \ell+1}\right)$. In this section we will define a dense Fréchet $C^{*}$-algebra $C^{\infty}\left(S_{q}^{2 \ell+1}\right)$ of $C_{\ell}=\pi\left(C\left(S_{q}^{2 \ell+1}\right)\right)$ and show that it is closed under holomorphic functional calculus. Let $B_{\ell}$ be the $\mathrm{C}^{*}$-algebra generated by $A_{\ell}$ and $F_{\ell}$. Recall that $\&$ denotes the $\mathrm{C}^{*}$-algebra generated by $C(\mathbb{T})$ and $F_{0}$.

Lemma 4.18. The $C^{*}$-algebra $\mathcal{E}$ contains $\mathcal{K}$ and $\mathscr{E} / \mathcal{K}$ is isomorphic to the $C^{*}$ algebra $C(\mathbb{T}) \oplus C(\mathbb{T})$.

Proof. Let $\left|e_{m}\right\rangle\left\langle e_{n}\right|$ be the matrix units in $\mathcal{K}\left(\ell_{2}(\mathbb{Z})\right)$. Note that $\left[F_{0}, S^{*}\right] S=$ $2\left|e_{0}\right\rangle\left\langle e_{0}\right|$. Hence $p_{0} \equiv\left|e_{0}\right\rangle\left\langle e_{0}\right| \in \mathcal{E}$. Now $S^{* m} p_{0} S^{n}=\left|e_{m}\right\rangle\left\langle e_{n}\right|$. Hence $\mathcal{K} \subset \mathcal{E}$. Let $P_{0}:=\frac{1+F_{0}}{2}$. Then $\left[P_{0}, f\right]$ is compact for every $f$. Thus $\mathcal{E} / \mathcal{K}$ is generated by $C(\mathbb{T})$ and a projection $P_{0}$ which is in the center of $\mathcal{E} / \mathcal{K}$. Now consider the map

$$
C(\mathbb{T}) \oplus C(\mathbb{T}) \ni(f, g) \mapsto f P_{0}+g\left(1-P_{0}\right) \quad(\bmod \mathcal{K}) \in \mathcal{E} / \mathcal{K} .
$$

We claim that this map is an isomorphism. To prove this, we need to show that if $f P_{0}$ is compact then $f=0$, and if $g\left(1-P_{0}\right)$ is compact then $g=0$.

Assume that $f P_{0}$ is compact for $f \in C(\mathbb{T})$. Fix an $r \in \mathbb{Z}$. Since $f P_{0}$ is compact, it follows that $\left|\left\langle f P_{0}\left(e_{n}\right), e_{n+r}\right\rangle\right|=|\hat{f}(r)|$ converges to 0 as $n \rightarrow+\infty$. Hence $\hat{f}(r)=0$ for every $r$. This proves that $f=0$. Similarly one can show that if $g\left(1-P_{0}\right)$ is compact then $g=0$. This completes the proof.

Lemma 4.19. The $C^{*}$-algebra $B_{\ell}$ contains $\mathcal{K}\left(\mathscr{H}_{\ell}\right)$ and the map $(a, b) \mapsto a P_{\ell}+$ $b\left(1-P_{\ell}\right)(\bmod \mathcal{K})$ from $C\left(S_{q}^{2 \ell+1}\right) \oplus C\left(S_{q}^{2 \ell+1}\right)$ to $B_{\ell} / \mathcal{K}\left(\mathcal{H}_{\ell}\right)$ is an isomorphism.

Proof. For $\ell=0$ this is just Lemma 4.18. So let us prove the statement for $\ell \geq 1$. Since $A_{\ell}$ contains $\mathcal{K}\left(\ell_{2}\left(\mathbb{N}^{\ell}\right)\right) \otimes C(\mathbb{T})$, it follows that $B_{\ell}$ contains $\mathcal{K}\left(\mathcal{H}_{\ell}\right)$. Observe that $\left[P_{\ell}, \alpha_{i}\right]=0$ for $1 \leq i \leq \ell$ and $\left[P_{\ell}, \alpha_{\ell+1}\right]$ is compact. Therefore it follows that $\left[P_{\ell}, a\right]$ is compact for every $a \in A_{\ell}$. Hence the map $(a, b) \mapsto a P_{\ell}+b\left(1-P_{\ell}\right)$ $(\bmod \mathcal{K})$ from $A_{\ell} \oplus A_{\ell}$ to $B_{\ell} / \mathcal{K}$ is a *-algebra homomorphism onto $B_{\ell} / \mathcal{K}$. We will show that the map is one-to-one. For that we have to show that if $a P_{\ell}$ is compact with $a \in A_{\ell}$ then $a=0$, and if $b\left(1-P_{\ell}\right)$ is compact with $b \in A_{\ell}$ then $b=0$.

Suppose now that $a P_{\ell}$ is compact. Observe that $B_{\ell} \subset \mathcal{T}_{\ell} \otimes \mathscr{E}$ and $a P_{\ell}=$ $a\left(I \otimes P_{0}\right)$. Since $a P_{\ell}$ is compact, if we apply the symbol map $\sigma$ on the $\ell$-th copy 
of $\mathcal{T}$, we get $\sigma_{\ell}(a) \otimes P_{0}=0$. Hence $a$ is in the ideal $\mathcal{K}\left(\ell_{2}(\mathbb{N})^{\otimes \ell}\right) \otimes C(\mathbb{T})$. For $m, n \in \mathbb{N}^{\ell}$, let $e_{m n}$ be the "matrix" units. Let $a_{m n}=\left(e_{m m} \otimes 1\right) a\left(e_{n n} \otimes 1\right)$. Then $a_{m n}=e_{m n} \otimes f_{m n}$ for some $f_{m n} \in C(\mathbb{T})$. Since $a P_{\ell}$ is compact, it follows that $f_{m n} P_{0}$ is compact as $P_{\ell}=I \otimes P_{0}$ commutes with $e_{n n} \otimes I$. By the case $\ell=0$, it follows that $f_{m n}=0$ and hence $a_{m n}=0$ for every $m, n$. Thus $a=0$. Similarly one can show that if $b\left(1-P_{\ell}\right)$ is compact then $b=0$. This completes the proof.

Let $\mathbb{B}$ be the $\mathrm{C}^{*}$ algebra on $\mathscr{H}_{\Sigma}$ generated by $A_{\ell} \otimes I, P_{\ell} \otimes 1$ and $1 \otimes P$ and $J:=\mathcal{K}\left(\mathscr{H}_{\ell}\right) \otimes \mathcal{T}_{\ell}$. Note that $J$ is an ideal since $\mathbb{B}_{\ell}$ is contained in $\mathcal{T}_{\ell} \otimes \mathcal{E} \otimes \mathcal{T}_{\ell}$. The next proposition identifies the quotient $\mathbb{B} / J$.

Proposition 4.20. Let $\rho: A_{\ell} \oplus A_{\ell} \oplus A_{\ell} \oplus A_{\ell} \rightarrow \mathbb{B} / J$ be the map $\left(a_{1}, a_{2}, a_{3}, a_{4}\right) \mapsto a_{1} P_{\ell} \otimes P+a_{2} P_{\ell} \otimes(1-P)+a_{3}\left(1-P_{\ell}\right) \otimes P+a_{4}\left(1-P_{\ell}\right) \otimes(1-P)$

from $A_{\ell} \oplus A_{\ell} \oplus A_{\ell} \oplus A_{\ell}$ into $\mathbb{B}$ composed with the canonical projection from $\mathbb{B}$ onto $\mathrm{B} / J$. Then $\rho$ is an isomorphism.

Proof. First note that since $\left[P_{\ell}, a\right] \in \mathcal{K}$ for $a \in A_{\ell}$, it follows that $P_{\ell} \otimes I$ and $I \otimes P$ are in the center of $\mathbb{B} / J$. Hence the map $\rho$ is an algebra homomorphism. By the definition of $\mathbb{B}$ it follows that $\rho$ is onto. Thus we have to show $\rho$ is one-to-one.

Suppose that $a=a_{1} P_{\ell} \otimes P+a_{2} P_{\ell} \otimes(1-P)+a_{3}\left(1-P_{\ell}\right) \otimes P+a_{4}\left(1-P_{\ell}\right) \otimes$ $(1-P) \in J$. Let $\epsilon: \mathcal{T} \rightarrow \mathbb{C}$ be the map $e v_{1} \circ \sigma$, where $e v_{1}$ is evaluation at the point 1. Now consider the map id $\otimes \epsilon^{\otimes \ell}: \mathcal{T}_{\ell} \otimes \mathcal{E} \otimes \mathcal{T}_{\ell} \rightarrow \mathcal{T}_{\ell} \otimes \mathcal{E}$. Note that $I \otimes \epsilon^{\otimes \ell}$ sends $J$ to $\mathcal{K}\left(\mathscr{H}_{\ell}\right)$. Hence $\left(I \otimes \epsilon^{\otimes \ell}\right)(a)=a_{2} P_{\ell}+a_{4}\left(1-P_{\ell}\right) \in \mathcal{K}\left(\mathscr{H}_{\ell}\right)$. Hence by Lemma 4.19, it follows that $a_{2}=0=a_{4}$. Since left multiplication by $I \otimes P$ sends the ideal $J$ to $\mathcal{K}\left(\mathscr{H}_{\Sigma}\right)$, it follows that $(I \otimes P) a=a_{1} P_{\ell} \otimes P+a_{3}\left(1-P_{\ell}\right) \otimes P$ is compact. Hence $a_{1} P_{\ell}+a_{3}\left(1-P_{\ell}\right)$ is compact. Thus again by Lemma 4.19, it follows that $a_{1}=0=a_{3}$. This completes the proof.

Now we prove that $\mathcal{B}$ is closed under holomorphic functional calculus in $\mathbb{B}$. Let $\mathcal{J}:=\mathrm{OP}_{D_{\ell}}^{-\infty} \otimes \mathcal{T}_{\ell}^{\infty}$. Note that

$$
\begin{aligned}
& \mathcal{B}:=\left\{a_{1} P_{\ell} \otimes P+a_{2} P_{\ell} \otimes(1-P)+a_{3}\left(1-P_{\ell}\right) \otimes P\right. \\
&\left.+a_{4}\left(1-P_{\ell}\right) \otimes(1-P)+R: a_{1}, a_{2}, a_{3}, a_{4} \in A_{\ell}^{\infty}, R \in \mathcal{J}\right\} .
\end{aligned}
$$

Proposition 4.21. The algebra $\mathcal{B}$ has the following properties:

(1) If $a_{1} P_{\ell} \otimes P+a_{2} P_{\ell} \otimes(1-P)+a_{3}\left(1-P_{\ell}\right) \otimes P+a_{4}\left(1-P_{\ell}\right) \otimes(1-P) \in \mathcal{J}$ then $a_{i}=0$ for $i=1,2,3,4$. Hence $\mathcal{B}$ is isomorphic to the direct sum $A_{\ell}^{\infty} \oplus A_{\ell}^{\infty} \oplus A_{\ell}^{\infty} \oplus A_{\ell}^{\infty} \oplus \mathcal{J}$. Equip $\mathcal{B}$ with the Fréchet space structure coming from this direct sum decomposition.

(2) The algebra $\mathcal{B}$ is a Fréchet $C^{*}$-algebra contained in $\mathbb{B}$. Moreover the inclusion $\mathcal{B} \subset \mathbb{B}$ is continuous. 
(3) The algebra $\mathcal{B}$ is closed under holomorphic functional calculus in $\mathbb{B}$.

Proof. Proposition 4.20 implies (1). Parts (2) and (3) follow from Proposition 3.6. Now by Proposition 4.20 one has the exact sequence

$$
0 \rightarrow J \rightarrow \mathbb{B} \rightarrow A_{\ell} \oplus A_{\ell} \oplus A_{\ell} \oplus A_{\ell} \rightarrow 0
$$

At the smooth algebra level we have the exact sequence

$$
0 \rightarrow \mathcal{J} \rightarrow \mathcal{B} \stackrel{\theta}{\rightarrow} A_{\ell}^{\infty} \oplus A_{\ell}^{\infty} \oplus A_{\ell}^{\infty} \oplus A_{\ell}^{\infty} \rightarrow 0 .
$$

Since $\mathcal{J} \subset J$ and $A_{\ell}^{\infty} \subset A_{\ell}$ are closed under holomorphic functional calculus, it follows from Theorem 3.2, part 2, [13] that $\mathcal{B}$ is spectrally invariant in $\mathbb{B}$. Since by part (2), the Fréchet topology of $\mathcal{B}$ is finer than the norm topology, it follows that $\mathcal{B}$ is closed in the holomorphic function calculus of $\mathbb{B}$.

Remark 4.22. One can prove that $\mathrm{OP}_{D_{\ell}}^{-\infty} \otimes \mathcal{T}_{\ell}^{\infty}$ is closed under holomorphic functional calculus in $\mathcal{K}\left(\mathscr{H}_{\ell}\right) \otimes \mathcal{T}_{\ell}$ in the same manner by applying Theorem 3.2, part 2 , [13] and by using the extension (after tensoring suitably)

$$
0 \rightarrow \mathcal{K} \rightarrow \mathcal{T} \rightarrow C(\mathbb{T}) \rightarrow 0
$$

at the $\mathrm{C}^{*}$-algebra level and the extension

$$
0 \rightarrow S\left(\ell_{2}(\mathbb{N})\right) \rightarrow \mathcal{T}^{\infty} \rightarrow C^{\infty}(\mathbb{T}) \rightarrow 0
$$

at the Fréchet algebra level.

Corollary 4.23. Define the smooth function algebra $C^{\infty}\left(S_{q}^{2 \ell+1}\right)$ by

$$
C^{\infty}\left(S_{q}^{2 \ell+1}\right)=\left\{a \in \mathcal{B} \cap C_{\ell}: \theta(a) \in \iota\left(\mathcal{A}_{\ell}^{\infty}\right)\right\},
$$

where $\theta$ is as in the proof of Proposition 4.21 and $\iota: A_{\ell} \rightarrow A_{\ell} \oplus A_{\ell} \oplus A_{\ell} \oplus A_{\ell}$ is the inclusion map $a \mapsto a \oplus a \oplus a \oplus a$. Then the algebra $C^{\infty}\left(S_{q}^{2 \ell+1}\right)$ is closed in $\mathcal{B}$ and is closed under holomorphic functional calculus in $C_{\ell}$.

Proof. Let $j: \mathcal{B} \rightarrow \mathscr{L}\left(\mathscr{H}_{\Sigma}\right)$ denote the inclusion map. Then, by definition, $C^{\infty}\left(S_{q}^{2 \ell+1}\right)=\theta^{-1}\left(\iota\left(A_{\ell}^{\infty}\right)\right) \cap j^{-1}\left(C_{\ell}\right)$. Since $\theta$ and $j$ are continuous and as $\iota\left(A_{\ell}^{\infty}\right)$ and $C_{\ell}$ are closed, it follows that $C^{\infty}\left(S_{q}^{2 \ell+1}\right)$ is closed in $\mathcal{B}$. Hence $C^{\infty}\left(S_{q}^{2 \ell+1}\right)$ is a Fréchet algebra. Also $C^{\infty}\left(S_{q}^{2 \ell+1}\right)$ is *-closed as $\rho$ is *-preserving. Now let $a \in C^{\infty}\left(S_{q}^{2 \ell+1}\right)$ be invertible in $C_{\ell}$. Then $a$ is invertible in $\mathscr{L}\left(\mathscr{H}_{\Sigma}\right)$. By Proposition 4.21, it follows that $a^{-1} \in \mathcal{B}$. By the closedness of $A_{\ell}^{\infty}$ under holomorphic functional calculus, it follows that $\theta\left(a^{-1}\right) \in \iota\left(A_{\ell}^{\infty}\right)$. Thus one has $a^{-1} \in C^{\infty}\left(S_{q}^{2 \ell+1}\right)$. We have already seen that the Fréchet topology of $\mathcal{B}$ is finer than the norm topology. The same is therefore true for the topology of $C^{\infty}\left(S_{q}^{2 \ell+1}\right)$. Hence it is closed under holomorphic functional calculus in $C_{\ell}$. 
Proposition 4.24. The operators $Z_{j, q}$ belong to $C^{\infty}\left(S_{q}^{2 \ell+1}\right)$. Hence $C^{\infty}\left(S_{q}^{2 \ell+1}\right)$ is a dense subalgebra of $C_{\ell}$ that contains $\pi\left(\mathcal{A}\left(S_{q}^{2 \ell+1}\right)\right)$.

The proof of this proposition will be given in the next subsection.

We are now in a position to prove the main theorem.

Theorem 4.25. The triple $\left(C^{\infty}\left(S_{q}^{2 \ell+1}\right), \mathscr{H}_{\Sigma}, D_{e q}\right)$ is a regular spectral triple with simple dimension spectrum $\{1,2, \ldots, 2 \ell+1\}$.

Proof. Since the inclusion $C^{\infty}\left(S_{q}^{2 \ell+1}\right) \subset \mathcal{B}$ holds, the regularity of the spectral triple $\left(C^{\infty}\left(S_{q}^{2 \ell+1}\right), \mathscr{H}_{\Sigma}, D_{e q}\right)$ follows from the regularity of the spectral triple $\left(\mathcal{B}, \mathscr{H}_{\Sigma}, D_{e q}\right)$, which is proved in Proposition 4.17. Proposition 4.17 also implies that the spectral triple has simple dimension spectrum which is a subset of $\{1,2, \ldots, 2 \ell+1\}$. The fact that every point in $\{1,2, \ldots, 2 \ell+1\}$ is in the dimension spectrum follows from Lemma 4.12. This completes the proof.

4.6. The operators $\boldsymbol{Z}_{\boldsymbol{j}, \boldsymbol{q}}$. We will give a proof of Proposition 4.24 in this section. The main idea is to exploit the isomorphism between the Hilbert spaces $L_{2}\left(S_{q}^{2 \ell+1}\right)$ and $\mathscr{H}_{\Sigma}$ and a detailed analysis of the operators $Z_{j, q}$ to show that certain parts of these operators can be ignored for the purpose of establishing regularity and computing dimension spectrum. Deciding and establishing which parts of these operators can be ignored is the key step here. It should be noted here that a similar analysis has been done by D'Andrea in [9], where $L_{2}\left(S_{q}^{2 \ell+1}\right)$ is embedded in a bigger Hilbert space and certain approximations for the operators $Z_{j, q}$ are proved. But the approximation there is not strong enough to enable the computation of dimension spectrum. Here we prove stronger versions of those approximations, which make it possible to use them to compute the dimension spectrum dealt with in the previous subsection.

We start with a few simple lemmas that will be used repeatedly during the computations in this section.

Lemma 4.26. Let $A \subseteq B \subseteq \Sigma$. Then one has $\mathrm{OP}_{D_{B}}^{-\infty} \otimes \mathcal{E}_{\Sigma \backslash B}^{\infty} \subseteq \mathrm{OP}_{D_{A}}^{-\infty} \otimes \mathcal{E}_{\Sigma \backslash A}^{\infty}$.

Proof. Since

$$
\mathrm{OP}_{D_{B}}^{-\infty}=S\left(\mathscr{H}_{B}\right)=S\left(\mathscr{H}_{A}\right) \otimes S\left(\mathscr{H}_{B \backslash A}\right)=\mathrm{OP}_{D_{A}}^{-\infty} \otimes S\left(\mathscr{H}_{B \backslash A}\right)
$$

and $S\left(\mathscr{H}_{B \backslash A}\right) \subseteq \mathcal{E}_{B \backslash A}^{\infty}$, we have the required inclusion.

Let $A \subseteq \Sigma$. Let $\mathcal{P}$ be a polynomial in $|A|$ variables and let $T$ be the operator on $\mathscr{H}_{A}$ given by

$$
T e_{\gamma}=\mathcal{P}\left(\left\{\gamma_{i}, i \in A\right\}\right) q^{\left|\gamma_{A}\right|} e_{\gamma} .
$$

Since the function $\gamma \mapsto \mathcal{P}\left(\left\{\gamma_{i}, i \in A\right\}\right) q^{\left|\gamma_{A}\right|}$ is a rapid decay function on $\Gamma_{A}$, it follows that $T \in \mathrm{OP}_{D_{A}}^{-\infty}$. 
Lemma 4.27. Let $A \subseteq \Sigma$. Let $T$ and $T_{0}$ be the following operators on $\mathscr{H}_{A}$ :

$$
T e_{\gamma}=q^{\phi\left(\gamma_{A}\right)} Q\left(\psi\left(\gamma_{A}\right)\right) e_{\gamma}, \quad T_{0} e_{\gamma}=q^{\phi\left(\gamma_{A}\right)} e_{\gamma},
$$

where $\phi$ and $\psi$ are some nonnegative functions. If $\phi\left(\gamma_{A}\right)+\psi\left(\gamma_{A}\right)>\left|\gamma_{A}\right|$, then $T-T_{0} \in \mathrm{OP}_{D_{A}}^{-\infty}$.

Proof. This is a consequence of the inequality $\left|1-(1-x)^{\frac{1}{2}}\right|<x$ for $0 \leq x \leq 1$.

Lemma 4.28. Let $A \subseteq \Sigma$. Let $T$ and $T_{0}$ be operators on $\mathscr{H}_{A}$ given by

$$
T e_{\gamma}=q^{\phi\left(\gamma_{A}\right)} Q\left(\psi\left(\gamma_{A}\right)\right)^{-1} e_{\gamma}, \quad T_{0} e_{\gamma}=q^{\phi\left(\gamma_{A}\right)} e_{\gamma}
$$

for some nonnegative functions $\phi$ and $\psi$. If $\phi\left(\gamma_{A}\right)+\psi\left(\gamma_{A}\right)>\left|\gamma_{A}\right|$, then $T-T_{0} \in$ $\mathrm{OP}_{D_{A}}^{-\infty}$.

Proof. For $0<r<1$, one has

$$
\left|1-(1-x)^{-\frac{1}{2}}\right|<c x \quad \text { for } 0 \leq x \leq r,
$$

where $c$ is some fixed constant that depends on $r$. Using this, it follows that the map $\gamma \mapsto q^{\phi(\gamma)}\left|1-\left(1-q^{2 \psi(\gamma)}\right)^{-\frac{1}{2}}\right|$ is a rapid decay function on $\Gamma_{A}$.

For $j \in \Sigma$, we will denote by $\mathcal{E}_{j}$ and $\mathcal{E}$ the $\mathrm{C}^{*}$-algebra $\mathcal{T}$ if $j \neq \ell+1$ and $j=\ell+1$, respectively. Thus $\mathcal{E}_{j}^{\infty}$ will be $\mathcal{T}^{\infty}$ for $j \neq \ell+1$ and $\mathcal{E}^{\infty}=\mathcal{B}$ for $j=\ell+1$. Thus $\mathcal{E}_{\Sigma}^{\infty}$ will stand for the space $\mathcal{T}_{\ell}^{\infty} \otimes \mathcal{E}^{\infty} \otimes \mathcal{T}_{\ell}^{\infty}$. Note that for any subset $A$ of $\Sigma$, one has $\mathrm{OP}_{D_{A}}^{-\infty} \subseteq \mathcal{E}_{\Sigma}^{\infty}$.

Lemma 4.29. Let $A \subseteq \Sigma, a, b, m, n \in \mathbb{N}$ and $n>0$. Let $T_{1}$ and $T_{2}$ be the operators on $\mathscr{H}_{\Sigma}$ given by

$$
\begin{aligned}
& T_{1} e_{\gamma}=Q\left(\left|\gamma_{A}\right|+a\left(\gamma_{\ell+1}\right)_{+}+b\left(\gamma_{\ell+1}\right)_{-}+m\right) e_{\gamma}, \\
& T_{2} e_{\gamma}=Q\left(\left|\gamma_{A}\right|+a\left(\gamma_{\ell+1}\right)_{+}+b\left(\gamma_{\ell+1}\right)_{-}+n\right)^{-1} e_{\gamma} .
\end{aligned}
$$

Then $T_{1}$ and $T_{2}$ are in $\mathcal{E}_{\Sigma}^{\infty}$.

Proof. First note that if $T_{1}^{\prime}$ and $T_{1}^{\prime \prime}$ are operators given by

$$
T_{1}^{\prime} e_{\gamma}=Q\left(\left|\gamma_{A}\right|+a\left|\gamma_{\ell+1}\right|+m\right) e_{\gamma}, \quad T_{1}^{\prime \prime} e_{\gamma}=Q\left(\left|\gamma_{A}\right|+b\left|\gamma_{\ell+1}\right|+m\right) e_{\gamma},
$$

then $T_{1}=P_{\Sigma} T_{1}^{\prime}+\left(I-P_{\Sigma}\right) T_{1}^{\prime \prime}$, where $P_{\Sigma}=\frac{I+F_{\Sigma}}{2}$. By the two previous lemmas, $I-T_{1}^{\prime}$ and $I-T_{2}^{\prime}$ are in $\mathrm{OP}_{D_{B}}^{-\infty}$, where $B=A \cup\{\ell+1\}$. Since $\mathrm{OP}_{D_{B}}^{-\infty}$ is contained in $\mathcal{E}_{\Sigma}^{\infty}$, it follows that $T_{1}^{\prime}, T_{2}^{\prime} \in \mathcal{E}_{\Sigma}^{\infty}$. Since $P_{\Sigma} \in \mathcal{E}_{\Sigma}^{\infty}$, we get $T_{1} \in \mathcal{E}_{\Sigma}^{\infty}$.

For $T_{2}$ the proof is similar. 
We next proceed with a detailed analysis of the operators $Z_{j, q}$. First recall that

$$
U^{*} e_{\gamma}=\xi_{\gamma}^{\prime}=(-1)^{\sum_{i=1}^{\ell}(i-1) \gamma_{i}+\ell\left(\gamma_{\ell+1}\right)_{+}} e_{\boldsymbol{r}^{n, k}, \boldsymbol{s}},
$$

where $s$ is given by

$$
\begin{gathered}
n=\left(\gamma_{\ell+1}\right)_{-}+\sum_{i=\ell+2}^{2 \ell+1} \gamma_{i}, \quad k=\sum_{i=1}^{\ell} \gamma_{i}+\left(\gamma_{\ell+1}\right)_{+}, \\
d_{m}=\sum_{i=1}^{m-1} \gamma_{i}, \quad c_{m}=\sum_{i=1}^{\ell} \gamma_{i}+\left|\gamma_{\ell+1}\right|+\sum_{i=\ell+2}^{2 \ell+2-m} \gamma_{i} \quad \text { for } 1 \leq m \leq \ell . \\
d_{\ell+1}=c_{\ell+1}=\sum_{i=1}^{\ell} \gamma_{i}+\left(\gamma_{\ell+1}\right)_{-} .
\end{gathered}
$$

We will use this correspondence between $e_{\boldsymbol{r}^{n, k}, \boldsymbol{s}}$ and $\xi_{\gamma}^{\prime}$ freely in what follows.

From equation (4.22), we get

$$
\begin{aligned}
\pi\left(u_{1 j}\right) e_{\boldsymbol{r}^{n, k} \boldsymbol{s}}= & \sum_{M \in \mathbb{M}_{j}^{+}} C_{q}\left(1, \boldsymbol{r}^{n, k}, N_{1,1}\right) C_{q}(j, \boldsymbol{s}, M) \kappa\left(\boldsymbol{r}^{n, k}, N_{1,1}\right) e_{\boldsymbol{r}^{n+1, k}, M(\boldsymbol{s})} \\
& +\sum_{M \in \mathbb{M}_{j}^{-}} C_{q}\left(1, \boldsymbol{r}^{n, k}, N_{0,1}\right) C_{q}(j, \boldsymbol{s}, M) \kappa\left(\boldsymbol{r}^{n, k}, N_{0,1}\right) e_{\boldsymbol{r}^{n, k-1}, M(\boldsymbol{s})} .
\end{aligned}
$$

Therefore

$$
\begin{aligned}
Z_{j, q}^{*} e_{\boldsymbol{r}^{n, k}}= & q^{-j+1} \sum_{M \in \mathbb{M}_{j}^{+}} C_{q}\left(1, \boldsymbol{r}^{n, k}, N_{1,1}\right) C_{q}(j, \boldsymbol{s}, M) \kappa\left(\boldsymbol{r}^{n, k}, N_{1,1}\right) e_{\boldsymbol{r}^{n+1, k}, M(s)} \\
& +q^{-j+1} \sum_{M \in \mathbb{M}_{j}^{-}} C_{q}\left(1, \boldsymbol{r}^{n, k}, N_{0,1}\right) C_{q}(j, \boldsymbol{s}, M) \kappa\left(\boldsymbol{r}^{n, k}, N_{0,1}\right) e_{\boldsymbol{r}^{n, k-1}, M(\boldsymbol{s})} .
\end{aligned}
$$

Thus we have $Z_{j, q}^{*}=\sum_{M \in \mathrm{M}_{j}^{+}} S_{M}^{+} T_{M}^{+}+\sum_{M \in \mathbb{M}_{j}^{-}} S_{M}^{-} T_{M}^{-}$, where the operators $S_{M}^{ \pm}$ and $T_{M}^{ \pm}$are given by

$$
\begin{aligned}
& S_{M}^{+} e_{\boldsymbol{r}^{n, k_{\boldsymbol{s}}}}=e_{\boldsymbol{r}^{n+1, k}, M(\boldsymbol{s})}, \quad M \in \mathbb{M}_{j}^{+}, \\
& S_{M}^{-} e_{\boldsymbol{r}^{n, k} k_{\boldsymbol{s}}}=e_{\boldsymbol{r}^{n, k-1}, M(\boldsymbol{s})}, \quad M \in \mathbb{M}_{j}^{-}, \\
& T_{M}^{+} e_{\boldsymbol{r}^{n, k_{s}}}=q^{-j+1} C_{q}\left(1, \boldsymbol{r}^{n, k}, N_{1,1}\right) C_{q}(j, \boldsymbol{s}, M) \kappa\left(\boldsymbol{r}^{n, k}, N_{1,1}\right) e_{\boldsymbol{r}^{n, k_{\boldsymbol{s}}}}, M \in \mathbb{M}_{j}^{+}, \\
& T_{M}^{-} e_{\boldsymbol{r}^{n, k_{\boldsymbol{s}}}}=q^{-j+1} C_{q}\left(1, \boldsymbol{r}^{n, k}, N_{0,1}\right) C_{q}(j, \boldsymbol{s}, M) \kappa\left(\boldsymbol{r}^{n, k}, N_{0,1}\right) e_{\boldsymbol{r}^{n, k_{\boldsymbol{s}}}}, M \in \mathbb{M}_{j}^{-}
\end{aligned}
$$

Lemma 4.30. Let $S_{M}^{ \pm}$be as above. Then $U S_{M}^{ \pm} U^{*} \in \mathcal{E}_{\Sigma}^{\infty}$. 
Proof. Let us first look at the case $M \in \mathbb{M}_{j}^{ \pm}$, where $1 \leq j \leq \ell$. Then one has $S_{M}^{ \pm} \xi_{\gamma}=\xi_{\gamma^{\prime}}$ where $\gamma^{\prime}$ is given by

$$
\gamma_{i}^{\prime}=\left\{\begin{array}{l}
\gamma_{i}+1 \quad \text { if }\left\{\begin{array}{l}
m_{i}=1 \text { and } m_{i+1}=\ell+1-i, \\
m_{2 \ell+2-i}=1 \text { and } m_{2 \ell+3-i}=\ell+2-(2 \ell+3-i),
\end{array}\right. \\
\gamma_{i}-1 \quad \text { if }\left\{\begin{array}{l}
m_{i}=\ell+2-i \text { and } m_{i+1}=1, \\
m_{i}=\ell+2-i \text { and } i=j, \\
m_{2 \ell+2-i}=\ell+2-(2 \ell+2-i) \text { and } m_{2 \ell+3-i}=1,
\end{array}\right. \\
\gamma_{i} \text { otherwise. }
\end{array}\right.
$$

Note that since $1 \leq j \leq \ell$, we have $\gamma_{\ell+1}^{\prime}=\gamma_{\ell+1}$, and $\eta\left(\gamma^{\prime}\right)-\eta(\gamma)$ depends just on $M$ and not on $\gamma$. Therefore $U S_{M}^{ \pm} U^{*}$ is a constant times simple tensor product of shift operators. Thus in this case $U S_{M}^{ \pm} U^{*} \in \mathcal{T}_{\ell}^{\infty} \otimes I \otimes \mathcal{T}_{\ell}^{\infty} \subseteq \mathcal{E}_{\Sigma}^{\infty}$.

Next we look at the case $M \in \mathbb{M}_{\ell+1}^{ \pm}$. Then define $\gamma^{\prime}$ and $\gamma^{\prime \prime}$ as follows:

$$
\begin{gathered}
\gamma_{i}^{\prime}= \begin{cases}\gamma_{i}+1 \quad \text { if }\left\{\begin{array}{l}
m_{i}=1 \text { and } m_{i+1}=\ell+1-i, \\
m_{2 \ell+2-i}=1 \text { and } m_{2 \ell+3-i}=\ell+2-(2 \ell+3-i),
\end{array}\right. \\
\gamma_{i}-1 \quad \text { if }\left\{\begin{array}{l}
m_{i}=\ell+2-i \text { and } m_{i+1}=1, \\
m_{2 \ell+2-i}=\ell+2-(2 \ell+2-i) \text { and } m_{2 \ell+3-i}=1, \\
i=\ell+1,
\end{array}\right. \\
\gamma_{i} \text { otherwise. }\end{cases} \\
\gamma_{i}^{\prime \prime}= \begin{cases}\gamma_{i}+1 & \text { if }\left\{\begin{array}{l}
m_{i}=1 \text { and } m_{i+1}=\ell+1-i, \\
m_{2 \ell+2-i}=1 \text { and } m_{2 \ell+3-i}=\ell+2-(2 \ell+3-i), \\
i=\ell,
\end{array}\right. \\
\gamma_{i}-1 & \text { if }\left\{\begin{array}{l}
m_{i}=\ell+2-i \text { and } m_{i+1}=1, \\
m_{2 \ell+2-i}=\ell+2-(2 \ell+2-i) \text { and } m_{2 \ell+3-i}=1, \\
i=\ell+1,
\end{array}\right. \\
\gamma_{i} \quad \text { otherwise. }\end{cases}
\end{gathered}
$$

Then one has

$$
S_{M}^{ \pm} \xi_{\gamma}= \begin{cases}\xi_{\gamma^{\prime}} & \text { if } \gamma_{\ell+1} \leq 0, \\ \xi_{\gamma^{\prime \prime}} & \text { if } \gamma_{\ell+1}>0 .\end{cases}
$$

Therefore in this case, one has $U S_{M}^{ \pm} U^{*} \in \mathcal{T}_{\ell}^{\infty} \otimes \mathcal{E}^{\infty} \otimes \mathcal{T}_{\ell}^{\infty} \subseteq \mathcal{E}_{\Sigma}^{\infty}$.

We will next take a closer look at the operators $T_{M}^{ \pm}$. For this, we need to compute the quantitites involved in eqs. (4.43) and (4.44) more precisely than we have done earlier. We start with the computation of $\kappa$. From eq. (4.1), we get

$$
\psi\left(\boldsymbol{r}^{n, k}\right)=-\frac{\ell}{2}(n+k+(\ell-1) k)+\frac{\ell(\ell+1)}{2} k=-\frac{\ell}{2}(n-k) .
$$


Therefore

$$
\begin{aligned}
& \psi\left(\boldsymbol{r}^{n, k}\right)-\psi\left(N_{1,1}\left(\boldsymbol{r}^{n, k}\right)\right)=\psi\left(\boldsymbol{r}^{n, k}\right)-\psi\left(\boldsymbol{r}^{n+1, k}\right)=\frac{\ell}{2}, \\
& \psi\left(\boldsymbol{r}^{n, k}\right)-\psi\left(N_{0,1}\left(\boldsymbol{r}^{n, k}\right)\right)=\psi\left(\boldsymbol{r}^{n, k}\right)-\psi\left(\boldsymbol{r}^{n, k-1}\right)=\frac{\ell}{2} .
\end{aligned}
$$

Let us write $\lambda=(n+k, k, \ldots, k, 0)$. We will next compute $d_{\lambda}$, where $d_{\lambda}$ is given by (4.1). One has $d_{\lambda}=\sum_{s} q^{2 \psi(s)}$, where the sum is over all those $s$ for which the top row is $\lambda$. Such an $\boldsymbol{s}$ is of the form (4.25) and one has

$$
\psi(s)=-\frac{1}{2} \ell(n+\ell k)+\frac{1}{2}(\ell-1)(\ell-2) k+\sum_{i=2}^{\ell}\left(c_{i}+d_{i}\right)+d_{\ell+1} .
$$

Thus we have

$$
d_{\lambda}=q^{-\ell(n+k)-2(\ell-1) k} \sum_{\substack{k \leq c_{\ell} \leq c_{\ell-1} \leq \cdots \leq c_{2} \leq n+k \\ 0 \leq d_{2} \leq d_{3} \leq \cdots \leq d_{\ell} \leq k \\ d_{\ell} \leq d_{\ell+1} \leq c_{\ell}}} q^{2\left(\sum_{i=2}^{\ell}\left(c_{i}+d_{i}\right)+d_{\ell+1}\right)}
$$

Now for any $x$, we have

$$
\begin{aligned}
& \sum_{\substack{k \leq c_{\ell} \leq c_{\ell-1} \leq \cdots \leq c_{2} \leq n+k \\
0 \leq d_{2} \leq d_{3} \leq \cdots \leq d_{\ell} \leq k \\
d_{\ell} \leq d_{\ell+1} \leq c_{\ell}}} x^{\left(\sum_{i=2}^{\ell}\left(c_{i}+d_{i}\right)+d_{\ell+1}\right)} \\
& =\left(\sum_{k \leq d_{\ell+1} \leq c_{\ell} \leq c_{\ell-1} \leq \cdots \leq c_{2} \leq n+k} x^{\left(\sum_{i=2}^{\ell} c_{i}+d_{\ell+1}\right)}\right)\left(\sum_{0 \leq d_{2} \leq d_{3} \leq \cdots \leq d_{\ell} \leq k} x^{\left(\sum_{i=2}^{\ell} d_{i}\right)}\right) \\
& +\left(\sum_{k \leq c_{\ell} \leq c_{\ell-1} \leq \cdots \leq c_{2} \leq n+k} x^{\left(\sum_{i=2}^{\ell} c_{i}\right)}\right)\left(\sum_{0 \leq d_{2} \leq d_{3} \leq \cdots \leq d_{\ell} \leq d_{\ell+1}<k} x^{\left(\sum_{i=2}^{\ell} d_{i}+d_{\ell+1}\right)}\right) .
\end{aligned}
$$

If we now use the identity

$$
\sum_{k \leq t_{1} \leq t_{2} \leq \cdots \leq t_{j} \leq n} x^{\left(\sum_{i=1}^{j} t_{i}\right)}=x^{j k} \prod_{i=1}^{j}\left(\frac{1-x^{n-k+i}}{1-x^{i}}\right),
$$

we get

$$
\begin{gathered}
\sum_{\begin{array}{r}
k \leq c_{\ell} \leq c_{\ell-1} \leq \cdots \leq c_{2} \leq n+k \\
0 \leq d_{2} \leq d_{3} \leq \cdots \leq d_{\ell} \leq k \\
d_{\ell} \leq d_{\ell+1} \leq c_{\ell}
\end{array}} x^{\left(\sum_{i=2}^{\ell}\left(c_{i}+d_{i}\right)+d_{\ell+1}\right)} \\
=x^{\ell k} \prod_{i=1}^{\ell}\left(\frac{1-x^{n+i}}{1-x^{i}}\right) \prod_{i=1}^{\ell-1}\left(\frac{1-x^{k+i}}{1-x^{i}}\right) \\
+x^{(\ell-1) k} \prod_{i=1}^{\ell-1}\left(\frac{1-x^{n+i}}{1-x^{i}}\right) \prod_{i=1}^{\ell}\left(\frac{1-x^{k-1+i}}{1-x^{i}}\right)
\end{gathered}
$$




$$
\begin{aligned}
& =x^{(\ell-1) k} \prod_{i=1}^{\ell-1}\left(\frac{1-x^{n+i}}{1-x^{i}}\right) \prod_{i=1}^{\ell-1}\left(\frac{1-x^{k+i}}{1-x^{i}}\right) \frac{1}{1-x^{\ell}}\left(x^{k}\left(1-x^{n+\ell}\right)+1-x^{k}\right) \\
& =x^{(\ell-1) k} \prod_{i=1}^{\ell-1}\left(\frac{1-x^{n+i}}{1-x^{i}}\right) \prod_{i=1}^{\ell-1}\left(\frac{1-x^{k+i}}{1-x^{i}}\right)\left(\frac{1-x^{n+k+\ell}}{1-x^{\ell}}\right) .
\end{aligned}
$$

Thus

$$
d_{\lambda}^{\frac{1}{2}}=q^{-\frac{\ell(n+k)}{2}} \prod_{i=1}^{\ell-1}\left(\frac{Q(n+i)}{Q(i)} \frac{Q(k+i)}{Q(i)}\right) \frac{Q(n+k+\ell)}{Q(\ell)}
$$

Write

$$
\lambda^{\prime}=(n+1+k, k, \ldots, k, 0), \quad \lambda^{\prime \prime}=(n+k-1, k-1, \ldots, k-1,0) .
$$

Then one has

$$
\begin{aligned}
& d_{\lambda}^{\frac{1}{2}} d_{\lambda^{\prime}}^{-\frac{1}{2}}=q^{\ell / 2} \frac{Q(n+1)}{Q(n+\ell)} \frac{Q(n+k+\ell)}{Q(n+k+\ell+1)} \\
& d_{\lambda}^{\frac{1}{2}} d_{\lambda^{\prime \prime}}^{-\frac{1}{2}}=q^{-\ell / 2} \frac{Q(k+\ell-1)}{Q(k)} \frac{Q(n+k+\ell)}{Q(n+k+\ell-1)} .
\end{aligned}
$$

Combining these with (4.45) and (4.46), we get

$$
\begin{aligned}
& \kappa\left(\boldsymbol{r}^{n, k}, N_{1,1}\left(\boldsymbol{r}^{n, k}\right)\right)=q^{\ell} \frac{Q(n+1)}{Q(n+\ell)} \frac{Q(n+k+\ell)}{Q(n+k+\ell+1)}, \\
& \kappa\left(\boldsymbol{r}^{n, k}, N_{0,1}\left(\boldsymbol{r}^{n, k}\right)\right)=\frac{Q(k+\ell-1)}{Q(k)} \frac{Q(n+k+\ell)}{Q(n+k+\ell-1)} .
\end{aligned}
$$

Lemma 4.31. Let $M \in M_{j}^{+}$and $T_{M}^{+}$be as in eq. (4.43). Then $U T_{M}^{+} U^{*} \in$ $\mathrm{OP}_{D_{\ell}}^{-\infty} \otimes \mathcal{T}_{\ell}^{\infty}$ if $j \leq \ell$ or if $j=\ell+1$ and $M \neq N_{\ell}$.

Proof. From Lemma 4.5 and eqs. (4.43) and (4.49), we get, for $M=\left(m_{1}, \ldots, m_{j}\right) \in$ $\mathrm{M}_{j}^{+}$,

$$
\begin{aligned}
T_{M}^{+} e_{\boldsymbol{r}^{n, k} \boldsymbol{s}}= & \operatorname{sgn}(M) q^{\ell-j+1+C\left(\boldsymbol{r}^{n, k}, N_{1,1}\right)+B\left(N_{1,1}\right)+C(\boldsymbol{s}, M)+B(M)} \\
& \cdot \frac{Q(n+1)}{Q(n+\ell)} \frac{Q(n+k+\ell)}{Q(n+k+\ell+1)} \\
& \cdot L^{\prime}\left(\boldsymbol{r}^{n, k}, 1,1\right)\left(\prod_{a=1}^{j-1} L\left(\boldsymbol{s}, a, m_{a}, m_{a+1}\right)\right) L^{\prime}\left(\boldsymbol{s}, j, m_{j}\right) e_{\boldsymbol{r}^{n, k}} .
\end{aligned}
$$

Since $C\left(\boldsymbol{r}^{n, k}, N_{1,1}\right)=k$ and $B\left(N_{1,1}\right)=0$, we get

$$
T_{M}^{+} e_{\boldsymbol{r}^{n, k_{\boldsymbol{s}}}}=\operatorname{sgn}(M) q^{\ell-j+1+B(M)+k+C(\boldsymbol{s}, M)} \phi(\boldsymbol{s}, M) e_{\boldsymbol{r}^{n, k_{\boldsymbol{s}}}},
$$


with $\phi(s, M)$ a product of terms of the form $Q(\psi(\gamma))^{ \pm 1}$ where $\psi(\gamma)=\left|\gamma_{A}\right|+$ $c\left(\gamma_{\ell+1}\right)_{ \pm}+m$ for some subset $A \subseteq \Sigma, c \in\{0,1\}$ and some integer $m$ that does not depend on $s$. Therefore

$$
U T_{M}^{+} U^{*} e_{\gamma}=\operatorname{sgn}(M) q^{\ell-j+1+B(M)+k+C(s, M)} \phi(s, M) e_{\gamma},
$$

where $k$ and $s$ are given by eqs. (4.38)-(4.40). Since $\phi(s, M)$ a product of terms of the form $Q(\psi(\gamma))^{ \pm 1}$, it follows from Lemma 4.29 that the operator $e_{\gamma} \mapsto \phi(s, M) e_{\gamma}$ is in $\mathcal{E}_{\Sigma}^{\infty}$. Next look at the operator $e_{\gamma} \mapsto q^{k+C(s, M)} e_{\gamma}$. Assume that there is some $i \leq j$ such that $m_{i} \neq 1$. Let $p=\min \left\{2 \leq i \leq j: m_{i} \neq 1\right\}$. Then $C(s, M) \geq H_{p-1,1}(s) \geq\left(\gamma_{\ell+1}\right)_{-}$. Therefore

$$
k+C(s, M) \geq k+\left(\gamma_{\ell+1}\right)_{-}=\sum_{i=1}^{\ell} \gamma_{i}+\left|\gamma_{\ell+1}\right| .
$$

Hence $U T_{M}^{+} U^{*} \in \mathrm{OP}_{D_{\ell}}^{-\infty} \otimes \mathcal{T}_{\ell}^{\infty}$. Next assume that $j \leq \ell$ and $m_{i}=1$ for all $i \leq j$. In this case, $C(s, M) \geq H_{j, 1}(s) \geq\left(\gamma_{\ell+1}\right)_{-}$. Therefore again we have

$$
k+C(s, M) \geq k+\left(\gamma_{\ell+1}\right)_{-}=\sum_{i=1}^{\ell} \gamma_{i}+\left|\gamma_{\ell+1}\right|
$$

and hence $U T_{M}^{+} U^{*} \in \mathrm{OP}_{D_{\ell}}^{-\infty} \otimes \mathcal{T}_{\ell}^{\infty}$. Combining the two cases, we have the required result.

Lemma 4.32. Let $M \in \mathrm{M}_{j}^{-}$and $T_{M}^{-}$be as in eq. (4.44). Then $U T_{M}^{-} U^{*} \in \mathrm{OP}_{D_{\ell}}^{-\infty} \otimes \mathcal{T}_{\ell}^{\infty}$ if $M \neq N_{0, j}$.

Proof. From Lemma 4.5 and eqs. (4.44) and (4.50), we get, for $M=\left(m_{1}, \ldots, m_{j}\right) \in$ $\mathrm{M}_{j}^{-}$

$$
\begin{aligned}
T_{M}^{-} e_{\boldsymbol{r}^{n, k} \boldsymbol{s}}=\operatorname{sgn}(M) q^{-j+1+C\left(\boldsymbol{r}^{n, k}, N_{0,1}\right)+B\left(N_{0,1}\right)+C(\boldsymbol{s}, M)+B(M)} & \\
& \cdot \frac{Q(k+\ell-1)}{Q(k)} \frac{Q(n+k+\ell)}{Q(n+k+\ell-1)} L^{\prime}\left(\boldsymbol{r}^{n, k}, 1, \ell+1\right) \\
& \cdot\left(\prod_{a=1}^{j-1} L\left(\boldsymbol{s}, a, m_{a}, m_{a+1}\right)\right) L^{\prime}\left(\boldsymbol{s}, j, m_{j}\right) e_{\boldsymbol{r}^{n, k_{\boldsymbol{s}}}} .
\end{aligned}
$$

Since $C\left(\boldsymbol{r}^{n, k}, N_{0,1}\right)=0$ and $B\left(N_{0,1}\right)=0$, we get

$$
T_{M}^{-} e_{\boldsymbol{r}^{n, k_{s}}}=\operatorname{sgn}(M) q^{-j+1+C(\boldsymbol{s}, M)+B(M)} \phi(\boldsymbol{s}, M) e_{\boldsymbol{r}^{n, k_{s}}},
$$

with $\phi(s, M)$ a product of terms of the form $Q(\psi(\gamma))^{ \pm 1}$ where $\psi(\gamma)=\left|\gamma_{A}\right|+$ $c\left(\gamma_{\ell+1}\right)_{ \pm}+m$ for some subset $A \subseteq \Sigma, c \in\{0,1\}$ and some integer $m$ that does not depend on $s$. Therefore

$$
U T_{M}^{-} U^{*} e_{\gamma}=\operatorname{sgn}(M) q^{-j+1+C(s, M)+B(M)} \phi(s, M) e_{\gamma},
$$


where $k$ and $s$ are given by eqs. (4.38)-(4.40). As in the proof of Lemma 4.31, it is now enough to shoe that $C(\boldsymbol{s}, M) \geq \sum_{i=1}^{\ell} \gamma_{i}+\left|\gamma_{\ell+1}\right|$. Now assume that $m_{i}=1$ for some $i \leq \ell$. Let $p=\min \left\{2 \leq i \leq j: m_{i}=1\right\}$. Then $p \leq \ell$. We then have

$$
\begin{aligned}
C(s, M) & \geq \sum_{i=1}^{p-2} H_{i, \ell+1-i}(s)+H_{p-1,1}(s)+H_{p-1, \ell+2-p}(s)+V_{p-1, \ell+2-p}(s) \\
& \geq \sum_{i=1}^{p-2} \gamma_{i}+\left(\gamma_{\ell+1}\right)_{-}+\gamma_{p-1}+\left(\sum_{i=1}^{\ell} \gamma_{i}+\left(\gamma_{\ell+1}\right)_{+}-\sum_{i=1}^{p-1} \gamma_{i}\right) \\
& =\sum_{i=1}^{\ell} \gamma_{i}+\left|\gamma_{\ell+1}\right| .
\end{aligned}
$$

The result follows.

Remark 4.33. As mentioned in the beginning of this subsection, weaker versions of the two lemmas above have been proved by D'Andrea in [9]. In our notation, he proves that the part of $Z_{j, q}$ be ignored is of the order $q^{k}=q^{\sum_{i=1}^{\ell} \gamma_{i}+\left(\gamma_{\ell+1}\right)_{+}}$, whereas we prove here that one can actually ignore terms of a slightly higher order, namely $q^{\sum_{i=1}^{\ell} \gamma_{i}+\left|\gamma_{\ell+1}\right|}$, which makes it possible to compute $Z_{j, q}$ modulo the ideal $\mathrm{OP}_{D_{\ell}}^{-\infty} \otimes \mathcal{T}_{\ell}^{\infty}$.

Lemma 4.34. Define operators $X_{j}$ on $L_{2}\left(S_{q}^{2 \ell+1}\right)$ by

$$
e_{\boldsymbol{r}^{n, k}, \boldsymbol{s}} \mapsto \begin{cases}(-1)^{j-1} q^{d_{j}} Q\left(d_{j+1}-d_{j}\right) e_{\boldsymbol{r}^{n, k-1}, N_{0, j}(s)} & \text { if } 1 \leq j \leq \ell-1, \\ (-1)^{\ell-1} q^{d_{\ell}} Q\left(d_{\ell+1}-d_{\ell}\right) Q\left(k-d_{\ell}\right) e_{\boldsymbol{r}^{n, k-1}, N_{0, \ell}(s)} & \text { if } j=\ell .\end{cases}
$$

Then one has

$$
U Z_{j, q}^{*} U^{*}-U X_{j} U^{*} \in \mathrm{OP}_{D_{\ell}}^{-\infty} \otimes \mathcal{T}_{\ell}^{\infty}
$$

Proof. In view of the two forgoing lemmas, it is enough to show that

$$
U S_{N_{0, j}}^{-} T_{N_{0, j}}^{-} U^{*}-U X_{j} U^{*} \in \mathrm{OP}_{D_{\ell}}^{-\infty} \otimes \mathcal{T}_{\ell}^{\infty} \quad \text { for } 1 \leq j \leq \ell .
$$

Let us first look at the case $1 \leq j \leq \ell-1$. Observe that

$$
\begin{aligned}
\operatorname{sgn}\left(N_{0, j}\right) & =(-1)^{j-1}, \\
C\left(\boldsymbol{r}^{n, k}, N_{0,1}\right) & =0=B\left(N_{0,1}\right), \\
C\left(\boldsymbol{s}, N_{0, j}\right) & =d_{j}, \\
B\left(N_{0, j}\right) & =j-1 .
\end{aligned}
$$


Therefore from (4.52), we get

$$
\begin{aligned}
U T_{N_{0, j}}^{-} U^{*} e_{\gamma}= & (-1)^{j-1} q^{d_{j}} \frac{Q(k+\ell-1)}{Q(k)} \frac{Q(n+k+\ell)}{Q(n+k+\ell-1)} L^{\prime}\left(\boldsymbol{r}^{n, k}, 1, \ell+1\right) \\
& \cdot\left(\prod_{a=1}^{j-1} L(s, a, \ell+2-a, \ell+1-a)\right) L^{\prime}(s, j, \ell+2-j) e_{\gamma} .
\end{aligned}
$$

From (4.15), one gets

$$
\begin{aligned}
& L^{\prime}\left(\boldsymbol{r}^{n, k}, 1, \ell+1\right) \\
& \quad=\left(\prod_{i=2}^{\ell} \frac{Q(|k-0-i+\ell+1-1|)}{Q(|k-0-i+\ell+1|)}\right) \frac{Q(|k-0-1+\ell+1-1|)}{Q(|n+k-0-1+\ell+1|)} \\
& \quad=\left(\prod_{i=2}^{\ell} \frac{Q(k+\ell-i)}{Q(k+\ell-i+1)}\right) \frac{Q(k+\ell-1)}{Q(n+k+\ell)}=\frac{Q(k)}{Q(n+k+\ell)} .
\end{aligned}
$$

Similarly, from (4.12) one gets, for $1 \leq a \leq \ell-1$,

$$
\begin{aligned}
& L(s, a, \ell+2-a, \ell+1-a) \\
& =\prod_{i=1}^{\ell+1-a} \frac{Q\left(\left|s_{a, i}-s_{a+1, \ell+1-a}-i+\ell+1-a\right|\right)}{Q\left(\left|s_{a, i}-s_{a, \ell+2-a}-i+\ell+2-a\right|\right)} \\
& \cdot \prod_{i=1}^{\ell-a} \frac{Q\left(\left|s_{a+1, i}-s_{a, \ell+2-a}-i+\ell+2-a-1\right|\right)}{Q\left(\left|s_{a+1, i}-s_{a+1, \ell+1-a}-i+\ell+1-a-1\right|\right)} \\
& =\frac{Q\left(c_{a}-d_{a+1}+\ell-a\right)}{Q\left(c_{a}-d_{a}+\ell+1-a\right)} \frac{Q\left(c_{a+1}-d_{a}+\ell-a\right)}{Q\left(c_{a+1}-d_{a+1}+\ell-a-1\right)} \\
& \cdot \prod_{i=2}^{\ell+1-a} \frac{Q\left(k-d_{a+1}-i+\ell+1-a\right)}{Q\left(k-d_{a}-i+\ell+2-a\right)} \prod_{i=2}^{\ell-a} \frac{Q\left(k-d_{a}-i+\ell+1-a\right)}{Q\left(k-d_{a+1}-i+\ell-a\right)} \\
& =\frac{Q\left(c_{a}-d_{a+1}+\ell-a\right)}{Q\left(c_{a}-d_{a}+\ell+1-a\right)} \frac{Q\left(c_{a+1}-d_{a}+\ell-a\right)}{Q\left(c_{a+1}-d_{a+1}+\ell-a-1\right)} \\
& \cdot \prod_{i=1}^{\ell-a} \frac{Q\left(k-d_{a+1}-i+\ell-a\right)}{Q\left(k-d_{a}-i+\ell+1-a\right)} \prod_{i=2}^{\ell-a} \frac{Q\left(k-d_{a}-i+\ell+1-a\right)}{Q\left(k-d_{a+1}-i+\ell-a\right)} \\
& =\frac{Q\left(c_{a}-d_{a+1}+\ell-a\right)}{Q\left(c_{a}-d_{a}+\ell+1-a\right)} \frac{Q\left(c_{a+1}-d_{a}+\ell-a\right)}{Q\left(c_{a+1}-d_{a+1}+\ell-a-1\right)} \\
& \frac{Q\left(k-d_{a+1}+\ell-a-1\right)}{Q\left(k-d_{a}+\ell-a\right)},
\end{aligned}
$$


and from (4.15), for $j \leq \ell-1$,

$$
\begin{aligned}
L^{\prime}(s, j, \ell+2-j) \\
\quad=\prod_{i=1}^{\ell+1-j} \frac{Q\left(\left|s_{j+1, i}-s_{j, \ell+2-j}-i+\ell+2-j-1\right|\right)}{Q\left(\left|s_{j, i}-s_{j, \ell+2-j}-i+\ell+2-j\right|\right)} \\
\quad=\frac{Q\left(c_{j+1}-d_{j}+\ell-j\right)}{Q\left(c_{j}-d_{j}+\ell+1-j\right)}\left(\prod_{i=2}^{\ell-j} \frac{Q\left(k-d_{j}+\ell+1-j-i\right)}{Q\left(k-d_{j}+\ell+2-j-i\right)}\right) \frac{Q\left(d_{j+1}-d_{j}\right)}{Q\left(k-d_{j}+1\right)} \\
\quad=\frac{Q\left(c_{j+1}-d_{j}+\ell-j\right)}{Q\left(c_{j}-d_{j}+\ell+1-j\right)} \frac{Q\left(d_{j+1}-d_{j}\right)}{Q\left(k-d_{j}+\ell-j\right)} .
\end{aligned}
$$

From the above two equations, we get

$$
\begin{aligned}
\left(\prod_{a=1}^{j-1} L(s, a, \ell+2-a, \ell+1-a)\right) L^{\prime}(s, j, \ell+2-j) \\
=\frac{Q\left(d_{j+1}-d_{j}\right)}{Q(k+\ell-1)}\left(\prod_{a=1}^{j-1} \frac{Q\left(c_{a}-d_{a+1}+\ell-a\right)}{Q\left(c_{a+1}-d_{a+1}+\ell-a-1\right)}\right) \\
\cdot\left(\prod_{a=1}^{j} \frac{Q\left(c_{a+1}-d_{a}+\ell-a\right)}{Q\left(c_{a}-d_{a}+\ell+1-a\right)}\right) .
\end{aligned}
$$

Now substituting all these in eq. (4.55), we get

$$
\begin{aligned}
U T_{N_{0, j}}^{-} U^{*} e_{\gamma}=(-1)^{j-1} q^{d_{j}} \frac{Q\left(d_{j+1}-d_{j}\right)}{Q(n+k+\ell-1)} \\
\cdot\left(\prod_{a=1}^{j-1} \frac{Q\left(c_{a}-d_{a+1}+\ell-a\right)}{Q\left(c_{a+1}-d_{a+1}+\ell-a-1\right)}\right) \\
\cdot\left(\prod_{a=1}^{j} \frac{Q\left(c_{a+1}-d_{a}+\ell-a\right)}{Q\left(c_{a}-d_{a}+\ell+1-a\right)}\right) e_{\gamma} .
\end{aligned}
$$

Now note that for $1 \leq a \leq j-1$,

$$
\begin{aligned}
d_{j}+c_{a}-d_{a+1}+\ell-a & \geq \sum_{i=1}^{\ell} \gamma_{i}+\left|\gamma_{\ell+1}\right|, \\
d_{j}+c_{a+1}-d_{a+1}+\ell-a-1 & \geq \sum_{i=1}^{\ell} \gamma_{i}+\left|\gamma_{\ell+1}\right|,
\end{aligned}
$$


and, for $1 \leq a \leq j$,

$$
\begin{gathered}
d_{j}+c_{a+1}-d_{a}+\ell-a \geq \sum_{i=1}^{\ell} \gamma_{i}+\left|\gamma_{\ell+1}\right|, \\
d_{j}+c_{a}-d_{a}+\ell+1-a \geq \sum_{i=1}^{\ell} \gamma_{i}+\left|\gamma_{\ell+1}\right|,
\end{gathered}
$$

and $d_{j}+n+k+\ell-1 \geq \sum_{i=1}^{\ell} \gamma_{i}+\left|\gamma_{\ell+1}\right|$. Therefore by using Lemmas 4.27 and 4.28, we can write, modulo an operator in $\mathrm{OP}_{D_{\ell}}^{-\infty} \otimes \mathcal{T}_{\ell}^{\infty}$,

$$
U T_{N_{0, j}}^{-} U^{*} e_{\gamma}=(-1)^{j-1} q^{d_{j}} Q\left(d_{j+1}-d_{j}\right) e_{\gamma} .
$$

Using eq. (4.42), we get

$$
U S_{N_{0, j}}^{-} U^{*} e_{\gamma}=(-1)^{j-1} e_{\gamma^{\prime}}
$$

where

$$
\gamma_{i}^{\prime}= \begin{cases}\gamma_{i} & \text { if } i \neq j, \\ \gamma_{i}-1 & \text { if } i=j .\end{cases}
$$

Observe also that

$$
U X_{j} U^{*} e_{\gamma}=q^{d_{j}} Q\left(d_{j+1}-d_{j}\right) e_{\gamma^{\prime}},
$$

where $\gamma^{\prime}$ is as above. Therefore we get (4.54) for $j \leq \ell-1$.

In the case $j=\ell$, one has

$$
L^{\prime}(s, \ell, 2)=\frac{Q\left(\left|s_{\ell+1,1}-s_{\ell, 2}\right|\right)}{Q\left(\left|s_{\ell, 1}-s_{\ell, 2}+1\right|\right)}=\frac{Q\left(d_{\ell+1}-d_{\ell}\right)}{Q\left(c_{\ell}-d_{\ell}+1\right)} .
$$

As a result, it follows that

$$
\begin{aligned}
\left(\prod_{a=1}^{\ell-1} L(s, a, \ell+2-a, \ell+1-a)\right) L^{\prime}(s, \ell, 2) \\
=\frac{Q\left(k-d_{\ell}\right)}{Q(k+\ell-1)}\left(\prod_{a=1}^{\ell-1} \frac{Q\left(c_{a}-d_{a+1}+\ell-a\right)}{Q\left(c_{a+1}-d_{a+1}+\ell-a-1\right)}\right) \\
\quad\left(\prod_{a=1}^{\ell} \frac{Q\left(c_{a+1}-d_{a}+\ell-a\right)}{Q\left(c_{a}-d_{a}+\ell+1-a\right)}\right) .
\end{aligned}
$$


As before, substituting all these in eq. (4.55), one gets

$$
\begin{aligned}
U T_{N_{0, \ell}}^{-} U^{*} e_{\gamma}= & (-1)^{\ell-1} q^{d_{\ell}} \frac{Q\left(k-d_{\ell}\right)}{Q(n+k+\ell-1)} \\
& \left(\prod_{a=1}^{\ell-1} \frac{Q\left(c_{a}-d_{a+1}+\ell-a\right)}{Q\left(c_{a+1}-d_{a+1}+\ell-a-1\right)}\right) \\
& \cdot\left(\prod_{a=1}^{\ell} \frac{Q\left(c_{a+1}-d_{a}+\ell-a\right)}{Q\left(c_{a}-d_{a}+\ell+1-a\right)}\right) e_{\gamma} .
\end{aligned}
$$

Application of Lemmas 4.27 and 4.28, now enables us to write the following equality modulo an operator in $\mathrm{OP}_{D_{\ell}}^{-\infty} \otimes \mathcal{T}_{\ell}^{\infty}$ :

$$
U T_{N_{0, \ell}}^{-} U^{*} e_{\gamma}=(-1)^{\ell-1} q^{d_{\ell}} Q\left(k-d_{\ell}\right) Q\left(d_{\ell+1}-d_{\ell}\right) e_{\gamma} .
$$

Using eq. (4.42), we get

$$
U S_{N_{0, \ell}}^{-} U^{*} e_{\gamma}=(-1)^{\ell-1} e_{\gamma^{\prime}}
$$

where

$$
\gamma_{i}^{\prime}= \begin{cases}\gamma_{i} & \text { if } i \neq \ell \\ \gamma_{i}-1 & \text { if } i=\ell\end{cases}
$$

Observe also that

$$
U X_{\ell} U^{*} e_{\gamma}=q^{d_{\ell}} Q\left(k-d_{\ell}\right) Q\left(d_{\ell+1}-d_{\ell}\right) e_{\gamma^{\prime}},
$$

where $\gamma^{\prime}$ is as above. Therefore we obtain (4.54) for $j=\ell$.

Lemma 4.35. Let $X_{j}$ be as in Lemma 4.34. Then one has $U X_{j} U^{*}-Y_{j, q}^{*} \otimes I \in$ $\mathrm{OP}_{D_{\ell}}^{-\infty} \otimes \mathcal{T}_{\ell}^{\infty}$ for $1 \leq j \leq \ell$.

Proof. It follows from eqs. (4.37)-(4.40) that for $j \leq \ell-1$, one in fact has $U X_{j} U^{*}-$ $Y_{j, q}^{*} \otimes I=0$. For $j=\ell$, one has

$$
\left(U X_{j} U^{*}-Y_{j, q}^{*} \otimes I\right) e_{\gamma}=\left(q^{\sum_{i=1}^{\ell-1} \gamma_{i}} Q\left(\gamma_{\ell}+\left(\gamma_{\ell+1}\right)_{-}\right) Q\left(\gamma_{\ell}+\left(\gamma_{\ell+1}\right)_{+}\right)\right) e_{\hat{\gamma}},
$$

where $\hat{\gamma}_{i}=\gamma_{i}-1$ if $i=\ell$ and $\hat{\gamma}_{i}=\gamma_{i}$ for all other $i$. Thus

$$
\left|U X_{j} U^{*}-Y_{j, q}^{*} \otimes I\right| \in \mathrm{OP}_{D_{\ell}}^{-\infty} \otimes \mathcal{T}_{\ell}^{\infty}, \quad \operatorname{sgn}\left(U X_{j} U^{*}-Y_{j, q}^{*} \otimes I\right) \in \mathcal{E}_{\Sigma}^{\infty} .
$$

Therefore $U X_{j} U^{*}-Y_{j, q}^{*} \otimes I \in \mathrm{OP}_{D_{\ell}}^{-\infty} \otimes \mathcal{T}_{\ell}^{\infty}$.

From the two lemmas above (Lemmas 4.34 and 4.35), it follows that one has $U Z_{j, q}^{*} U^{*} \in C^{\infty}\left(S_{q}^{2 \ell+1}\right)$ for $1 \leq j \leq \ell$. Thus we now need only to take care of the case $j=\ell+1$. 
Lemma 4.36. $U Z_{\ell+1, q}^{*} U^{*} \in C^{\infty}\left(S_{q}^{2 \ell+1}\right)$.

Proof. Using Lemmas 4.31 and 4.32 , it is enough to show that

$$
U\left(S_{N_{\ell}}^{+} T_{N_{\ell}}^{+}+S_{N_{0, \ell+1}}^{-} T_{N_{0, \ell+1}}^{-}\right) U^{*} \in C^{\infty}\left(S_{q}^{2 \ell+1}\right) .
$$

From (4.51), we get

$$
\begin{aligned}
T_{N_{\ell}}^{+} e_{\boldsymbol{r}^{n, k}}= & q^{k} \frac{Q(n+1)}{Q(n+\ell)} \frac{Q(n+k+\ell)}{Q(n+k+\ell+1)} \\
& \cdot L^{\prime}\left(\boldsymbol{r}^{n, k}, 1,1\right)\left(\prod_{a=1}^{\ell} L(\boldsymbol{s}, a, 1,1)\right) e_{\boldsymbol{r}^{n, k} \boldsymbol{s}} .
\end{aligned}
$$

From (4.12), we get for $1 \leq a \leq \ell-1$,

$$
\begin{aligned}
L(s, a, 1,1) & \prod_{i=2}^{\ell+2-a} \frac{Q\left(\left|s_{a, i}-s_{a+1,1}-i+1\right|\right)}{Q\left(\left|s_{a, i}-s_{a, 1}-i+1\right|\right)} \prod_{i=2}^{\ell+1-a} \frac{Q\left(\left|s_{a+1, i}-s_{a, 1}-i+1-1\right|\right)}{Q\left(\left|s_{a+1, i}-s_{a+1,1}-i+1-1\right|\right)} \\
= & \prod_{i=2}^{\ell+1-a} \frac{Q\left(c_{a+1}-k+i-1\right)}{Q\left(c_{a}-k+i-1\right)} \prod_{i=2}^{\ell-a} \frac{Q\left(c_{a}-k+i\right)}{Q\left(c_{a+1}-k+i\right)} \\
& \cdot \frac{Q\left(c_{a+1}-d_{a}+\ell+1-a\right)}{Q\left(c_{a}-d_{a}+\ell+1-a\right)} \frac{Q\left(c_{a}-d_{a+1}+\ell+1-a\right)}{Q\left(c_{a+1}-d_{a+1}+\ell+1-a\right)} \\
= & \prod_{i=1}^{\ell-a} \frac{Q\left(c_{a+1}-k+i\right)}{Q\left(c_{a}-k+i\right)} \prod_{i=2}^{\ell-a} \frac{Q\left(c_{a}-k+i\right)}{Q\left(c_{a+1}-k+i\right)} \\
& \cdot \frac{Q\left(c_{a+1}-d_{a}+\ell+1-a\right)}{Q\left(c_{a}-d_{a}+\ell+1-a\right)} \frac{Q\left(c_{a}-d_{a+1}+\ell+1-a\right)}{Q\left(c_{a+1}-d_{a+1}+\ell+1-a\right)} \\
= & \frac{Q\left(c_{a+1}-k+1\right)}{Q\left(c_{a}-k+1\right)} \frac{Q\left(c_{a+1}-d_{a}+\ell+1-a\right)}{Q\left(c_{a}-d_{a}+\ell+1-a\right)} \frac{Q\left(c_{a}-d_{a+1}+\ell+1-a\right)}{Q\left(c_{a+1}-d_{a+1}+\ell+1-a\right)},
\end{aligned}
$$

and for $a=\ell$,

$$
L(s, \ell, 1,1)=\frac{Q\left(\left|s_{\ell, 2}-s_{\ell+1,1}-2+1\right|\right)}{Q\left(\left|s_{\ell, 2}-s_{\ell, 1}-2+1\right|\right)}=\frac{Q\left(d_{\ell+1}-d_{\ell}+1\right)}{Q\left(c_{\ell}-d_{\ell}+1\right)} .
$$


Also from (4.15), we have

$$
\begin{aligned}
L^{\prime}\left(\boldsymbol{r}^{n, k}, 1,1\right)= & \left(\frac{\prod_{i=1}^{\ell} Q(|k-n-k-i+1-1|)}{\prod_{i=2}^{\ell} Q(|k-n-k-i+1|)}\right) \\
& \cdot \frac{1}{Q(|0-n-k-\ell-1+1|)} \\
= & \left(\prod_{i=2}^{\ell} \frac{Q(n+i)}{Q(n+i-1)}\right) \frac{Q(n+1)}{Q(n+k+\ell)} \\
= & \frac{Q(n+\ell)}{Q(n+k+\ell)} .
\end{aligned}
$$

Plugging these in eq. (4.61) and using (4.37), we get

$$
\begin{aligned}
U T_{N_{\ell}}^{+} U^{*} e_{\gamma}=q^{k} & \frac{Q(n+1)}{Q(n+\ell)} \frac{Q(n+k+\ell)}{Q(n+k+\ell+1)} \frac{Q(n+\ell)}{Q(n+k+\ell)} \\
& \cdot\left(\prod_{a=1}^{\ell-1} \frac{Q\left(c_{a+1}-k+1\right)}{Q\left(c_{a}-k+1\right)} \frac{Q\left(c_{a+1}-d_{a}+\ell+1-a\right)}{Q\left(c_{a}-d_{a}+\ell+1-a\right)}\right. \\
& \left.\cdot \frac{Q\left(c_{a}-d_{a+1}+\ell+1-a\right)}{Q\left(c_{a+1}-d_{a+1}+\ell+1-a\right)}\right) \frac{Q\left(d_{\ell+1}-d_{\ell}+1\right)}{Q\left(c_{\ell}-d_{\ell}+1\right)} e_{\gamma} .
\end{aligned}
$$

Thus as earlier, modulo an operator in $\mathrm{OP}_{D_{\ell}}^{-\infty} \otimes \mathcal{T}_{\ell}^{\infty}$, we have the equality

$$
U T_{N_{\ell}}^{+} U^{*} e_{\gamma}=q^{k} e_{\gamma}
$$

Next note that $B\left(N_{0}\right)=\ell, C\left(s, N_{0}\right)=d_{\ell+1}$ and $\operatorname{sgn}\left(N_{0}\right)=(-1)^{\ell}$ so that we get from (4.52)

$$
\begin{aligned}
T_{N_{0}}^{-} e_{\boldsymbol{r}^{n, k} \boldsymbol{s}}= & (-1)^{\ell} q^{d_{\ell+1}} \frac{Q(k+\ell-1)}{Q(k)} \frac{Q(n+k+\ell)}{Q(n+k+\ell-1)} \\
& \cdot L^{\prime}\left(\boldsymbol{r}^{n, k}, 1, \ell+1\right)\left(\prod_{a=1}^{\ell} L(\boldsymbol{s}, a, \ell+2-a, \ell+1-a)\right) e_{\boldsymbol{r}^{n, k} \boldsymbol{s}} .
\end{aligned}
$$

Now using (4.37), (4.56), (4.57) and the fact that

$$
L(s, \ell, 2,1)=\frac{Q\left(\left|s_{\ell, 1}-s_{\ell+1,1}-1+\ell+1-\ell\right|\right)}{Q\left(\left|s_{\ell, 1}-s_{\ell, 2}-1+\ell+2-\ell\right|\right)}=\frac{Q\left(c_{\ell}-d_{\ell+1}\right)}{Q\left(c_{\ell}-d_{\ell}+1\right)},
$$


we get

$$
\begin{aligned}
U T_{N_{0}}^{-} U^{*} e_{\gamma}= & (-1)^{\ell} q^{d_{\ell+1}} \frac{Q(k+\ell-1)}{Q(k)} \frac{Q(n+k+\ell)}{Q(n+k+\ell-1)} \frac{Q(k)}{Q(n+k+\ell)} \\
& \cdot\left(\prod_{a=1}^{\ell-1} \frac{Q\left(c_{a}-d_{a+1}+\ell-a\right)}{Q\left(c_{a}-d_{a}+\ell+1-a\right)} \frac{Q\left(c_{a+1}-d_{a}+\ell-a\right)}{Q\left(c_{a+1}-d_{a+1}+\ell-a-1\right)}\right. \\
& \left.\cdot \frac{Q\left(k-d_{a+1}+\ell-a-1\right)}{Q\left(k-d_{a}+\ell-a\right)}\right) \frac{Q\left(c_{\ell}-d_{\ell+1}\right)}{Q\left(c_{\ell}-d_{\ell}+1\right)} e_{\gamma} .
\end{aligned}
$$

Thus modulo $\mathrm{OP}_{D_{\ell}}^{-\infty} \otimes \mathcal{T}_{\ell}^{\infty}$, we have the equality

$$
U T_{N_{0}}^{-} U^{*} e_{\gamma}=(-1)^{\ell} q^{d_{\ell+1}} e_{\gamma}
$$

Define operators $T^{ \pm}$on $L_{2}\left(S_{q}^{2 \ell+1}\right)$ by

$$
T^{+} \xi_{\gamma}=q^{k} \xi_{\gamma}, \quad T^{-} \xi_{\gamma}=(-1)^{\ell} q^{d_{\ell+1}} \xi_{\gamma}
$$

By eqs. (4.62) and (4.64), it is enough to look at the operators $S_{N_{\ell}}^{+} T^{+}+S_{N_{0}}^{-} T^{-}$.

Now observe that

$$
S_{N_{0}}^{-} \xi_{\gamma}=\left\{\begin{array}{ll}
\xi_{\gamma^{\prime}} & \text { if } \gamma_{\ell+1}>0, \\
\xi_{\gamma^{\prime \prime}} & \text { if } \gamma_{\ell+1} \leq 0,
\end{array} \quad S_{N_{\ell}}^{+} \xi_{\gamma}= \begin{cases}\xi_{\gamma^{\prime \prime \prime}} & \text { if } \gamma_{\ell+1}>0 \\
\xi_{\gamma^{\prime}} & \text { if } \gamma_{\ell+1} \leq 0\end{cases}\right.
$$

where

$$
\gamma_{i}^{\prime}=\left\{\begin{array}{ll}
\gamma_{i}-1 & \text { if } i=\ell+1, \\
\gamma_{i} & \text { otherwise }
\end{array} \quad \gamma_{i}^{\prime \prime}= \begin{cases}\gamma_{i}-1 & \text { if } \ell \leq i \leq \ell+2 \\
\gamma_{i} & \text { otherwise }\end{cases}\right.
$$

and

$$
\gamma_{i}^{\prime \prime \prime}= \begin{cases}\gamma_{i}+1 & \text { if } i=\ell \text { or } i=\ell+2 \\ \gamma_{i}-1 & \text { if } i=\ell+1 \\ \gamma_{i} & \text { otherwise }\end{cases}
$$

Therefore

$$
\left(S_{N_{\ell}}^{+} T^{+}+S_{N_{0}}^{-} T^{-}\right) \xi_{\gamma}= \begin{cases}q^{k} \xi_{\gamma^{\prime \prime \prime}}+(-1)^{\ell} q^{d_{\ell+1}} \xi_{\gamma^{\prime}} & \text { if } \gamma_{\ell+1}>0 \\ q^{k} \xi_{\gamma^{\prime}}+(-1)^{\ell} q^{d_{\ell+1}} \xi_{\gamma^{\prime \prime}} & \text { if } \gamma_{\ell+1} \leq 0\end{cases}
$$

So if we now define

$$
T \xi_{\gamma}= \begin{cases}(-1)^{\ell} q^{\sum_{i=1}^{\ell} \gamma_{i}} \xi_{\gamma^{\prime}} & \text { if } \gamma_{\ell+1}>0 \\ q^{\sum_{i=1}^{\ell} \gamma_{i}} \xi_{\gamma^{\prime}} & \text { if } \gamma_{\ell+1} \leq 0\end{cases}
$$


then one gets from the above equation that $U\left(S_{N_{\ell}}^{+} T^{+}+S_{N_{0}}^{-} T^{-}-T\right) U^{*}$ is in $\mathrm{OP}_{D_{\ell}}^{-\infty} \otimes \mathcal{T}_{\ell}^{\infty}$. Thus it is enough to show that $U T U^{*} \in C^{\infty}\left(S_{q}^{2 \ell+1}\right)$. Now note that

$$
\eta(\gamma)-\eta\left(\gamma^{\prime}\right)= \begin{cases}\ell & \text { if } \gamma_{\ell+1}>0, \\ 0 & \text { if } \gamma_{\ell+1} \leq 0 .\end{cases}
$$

Therefore it follows that $U T U^{*} e_{\gamma}=q^{\sum_{i=1}^{\ell} \gamma_{i}} e_{\gamma^{\prime}}$, i.e., $U T U^{*}=Y_{\ell+1, q}^{*} \otimes I$. Thus we get the required result.

Putting together Lemmas 4.34, 4.35 and 4.36, we get Proposition 4.24.

Acknowledgement. We would like to thank Partha Sarathi Chakraborty for making us work on the problem. In addition, S. Sundar would like to thank his advisor Partha Sarathi Chakraborty for the constant support. He would also like to thank ISI Delhi Centre for its hospitality during his stay there.

\section{References}

[1] S. Ališauskas and Y. F. Smirnov, Multiplicity-free $\mathrm{u}_{q}(n)$ coupling coefficients. J. Phys. A 27 (1994), 5925-5939. Zbl 0834.17018 MR 1304220

[2] P. S. Chakraborty and A. Pal, Equivariant spectral triples on the quantum SU(2) group. K-Theory 28 (2003), 107-126. Zbl 1028.58005 MR 1995873

[3] P. S. Chakraborty and A. Pal, Torus equivariant spectral triples for odd-dimensional quantum spheres coming from $C^{*}$-extensions. Lett. Math. Phys. 80 (2007), 57-68. Zbl 1114.58005 MR 2314844

[4] P. S. Chakraborty and A. Pal, Characterization of $\mathrm{SU}_{q}(1+1)$-equivariant spectral triples for the odd dimensional quantum spheres. J. Reine Angew. Math. 623 (2008), 25-42. Zbl 1158.58003 MR 2458039

[5] A. Connes, Cyclic cohomology, quantum group symmetries and the local index formula for $\mathrm{SU}_{q}$ (2). J. Inst. Math. Jussieu 3 (2004), 17-68. Zbl 1074.58012 MR 2036597

[6] A. Connes, On the spectral characterization of manifolds. Preprint 2008. arXiv:0810.2088

[7] A. Connes and H. Moscovici, The local index formula in noncommutative geometry. Geom. Funct. Anal. 5 (1995), 174-243. Zbl 0960.46048 MR 1334867

[8] L. Dạbrowski, G. Landi, A. Sitarz, W. van Suijlekom, and J. C. Várilly, The Dirac operator on $\mathrm{SU}_{q}$ (2). Comm. Math. Phys. 259 (2005), 729-759. Zbl 1090.58504 MR 2174423

[9] F. D'Andrea, Noncomutative geometry and quantum group symmetries. Ph.D. Thesis, SISSA, Trieste 2007. arXiv:0811.3187

[10] J. H. Hong and W. Szymański, Quantum spheres and projective spaces as graph algebras. Comm. Math. Phys. 232 (2002), 157-188. Zbl 1015.81029 MR 1942860

[11] A. Klimyk and K. Schmüdgen, Quantum groups and their representations. Texts Monographs Phys., Springer-Verlag, Berlin 1997. Zbl 0891.17010 MR 1492989 
[12] L. B. Schweitzer, A short proof that $M_{n}(A)$ is local if $A$ is local and Fréchet. Internat. $J$. Math. 3 (1992), 581-589. Zbl 0804.46054 MR 1168361

[13] L. B. Schweitzer, Spectral invariance of dense subalgebras of operator algebras. Internat. J. Math. 4 (1993), 289-317. Zbl 0804.46081 MR 1217384

[14] W. van Suijlekom, L. Dạbrowski, G. Landi, A. Sitarz, and J. C. Várilly, The local index formula for $\mathrm{SU}_{q}$ (2). K-Theory 35 (2005), 375-394. Zbl 1098.58007 MR 2240238

[15] S. L. Woronowicz, Tannaka-Kreĭn duality for compact matrix pseudogroups. Twisted SU(N) groups. Invent. Math. 93 (1988), 35-76. Zbl 0664.58044 MR 943923

Received March 2, 2009

A. Pal, Indian Statistical Institute, 7, SJSS Sansanwal Marg, New Delhi-110 016, India

E-mail: arup@isid.ac.in

S. Sundar, Institute of Mathematical Sciences, CIT Campus, Chennai-600 113, India

E-mail: ssundar@imsc.res.in 